\title{
THE CRYOGENIC DARK MATTER SEARCH (CDMS-II) EXPERIMENT - FIRST RESULTS FROM THE SOUDAN MINE
}

\author{
A DISSERTATION \\ SUBMITTED TO THE DEPARTMENT OF PHYSICS \\ AND THE COMMITTEE ON GRADUATE STUDIES \\ OF STANFORD UNIVERSITY \\ IN PARTIAL FULFILLMENT OF THE REQUIREMENTS \\ FOR THE DEGREE OF \\ DOCTOR OF PHILOSOPHY
}

Clarence Leeder Chang

September 2004 
(c) Copyright by Clarence Leeder Chang 2005

All Rights Reserved 
I certify that I have read this dissertation and that, in my opinion, it is fully adequate in scope and quality as a dissertation for the degree of Doctor of Philosophy.

Blas Cabrera
(Principal Adviser)

I certify that I have read this dissertation and that, in my opinion, it is fully adequate in scope and quality as a dissertation for the degree of Doctor of Philosophy.

\section{Michael Peskin}

I certify that I have read this dissertation and that, in my opinion, it is fully adequate in scope and quality as a dissertation for the degree of Doctor of Philosophy.

\section{Doug Osheroff}

Approved for the University Committee on Graduate Studies: 


\section{Abstract}

There is an abundance of evidence that the majority of the mass of the universe is in the form of non-baryonic non-luminous matter that was non-relativistic at the time when matter began to dominate the energy density. Weakly Interacting Massive Particles, or WIMPs, are attractive cold dark matter candidates because they would have a relic abundance today of $\sim 0.1$ which is consistent with precision cosmological measurements. WIMPs are also well motivated theoretically. Many minimal supersymmetric extensions of the Standard Model have WIMPs in the form of the lightest supersymmetric partner, typically taken to be the neutralino.

The CDMS II experiment searches for WIMPs via their elastic scattering off of nuclei. The experiment uses Ge and Si ZIP detectors, operated at $<50 \mathrm{mK}$, which simultaneously measure the ionization and athermal phonons produced by the scattering of an external particle. The dominant background for the experiment comes from electromagnetic interactions taking place very close to the detector surface. Analysis of the phonon signal from these interactions makes it possible to discriminate them from interactions caused by WIMPs. This thesis presents the details of an important aspect of the phonon pulse shape analysis known as the "Lookup Table Correction." The Lookup Table Correction is a position dependent calibration of the ZIP phonon response which improves the rejection of events scattering near the detector surface.

The CDMS collaboration has recently commissioned its experimental installation at the Soudan Mine. This thesis presents an analysis of the data from the first WIMP search at the Soudan Mine. The results of this analysis set the world's lowest exclusion limit making the CDMS II experiment at Soudan the most sensitive WIMP search to this date. 
In loving memory of Helena Hsu. 


\section{Acknowledgements}

It goes without saying that there are a multitude of people without whom this thesis would be impossible. Regretfully, there is only room to mention a few of them.

First, I would like to thank Blas Cabrera. He is an excellent leader, a wise advisor, and an exceptional scientist. Blas is also a man of great patience which is a much needed virtue when one has to deal with my work, or lack thereof. I believe Blas has created the ideal environment for me to grow as a young scientist and is an excellent role model for physicists.

I would also like to thank the entire CDMS collaboration for all of their labor. In particular, Blas Cabrera, Paul Brink, Vuk Mandic, Walter Ogburn, Joel Sander, and Long Duong have made extensive sacrifices to get things to work at Soudan. Without their efforts, CDMS would not be where it is today.

A number of people have contributed significantly to my growth as a scientist. Aaron Miller, Tali Figueroa, and Tarek Saab first introduced me to the world of dilution refrigerators. Rick Gaitskell and Richard Schnee introduced me to data analysis and the mysteries of Dark Pipe.

Dan Bauer, Pat Castle, Astrid Tomada, Betty Young, Mike Hennessy, Jim Perales, and Larry Novak have made working on CDMS at Stanford a joyful experience. And my good friends Hiro, Rhiju, Walter, Tyrone, Lee, and Fong have made my life in the bay area extremely fulfilling.

Lastly, I thank my family. Life is always easier when you know you are loved. 


\section{Contents}

Abstract $\quad$ V

$\begin{array}{ll}\text { Acknowledgements } & \text { ix }\end{array}$

1 Introduction 1

1.1 Hints of Physics Beyond the Standard Model . . . . . . . . . . . . . . 1

1.1.1 The Gauge Hierarchy Problem . . . . . . . . . . . . . . . . . . 1

1.1.2 The Problem of Dark Matter . . . . . . . . . . . . . . 2

1.2 Supersymmetry . . . . . . . . . . . . . . . . . . . . 10

1.2.1 Preliminaries . . . . . . . . . . . . . . . . 10

1.2.2 The MSSM Framework . . . . . . . . . . . . . . . . . . 11

1.2.3 SUSY and the Gauge Hierarchy Problem . . . . . . . . . . . . 12

1.2.4 SUSY and Dark Matter . . . . . . . . . . . . . . . . 13

1.2.5 Accelerator and Cosomological Constraints on MSSMs . . . . 15

1.3 Direct Detection of Dark Matter . . . . . . . . . . . . . . . . 15

1.3.1 Expected Signal . . . . . . . . . . . . . . . . . . 16

1.3.2 Some Current Direct Detection Experiments . . . . . . . . . . 21

2 Detectors $\quad 25$

2.1 The Phonon Measurement . . . . . . . . . . . . . . . 25

2.1.1 Phonons in the Crystal . . . . . . . . . . . . . . 25

2.1.2 Measuring Phonon Energy using QETs . . . . . . . . . . . . . 27

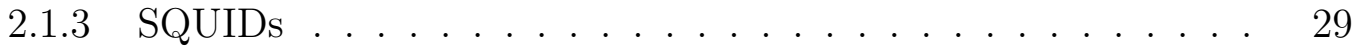

2.1.4 TES Sensitivity to Bias Conditions . . . . . . . . . . . . 31 
2.2 The Ionization Measurement . . . . . . . . . . . . . . . . . . . 33

2.2 .1 Measuring the Ionziation . . . . . . . . . . . . . . 33

2.2.2 Poor Charge Collection . . . . . . . . . . . . . . . . . . 34

2.2 .3 Quenching and Discrimination . . . . . . . . . . . 37

2.3 Event Discrimination . . . . . . . . . . . . . . . . . . . . . . . 37

2.3.1 Non-WIMP nuclear recoils - "Neutrons" . . . . . . . . . . . 39

2.3.2 Bulk Electron Recoils - "Gammas" . . . . . . . . . . . . . . . 39

2.3.3 Surface Electron Recoils - "Betas" . . . . . . . . . . . . . . 42

3 Event Reconstruction $\quad 45$

3.1 Reconstructing the Interaction Location _ . . . . . . . . . . 45

3.1.1 Parameters used for the Reconstruction . . . . . . . . . . 45

3.1.2 The 3-dimensional Position Coordinate . . . . . . . . . . . 46

3.2 Position Dependence of the Detector Response . . . . . . . . . . . 49

3.2.1 Risetime Discrimination and Position Variation . . . . . . . 49

$3.2 .2 T_{c}$ Gradients . . . . . . . . . . . . . . . 53

3.2.3 Intrinsic variation of the phonon signal and degenerate pulse shape analysis . . . . . . . . . . . . . . . . 53

4 The Phonon Lookup Table $\quad 55$

4.1 The Algorithm . . . . . . . . . . . . . . . . . . . 55

4.1 .1 Event Selection . . . . . . . . . . . . . . 55

4.1.2 Linearizing the Energy Response . . . . . . . . . . 56

4.1.3 Averaging over nearest neighbors _... . . . . . . 56

4.1.4 Applying the Correction . . . . . . . . . . . 58

4.2 The Lookup Table in Real Life . . . . . . . . . . . . . . . . . . 59

4.3 The Lookup Table and Soudan R118 . . . . . . . . . . . . . 60

5 The CDMS II Soudan Installation $\quad 65$

5.1 The Soudan Mine . . . . . . . . . . . . . . . . . . . . 65

5.2 Cryogenics . . . . . . . . . . . . . . . . . . 66

5.3 Shielding . . . . . . . . . . . . . . . . . . . . . . . . . 69 
5.4 Electronics and Data Acquisition . . . . . . . . . . . 71

5.5 Online Data Analysis . . . . . . . . . . . . . . . . . . . 73

6 The Deep Site Measurement: Soudan Run $118 \quad 75$

6.1 Run Overview . . . . . . . . . . . . . . . . . . . . . . . . 75

6.2 Energy Calibration . . . . . . . . . . . . . . . . . 77

6.2 .1 Ionization . . . . . . . . . . . . . . . . 77

6.2 .2 Phonons . . . . . . . . . . . . . . . . . 78

6.3 Cut Definitions . . . . . . . . . . . . . . . . . . 82

6.4 Efficiencies . . . . . . . . . . . . . . . . . . . . . . . 85

6.5 Background Estimates . . . . . . . . . . . . . . 86

6.5 .1 Neutrons . . . . . . . . . . . . . . . . . . . . . 87

6.5 .2 Photons . . . . . . . . . . . . . . . . . . 88

6.5.3 Radioactive Betas . . . . . . . . . . . . . . . . . . 88

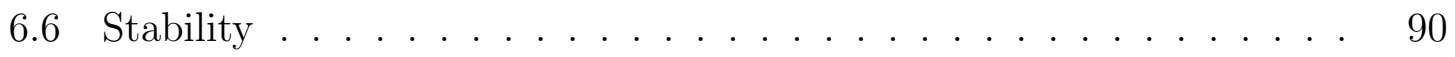

6.6.1 Stability of the Ionizaton and Recoil Calibrations . . . . . . 90

6.6.2 Stability of the Cut Efficiencies . . . . . . . . . . . 95

6.7 Exposure and Expected Sensitivity _. . . . . . . . . . . . . 102

6.7.1 Calculating the Exposure . . . . . . . . . . . . . . . 102

6.7 .2 Calculating the Expected Sensitivity . . . . . . . . . . 102

7 Conclusion and Discussion 107

7.1 Results . . . . . . . . . . . . . . . . . . . 107

7.2 The Next Steps... . . . . . . . . . . . . . . . . . . . . . . . . . . . . . 112

7.2 .1 CDMS II . . . . . . . . . . . . . . . . . . . . . . 112

7.2 .2 Beyond CDMS II . . . . . . . . . . . . . . . . . . . . 113

7.3 Concluding Remarks . . . . . . . . . . . . . . . . . . . . 114

A Instructions and Code for Making Lookup Tables 115

A.1 Instructions . . . . . . . . . . . . . . . . . . . 115

A.2 Code for Selecting Good Events . . . . . . . . . . . . . 116

A.3 Code for Making the Table . . . . . . . . . . . . . . . . . . . 121 
A.4 Code for Some Useful Table Utilities . . . . . . . . . . . . . . . 126

Bibliography

131 


\section{List of Tables}

6.1 Table listing expected leakages for gammas, contamination betas, and neutrons. . . . . . . . . . . . . . . . . . . . 90

6.2 Table summarizing the estimated variation between the background data and the neutron calibration for the ionization yield, qinner, and phonon timing cuts. The values are conservative since they correspond to the maximum deviation between the phonon analysis threshold and $50 \mathrm{keV}$. The total systematic error for the combined Ge ZIPs is less than $7 \%$. . . . . . . . . . . . . . . . . 102

7.1 Table summarizing the results of applying the cuts described in Sec. 6.3 to the Ge detectors. . . . . . . . . . . . . . . . . . . . 107 


\section{List of Figures}

1.1 Fermion loop correction to the Higgs self energy. There are also corrections from loops with the gauge bosons and the Higgs itself. In the Standard Model, the $t, W$, and $h$ loops give the largest contributions.

1.2 Galactic rotation curve for galaxy NGC3198 [4]. The non-Keplerian behavior of the flat rotation curve (instead of $1 / \sqrt{r}$ ) indicates a large fraction of the galactic mass is non-luminous. The dashed line corresponds to the mass distribution of visible matter. The dotted line corresponds to the distribution of gas. The dot-dashed line corresponds to the mass distribution of the non-luminous halo. . . . . . . . . . . .

1.3 Average rotation curves for $\sim 1000$ spiral galaxies. Each plot shows the average rotation curve (solid line) for galaxies with the indicated luminosities (in absolute magnitude). The dotted lines corresponds to the contribution from the visible disc and the dashed lines correspond to the contribution from a dark halo. Figure from [5] . . . . . . . .

1.4 Exclusion limits for combined data from EROS and MACHO [8]. The top figure shows exclusion curves for five models considered by EROS [7] and the bottom figure shows exclusion curves for the eight models considered by MACHO $[6] \ldots \ldots \ldots \ldots \ldots$. . . . . . . . . . .

1.5 Excluded regions in the $\left(\omega_{b}, \omega_{d}\right)$ plane for combined SDSS and WMAP analysis. $\omega_{b}=\Omega_{b} h^{2}$ is the baryonic density and $\omega_{d}=\Omega_{d} h^{2}$ is the nonbaryonic matter density $[11] \ldots \ldots \ldots \ldots$ 
1.6 The number density of a stable particle versus time. When $\left\langle\sigma_{A} v\right\rangle=H$, the particle freezes out and the number density remains constant. The solid line corresponds to the equilibrium density. The dashed lines indicate the relic abundance for particles with different annihilation rates. Particles with larger annihilation rates freeze out later leaving a smaller relic abundance. Figure from $[20] . \ldots \ldots$. . . . . . .

1.7 A boson loop contribution to the Higgs self energy that would exactly cancel the contribution from its fermion superpartner shown in Fig. 1.1. 12

1.8 Exclusion plot of various MSSM theories for parameters in the $\left(m_{0}, m_{1 / 2}\right)$ plane for $\tan \beta=10$ (left) and $\tan \beta=55$ (right). $m_{1,2}$ is the GUT gaugino unification mass, $m_{0}$ is the mass of the pseudoscalar Higgs. For both plots $\mu>0$. Note the light green region indicating the relic abundance constraints $[32] \ldots \ldots \ldots \ldots \ldots \ldots \ldots \ldots \ldots$

1.9 Some of the Feynman diagrams contributing to the spin independent neutralino nucleon cross section. For some models, couplings of the neutralino to gluons through quark and squark loops becomes important. 18

1.10 (a) The Woods-Saxon form factor for Ge, Si, and Xe. (b) The differential WIMP elastic scattering rate for $\mathrm{Ge}, \mathrm{Si}$, and Xe. (c) The integrated WIMP elastic scattering rate for Ge, Si, and Xe. Figure from [37]. . 20

1.11 Plot of $\sigma_{S I}$ per nucleon versus WIMP mass. Exclusion curves for EDELWEISS (black dashed) [40], CDMS I - SUF (blue dashed) [41, 43], CDMS II - SUF (blue solid) [42, 37], ZEPLIN (magenta solid), and CRESST (magenta dashed). Cyan region corresponds to allowed models from Fig. 1.8[32]. Red region is the DAMA detection claim [39]. Limitplot courtesy of $[44] \ldots \ldots \ldots$ 
2.1 Diagram of a ZIP detector. Bottom right is the "Ionization Side" showing a large inner electrode and an outer guard ring. Bottom left is the "Phonon Side" whose surface is divided into four quadrants labeled A, B, C, and D. The axis shown defines the basis for the $\mathrm{x}-\mathrm{y}$ coordinate system. Each quadrant has 148 cells (top left) each of which have 24 QETs. A QET (top right) consists of a $1 \mu$ thick strip of $\mathrm{W}$ connected to $8 \mathrm{Al}$ collector fins. . . . . . . . . . . . . . .

2.2 Band diagram of the Al-W overlap region. Should quasi particles in the overlap lose some of their energy, they will be unable to leave the W since they would be sub-gap in the $\mathrm{Al} \ldots \ldots$. . . . . . . . .

2.3 Schematic of TES current measurement circuit. The Input coil is the coil on the left. The feedback coil is the coil on the right. . . . . . . .

2.4 A saturated (top) and unsaturated (bottom) TES trace showing the change in pulse shape due to TES saturation. In both cases, the total area corresponds to the energy of the pulse. . . . . . . . . . . .

2.5 Noise power spectrum of a biased TES showing the $\mathrm{L} / \mathrm{R}$ roll off at $\sim 160 \mathrm{kHz}$ from the SQUID input coil. . . . . . . . . . . . . . . 33

2.6 Ionization circuit schematic . . . . . . . . . . . . . . . . . 34

2.7 Schematic of the band structure at the $\mathrm{Ge}-\alpha$-Si interface. The $\alpha$-Si creates a small potential barrier for both electrons and holes making it more difficult for charges to drift into the wrong electrode. Mid-gap states that may define the alignment of the gaps are also shown. Figure from [43]. . . . . . . . . . . . . . . . . . . .

2.8 A plot of ionization versus recoil for a gamma $\left({ }^{133} \mathrm{Ba}\right)$ and neutron $\left({ }^{252} \mathrm{Cf}\right)$ calibration. The gamma calibration is red. The neutron calibration is blue. There are two distinct populations corresponding to electron recoils and nuclear recoils. . . . . . . . . . . . . . . . . . 38

2.9 Plot of the ionization yield parameter (ionization/recoil) for a gamma $\left({ }^{133} \mathrm{Ba}\right)$ and neutron $\left({ }^{252} \mathrm{Cf}\right)$ calibration showing the electron $(y \sim 1)$ and nuclear $(y \sim 0.3)$ recoil bands. The gamma calibration is in red and the neutron calibration is in blue. . . . . . . . . . . . . 
2.10 Plot showing fit of the band centroids, $Y_{0}\left(E_{R}\right)$, to the means of the yield distributions for neutrons (diamonds) and gammas (circles). . .

2.11 Plots showing the fits of the widths, $\sigma\left(E_{R}\right)$, to the standard deviation of the yield distributions for gammas (left) and neutrons (right). . . .

2.12 A plot showing the use of phonon pulse shape to discriminate between surface electron recoils with poor charge collection and bulk nuclear recoils. . . . . . . . . . . . . . . . . . . .

3.1 Delay plot (right) of the source configuration shown (left). The sources are a ${ }^{241} \mathrm{Am}$ (large source between channels A and B) and a collimated ${ }^{109} \mathrm{Cd}$ (small dots). The non-circular shape of the source "blobs" indicates the loss of resolution along the radial coordinate. The increased density of points near the edge indicates that the radial coordinate is piling up or even folding back. . . . . . . . . . . . . . . . .

3.2 Box plot for $\mathrm{G} 31$ with the ${ }^{109} \mathrm{Cd}$ from Fig. 3.1. The 2 inner collimator holes in channels $\mathrm{B}$ and $\mathrm{C}$ are highlighted. The definition of the phonon partition coordinates yields a square shape. . . . . . . . . . . . .

3.3 Yield vs recoil plot for events shown in Fig. 3.2. The indicated points from the ${ }^{109} \mathrm{Cd}$ blobs have reduced charge collection. . . . . . . . . . .

3.4 Delay plot (left) and box plot (top right). Events in the outer electrode guard ring are highlighted in red prominently showing that both location parameters are double valued. . . . . . . . . . . . .

3.5 A plot for Z5 gammas of the 2D position manifold in the 3D space of xppart, yppart, and normalized radius for a slice in yppart. Events marked by red circles are towards the edge of the detector because they have substantial energy in the outer electrode. Points off of the manifold marked by green x's are most likely internal multiple scatters. 48 
3.6 Left: Risetime distributions for channel B for all events (solid), y > 0.7 (dashed), and high energy gammas (dotted). Note that low energy gammas with full charge collection $(\mathrm{y}>0.7)$ have a fast risetime tail. Right: Histograms of risetime distributions for channel B (solid) and channel C (dashed). . . . . . . . . . . . . . . . .

3.7 Landscape plot of yield (qsum/recoil) and total phonon risetime for Z1. Each column corresponds to a rotation of the boxplot with the indicated slice as the horizontal coordinate for the plots above the box plots. Z1 has the largest signal variation of all the detectors. It is known to have the largest $T_{c}$ gradient. . . . . . . . . . . . .

3.8 Landscape plot of yield (qsum/recoil) and total phonon risetime for Z1. Each column corresponds to a rotation of the boxplot with the indicated slice as the horizontal coordinate for the plots above the box plots. The extremely small $T_{c}$ gradient of Z5 suggests that the pulse shape variation has a component that is not related to $T_{c}$. . . . . . 52

4.1 left: A generic delay plot with a uniform binning scheme shown by the grid. right: a 75 nearest neighbor binning scheme where 3 clusters of 75 nearest neighbors are shown. Note the variation of the "size" of each cluster for different positions in the delay plot. . . . . . . . . . . 57

4.2 Z1 yield vs position before (top row) and after (middle row) the correction 62

4.3 Z1 total risetime vs position before (top row) and after (middle row) the correction ..................... 63

5.1 Diagram of the CDMS II lab area in the Soudan Underground Laboratory. . . . . . . . . . . . . . . . 66

5.2 A diagram outlining the layout of the connection of the Ice Box (center) to the Oxford cryostat (left). The temperatures of each layer (outermost to innermost) is supposed to be $300 \mathrm{~K}, 77 \mathrm{~K}, 4 \mathrm{~K}, 600 \mathrm{mK}, 50 \mathrm{mK}$, and $20 \mathrm{mK} \ldots \ldots \ldots$. . . . . . . . . . . . . . . . 67

5.3 Diagram of veto and shielding for the CDMS II installation from the side and top . . . . . . . . . . . . . . . . . 70 
5.4 CDMS II Tower 1. Z1 is at the top of the Tower and Z6 is at the bottom 71

6.1 Position dependence (left) of the ionization response due to currently unknown causes. This position dependence can be removed (right) and is negligible at lower energies. . . . . . . . . . . . . . . . . . . . . . 77

6.2 Charge calibration of the 4 Ge ZIPs. Z1 (top left), Z2 (top right), Z3 (bottom left), and Z5 (bottom right). . . . . . . . . . . . . . 79

6.3 10 keVee line in ionization from activation of Ge by the neutron calibrations. . . . . . . . . . . . . . . . . .

6.4 $10 \mathrm{keV}$ line in phonons from activation of Ge by the neutron calibrations. The line for $\mathrm{Z} 1$ in phonons is wider since the correction is worse at low energies. . . . . . . . . . . . . . . . . . .

6.5 Comparison between Monte Carlo and data for Ge (left) and Si (right) detectors. Top plots are ionization spectra for gammas from ${ }^{133} \mathrm{Ba}$. Bottom plots are recoil spectra for neutrons from ${ }^{252} \mathrm{Cf}$. The agreement confirms the phonon and ionization calibrations as well as the claim that the phonon energy measurement is unquenched. . . . . . . . .

6.6 Traces for phonon channel $\mathrm{A}$ in Z1, Z2, and Z3 for an electronics "glitch." Such events are rejected by demanding energy in only one detector. . . . . . . . . . . . . . . . .

6.7 Traces illustrating the two classes of pile up events. The first class (left) have events occuring after the trigger and are rejected based on the $\chi^{2}$ of the ionization pulse. The second class (right) have events occuring before the trigger and are rejected based on the standard deviation of the pre-pulse baseline for the phonon channels. . . . . . . . . . . . 
6.8 Effects of threshold cuts, timing cuts, and nuclear recoil band on neutrons for Z1 (top left), Z2 (top right), Z3 (middle left), Z4 (middle right), Z5 (bottom left), and Z6 (bottom right). Blue dots correspond to single scatter inner electrode events after pile up rejection. Red diamonds are events passing phonon timing cuts. Solid lines indicate the analysis thresholds and the nuclear recoil acceptance band. Compare with Fig. 7.1. . . . . . . . . . . . . . . . . . . . .

6.9 Hardware trigger (solid) and ionization analysis threshold (dashed) efficiencies versus recoil. The hardware trigger efficiency is unity above our phonon analysis thresholds. . . . . . . . . . . . .

6.10 Efficiencies versus recoil for the Pile Up Cut (solid green), Nuclear Recoil Cut (dashed green), Qinner Cut (solid black), and Phonon Timing Cut (dashed black). Note that the Phonon Timing cut reduces the overall efficiency at lower energies while the Qinner cut dominates the efficiency at high energy. The Qinner efficiency drops at high energies due to low statistics and non-uniformity in the illumination of the detector. The efficiency for WIMPs is expected to remain constant making this estimate conservative. The impact of this drop should be marginal since there is very little expected WIMP signal above $50 \mathrm{keV}$.

6.11 Ionization spectra of three $2 \mu \mathrm{Ci}^{133} \mathrm{Ba}$ calibrations: 12/05-12/16 (green), 12/16-12/18 (red), and 1/6 (blue) for the Ge ZIPs. . . . . . . . . . . 92

6.12 Mean and width of events in the Electron Recoil band for four sets of WIMP search data: 10/11-11/1 (green), 11/1-11/20 (red), 11/20-12/15 (blue), and 12/15-1/11 (black). . . . . . . . . . . . . .

6.13 Same as Fig. 6.12 only showing the difference of the means from yield $=1$. 10/11-11/1 (green line), 11/1-11/20 (red diamonds), 11/20-12/15 (blue circles), and 12/15-1/11 (black dots). All of the Ge ZIPs deviated from yield $=1$ by less than $5 \%$. . . . . . . . . . . . .

6.14 Comparison of Electron Recoil band pass fraction between the WIMP search data (black) and the neutron calibrations (red). . . . . . . . . 96 
6.15 Comparison of Qinner cut pass fraction between the WIMP search data (black) and the neutron calibrations (red). . . . . . . . . . .

6.16 Comparison of Phonon Timing cut pass fraction between the WIMP search data (black) and the neutron calibrations (red). . . . . . . . . 98

6.17 Electron Recoil band pass fraction difference between WIMP search data (black) and the neutron calibrations (red). . . . . . . . . . . . 99

6.18 Qinner cut pass fraction difference between WIMP search data (black) and the neutron calibrations $($ red) . . . . . . . . . . . . . . 100

6.19 Phonon Timing cut pass fraction difference between WIMP search data (black) and the neutron calibrations (red). . . . . . . . . . . . . . 101

6.20 Combined efficiencies for the 6 ZIPs. We fit the efficiencies to a cubic. Note that Z1 has an efficiency of zero below its $20 \mathrm{keV}$ analysis threshold while all the other detectors have efficiencies of zero below 5 $\mathrm{keV}$

6.21 Energy dependent exposure in kg days for the summed Ge ZIPs (Z1, Z2, Z3, Z5). The exposure is zero below our analysis threshold of 5 $\mathrm{keV}$. The discontinuity at $20 \mathrm{keV}$ corresponds to the $20 \mathrm{keV}$ analysis threshold for Z1. . . . . . . . . . . . . . . . . . . . . . . . . . 104

6.22 Expected sensitivity. $50 \%$ of the simulated experiments lie in the yellow shaded region. The thick black curve corresponds to the median of the ensemble.

7.1 Plots for Z1 (top left), Z2 (top right), Z3 (middle left), Z4 (middle right), Z5 (bottom left), and Z6 (bottom right) showing the application of the analysis cuts to the WIMP search data. Blue dots correspond to single scatter inner electrode events after pile up rejection. The red diamonds are events passing the phonon timing cuts. Solid lines indicate the analysis thresholds and the nuclear recoil acceptance bands. Compare with Fig. 6.8. Only one candidate event in Z5 at $\sim 60 \mathrm{keV}$ passes all of the cuts. . . . . . . . . . . . . . . . . . . . . 108 
7.2 Exclusion limit from this analysis. The thick solid line corresponds to the lower bound of points excluded at $90 \%$ confidence. The yellow band corresponds to the region which contains the sensitvity for $50 \%$ of an ensemble of simulated experiments with our expected backgrounds listed in Table 6.1. . . . . . . . . . . . . . . . . . . . . . . . 109

7.3 Plot of $\sigma_{S I}$ per nucleon versus WIMP mass. Exclusion curves for EDELWEISS (black dashed) [40], CDMS I - SUF (blue dashed) [41, 43], CDMS II - SUF (blue solid) [42, 37], ZEPLIN (magenta solid), CRESST (magenta dashed), and CDMS II - Soudan (black solid). The red region is the DAMA detection claim [39]. Limitplot courtesy of [44].110

7.4 Limit plot showing allowed regions corresponding to allowed models from Fig. 1.8[32] (cyan), recent mSUGRA framework calculations using Markov chain monte carlos (dark blue) [67], and split supersymmetry (yellow dots). The black curve is the current Soudan Run 118 limit. The dashed curve is the expected final CDMS II Soudan sensitivity. The red region is the DAMA detection claim [39]. Limitplot courtesy of $[44] \ldots \ldots \ldots \ldots \ldots \ldots \ldots \ldots$ 


\section{Chapter 1}

\section{Introduction}

\subsection{Hints of Physics Beyond the Standard Model}

The Standard Model of particle physics is one of the triumphs of 20th century physics. It is a gauge theory based on the $S U(3)_{C} \times S U(2)_{L} \times U(1)_{Y}$ gauge group where interactions between three generations of quarks and leptons are mediated by gauge bosons. Particles come to have mass via the Higgs mechanism since explicit mass terms in the Lagrangian would violate gauge invariance. The Higgs mechanism introduces a single scalar $S U(2)$ doublet which sits in a "Mexican hat" potential

$$
V(\phi)=-\mu^{2} \phi^{2}+\lambda \phi^{4}
$$

and has Yukawa couplings to fermions. Eqn. (1.1) gives the Higgs field a non-zero vacuum expectation which "breaks" gauge invariance. It is the non-zero vacuum expectation of the Higgs field that then gives mass to all other particles.

There are many suggestions that the Standard Model is incomplete. In this section, I will present two problems that point towards physics beyond the Standard Model and discuss an attractive extension that addresses both of these issues.

\subsubsection{The Gauge Hierarchy Problem}

The first problem is the Gauge Hierarchy problem and is theoretical in nature. 
Though the Higgs mechanism is appealing from the standpoint of preserving the gauge symmetry, it has a few problems ${ }^{1}$. One of the problems is the Gauge Hierarchy problem. If we consider first order quantum corrections to the Higgs self energy, we find that the fermion Yukawa couplings lead to diagrams like those shown in Fig. 1.1. The contribution of such loops to the Higgs mass diverges quadratically

$$
\Delta \mu^{2} \sim \lambda_{f}^{2} \Lambda^{2}
$$

where $\lambda_{f}$ is the Yukawa coupling of the fermion $f$ and $\Lambda$ corresponds to the cutoff from which new physics must enter. In the Standard Model, the radiative corrections are dominated by loops with the top quark, the $W$ bosons, and the Higgs itself.

Natural values for $\Lambda$ would be $M_{G U T}$ or $M_{\text {Planck }}$. The problem with the quadratic divergence is that Electro-Weak symmetry breaking gives

$$
\langle\phi\rangle=\sqrt{\frac{\mu^{2}}{\lambda}} \sim 200 \mathrm{GeV}
$$

which is only possible if extreme fine tuning cancels the corrections of order $M_{\text {Planck }}^{2}$ from Eqn. 1.8. One might think that this problem would extend to the gauge bosons as well since they also receive corrections from diagrams similar to Fig. 1.1. However, in the case of the gauge bosons, they are protected by gauge invariance and diagrams cancel leaving only a logarithmic divergence. The removal of quadratic divergences for the gauge bosons by a symmetry presents an idea as to how the Gauge Hierarchy problem may be resolved.

\subsubsection{The Problem of Dark Matter}

The second indicator of physics beyond the Standard Model is the mounting evidence for non-baryonic dark matter for which the Standard Model has no explanation. For reviews of this evidence, the reader is referred to $[1,2,3]$. In this section, I will briefly summarize two sets of observations that are especially relevant to CDMS II.

\footnotetext{
${ }^{1}$ One problem, that we will not discuss, is that neither the Higgs nor any other fundamental scalar has been observed.
} 


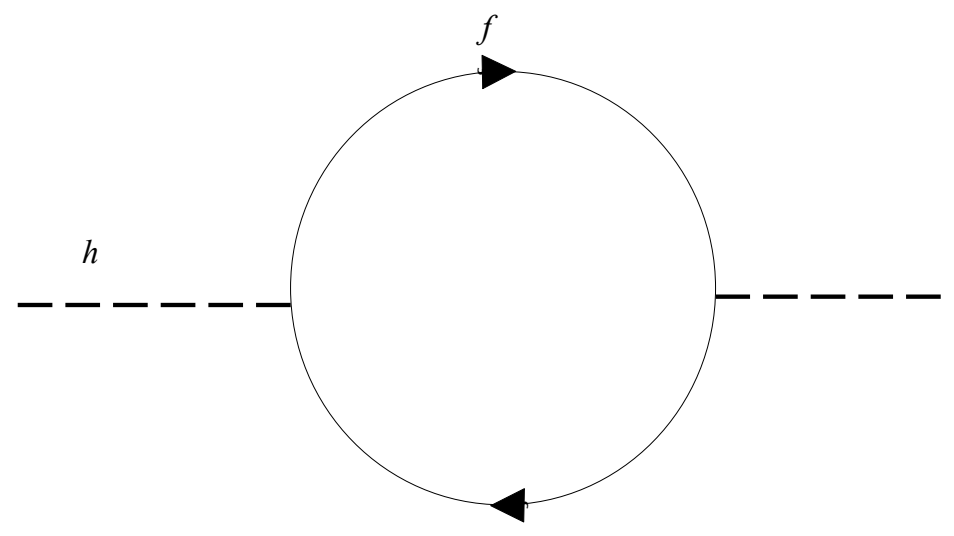

Figure 1.1: Fermion loop correction to the Higgs self energy. There are also corrections from loops with the gauge bosons and the Higgs itself. In the Standard Model, the $t, W$, and $h$ loops give the largest contributions.

\section{Galactic Dark Matter}

The first set of observations deal with dark matter in spiral galaxies. It is possible to estimate the spatial mass distribution in a spiral galaxy by measuring the rotational velocity for objects that would be subject to the galaxy's gravity. Specifically, we can determine the rotational veolocity of clouds of neutral hydrogen by measuring the Doppler shift of the fine structure line.

If the majority of the mass of a galaxy is in the form of luminous matter, Keplerian mechanics predicts that the rotational velocity for objects far from the luminous part of a spiral galaxy should decrease as $1 / \sqrt{r}$. However, the measured rotation curves plateau instead of falling off indicating that there is substantial mass where there is no significant luminous matter. Fig. 1.2 shows the rotation curve of a typical spiral galaxy and Fig. 1.3 shows that this profile is characteristic of many spiral galaxies. The rotation curves suggest that the majority of the mass in a spiral galaxy is actually 


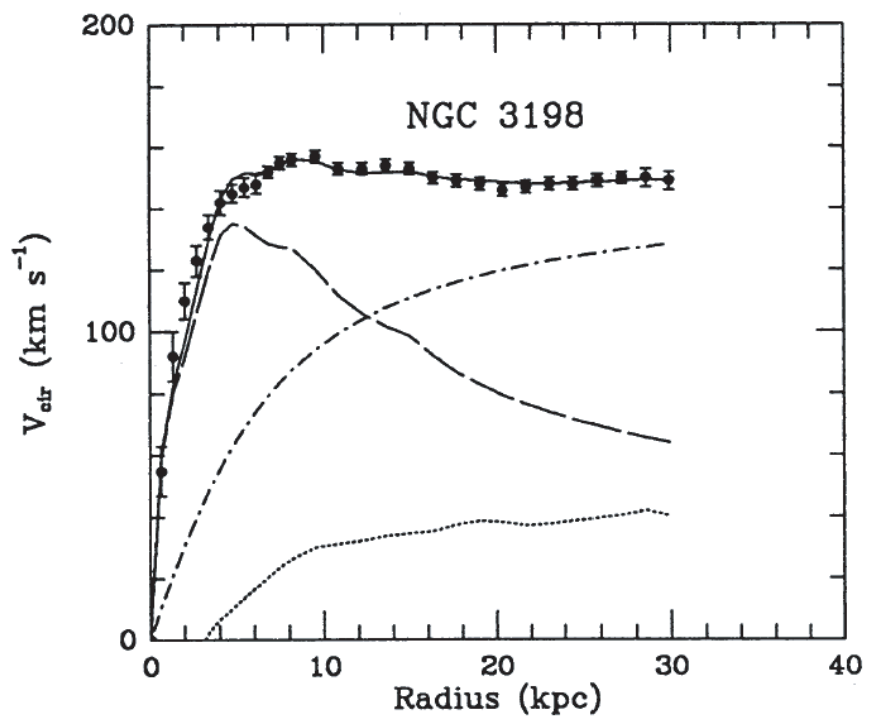

Figure 1.2: Galactic rotation curve for galaxy NGC3198 [4]. The non-Keplerian behavior of the flat rotation curve (instead of $1 / \sqrt{r}$ ) indicates a large fraction of the galactic mass is non-luminous. The dashed line corresponds to the mass distribution of visible matter. The dotted line corresponds to the distribution of gas. The dotdashed line corresponds to the mass distribution of the non-luminous halo.

in the form of a non-luminous halo.

The entire dark matter halo may be comprised of normal baryonic matter that is in a form that we cannot see. The current favored dark baryon objects are Massive Compact Halo Objects (MACHOs). Two experiments $[6,7]$ have undertaken searches for MACHOs which can be observed through gravitational microlensing of distant stars. Both experiments observe a number of events that indicate the presence of MACHOs, however, neither experiment finds a sufficient number of events to support the claim that the majority of the dark matter halo is comprised of MACHOs. Fig. 1.4 shows exclusion plots from a combined analysis of the data from both experiments [8] which indicates that less than $25 \%$ of the galactic halo is in the form of MACHOs with masses $10^{-7} \mathrm{M}_{\odot} \lesssim m \lesssim 10^{-3} \mathrm{M}_{\odot}$. These measurements suggest that most of the dark matter halo must be in the form of an exotic particle that is not currently part of the Standard Model. 

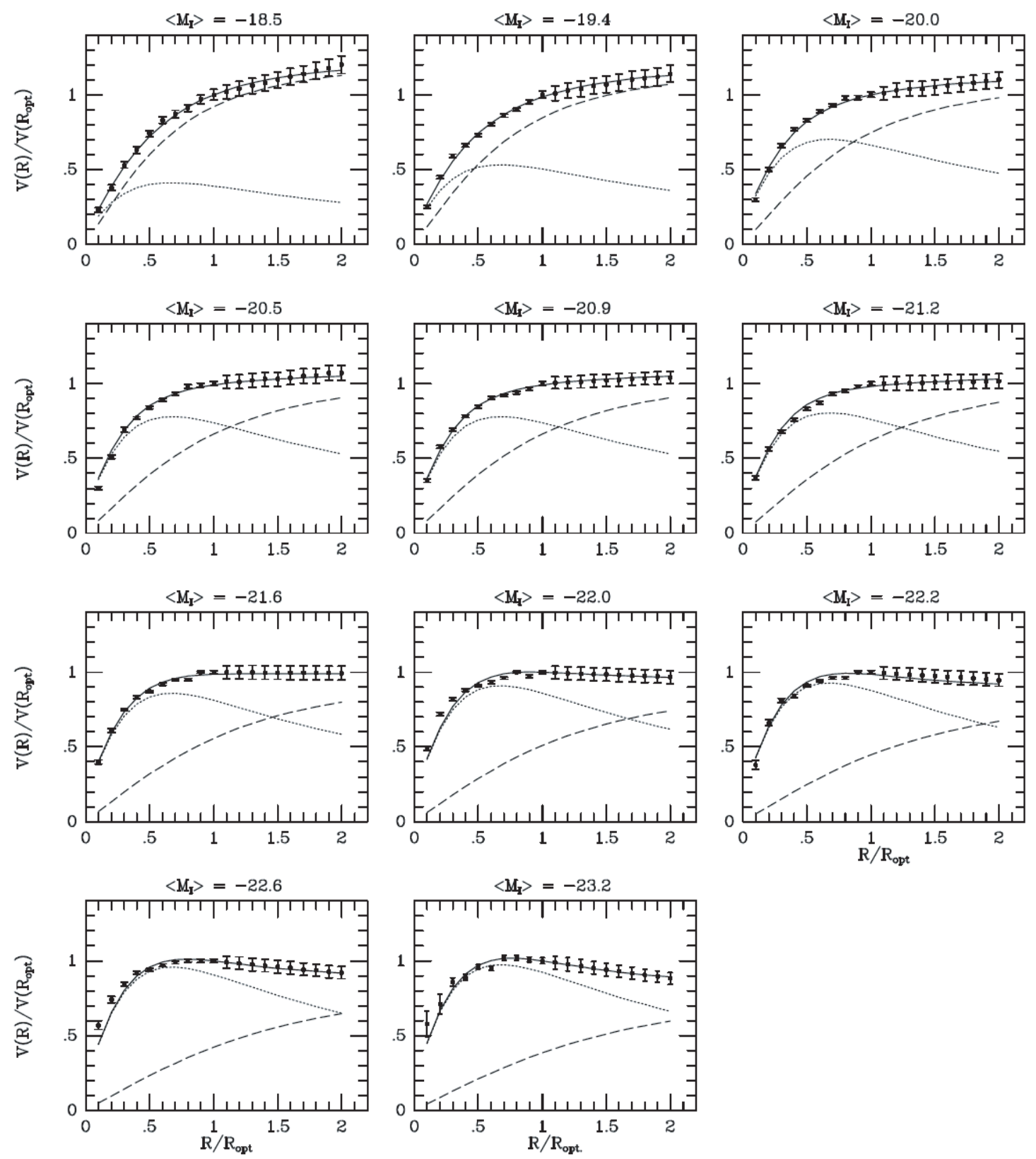

Figure 1.3: Average rotation curves for 1000 spiral galaxies. Each plot shows the average rotation curve (solid line) for galaxies with the indicated luminosities (in absolute magnitude). The dotted lines corresponds to the contribution from the visible disc and the dashed lines correspond to the contribution from a dark halo. Figure from [5]. 


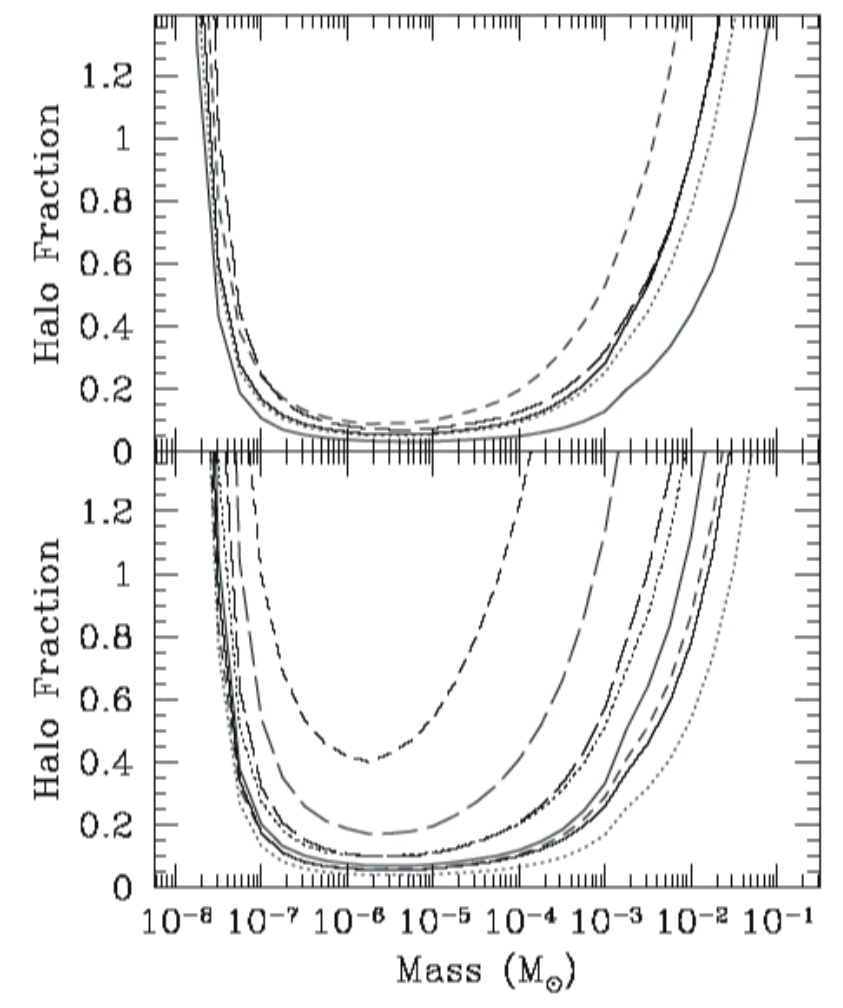

Figure 1.4: Exclusion limits for combined data from EROS and MACHO [8]. The top figure shows exclusion curves for five models considered by EROS [7] and the bottom figure shows exclusion curves for the eight models considered by MACHO [6]. 


\section{CMB and LSS Power Spectra}

The second set of observations indicating the existence of non-baryonic dark matter comes from observations of the power spectra for the Cosmic Microwave Background [9] and large scale structure [10]. Tegmark et al. [11] have performed a combined analysis of these power spectra. The CMB and LSS power spectra are extremely sensitive to many cosmological parameters. Precise measurements of both power spectra point to a universe that is flat with $\Omega_{m}=0.3$, where $\Omega_{m}$ is the matter density divided by the critical density. ${ }^{2}$ The measurements also measure the baryon density $\Omega_{b} h^{2}=0.023$ where the Hubble constant $H_{0}=100 \cdot h$. Comparing this measurement to the total mass density $\Omega_{m} h^{2} \sim 0.12$ reveals that more than $80 \%$ of the matter density must be non-baryonic.

Additionally, the power spectra indicate that most of this dark matter needs to be non-relativistic or "cold" at the time when matter comes to dominate the energy density of the universe, making heavy particles attractive as dark matter candidates. $N$-body simulations $[12,13]$ of hierarchical structure formation with cold dark matter $[14,15]$ give reasonable agreement with the observed LSS power spectrum [16]. In hierarchical structure formation, larger structures emerge from the combination of smaller structures. "Hot dark matter," such as neutrino dark matter, is currently disfavored since it would wash out small scale density perturbations [17] requiring that structure form through "top-down" processes where larger structures fragment into smaller ones [18].

\section{Relic Abundances}

The precise measurements from experiments such as WMAP and SDSS make it possible to make guesses as to the nature of the dark matter. Of particular relevance is the measurement of the dark matter relic abundance density, $\omega_{d}=\Omega_{d} h^{2} \sim 0.1$. Lee and Weinberg [19] initially used upper bounds on $\omega_{d}$ to put constraints on the mass of heavy neutrino dark matter. I will give a quick summary of their argument and

\footnotetext{
${ }^{2}$ The measurements also measure the existience of dark energy which has a density $\Omega_{\Lambda}=0.7$. The dark energy problem is a substantial mystery for which I have a solution, but there isn't room to discuss that here.
} 


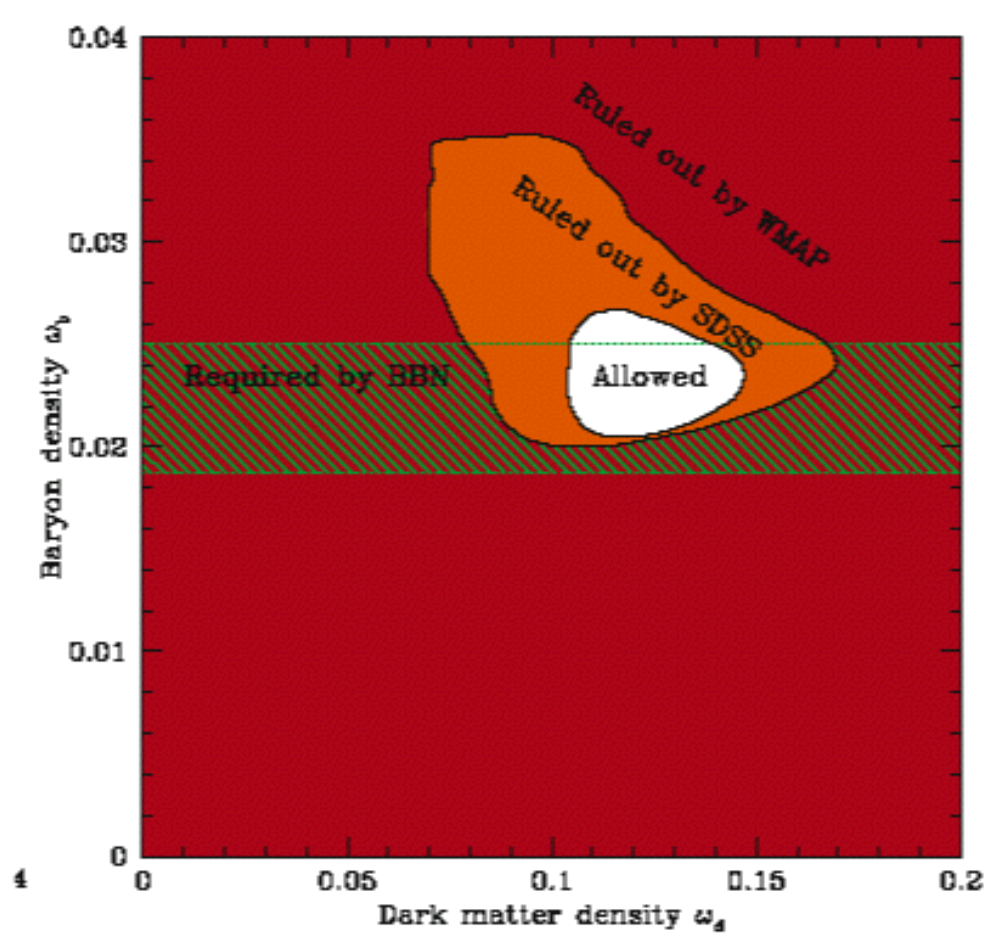

Figure 1.5: Excluded regions in the $\left(\omega_{b}, \omega_{d}\right)$ plane for combined SDSS and WMAP analysis. $\omega_{b}=\Omega_{b} h^{2}$ is the baryonic density and $\omega_{d}=\Omega_{d} h^{2}$ is the nonbaryonic matter density [11].

discuss its implications for our current understanding of particle dark matter.

The evolution of the number density $n_{\chi}$ for a particle $\chi$ that was produced in thermal equilibrium in the early universe is given by the Boltzman equation

$$
\frac{d n_{\chi}}{d t}+3 H n_{\chi}=-\left\langle\sigma_{A} v\right\rangle\left[\left(n_{\chi}\right)^{2}-\left(n_{\chi}^{e q}\right)^{2}\right]
$$

where $\langle\cdots\rangle$ denotes thermal averaging, $H$ is the scale factor, $\sigma_{A}$ is the annihilation cross section, $v$ is the velocity, and $n_{\chi}^{e q}$ is the density at thermal equilibrium.

$$
n_{\chi}^{e q} \propto\left\{\begin{array}{cl}
T^{3} & T \gg m_{\chi} \\
T^{3 / 2} \exp \left(\frac{-m_{\chi}}{T}\right) & T \ll m_{\chi}
\end{array}\right.
$$

The first term on the right hand side of Eqn. 1.3 corresponds to depletion from annihilation. The second term on the right hand side corresponds to the creation of 
$\chi_{\mathrm{s}}$ from particles in the thermal background. The second term on the left hand side accounts for dilution due to the expansion of the universe. When the annihilation rate, $\left\langle\sigma_{A} v\right\rangle n_{\chi}$, drops below the Hubble expansion rate, $H$, the $\chi$ particles become too dilute to significantly annihilate. The result is that $n_{\chi}>n_{\chi}^{e q}$ making it possible to have a significant relic abundance of a massive particle. This phenomenon is known as "freeze out."

Lee and Weinberg showed that if the mass of a heavy neutrino is too low, the relic abundance remaining after freezing out would be too large. We can use a variation of the argument. Given that there is a significant well measured relic abundance and assuming that the dark matter is massive and therefore cold, then the scale of the annihilation rate must be comparable to that of Weak physics.

We can work out a crude estimate. Barring any exotic entropy producing physics, the relic abundance today is

$$
\Omega_{\chi} h^{2}=\frac{m_{\chi} n_{\chi}}{\rho_{c}}=\frac{s_{0}}{\rho_{c}} m_{\chi}\left(\frac{n_{\chi}}{s}\right)_{f}
$$

where $\rho_{c}$ is the critical density, $s_{0}$ is the entropy density today, and the $f$ subscript denotes values at freeze out. At the time of freeze out, the universe was radiation dominated giving $H^{2} \propto g T^{4}$ and $s_{f} \propto g T^{3}$ where $g$ is the number of relativistic degrees of freedom. The freeze out criteria is $H=\left\langle\sigma_{A} v\right\rangle n_{\chi}$, so we have

$$
\Omega_{\chi} h^{2} \propto \frac{1}{\sqrt{g}\left\langle\sigma_{A} v\right\rangle n_{\chi}}\left(\frac{m_{\chi}}{T_{f}}\right)
$$

For numbers typical of weak physics, numerical solutions for the freeze out criteria give $m_{\chi} / T_{f} \sim 20$ up to logarithimic corrections. Thus, plugging in all numbers, we get

$$
0.1 \simeq \Omega_{\chi} h^{2} \simeq \frac{3 \times 10^{-27} \mathrm{~cm}^{3} \mathrm{~s}^{-1}}{\left\langle\sigma_{A} v\right\rangle n_{\chi}}
$$

indicating that $\left\langle\sigma_{A} v\right\rangle$ is of the order of Weak annihilation rates.

In summary, the measurements of the relic abundance of non-baryonic dark matter are consistent with the existence of particles that are massive and posses interactions on the same scale as Weak physics. Such particles are usually called WIMPs (Weakly 


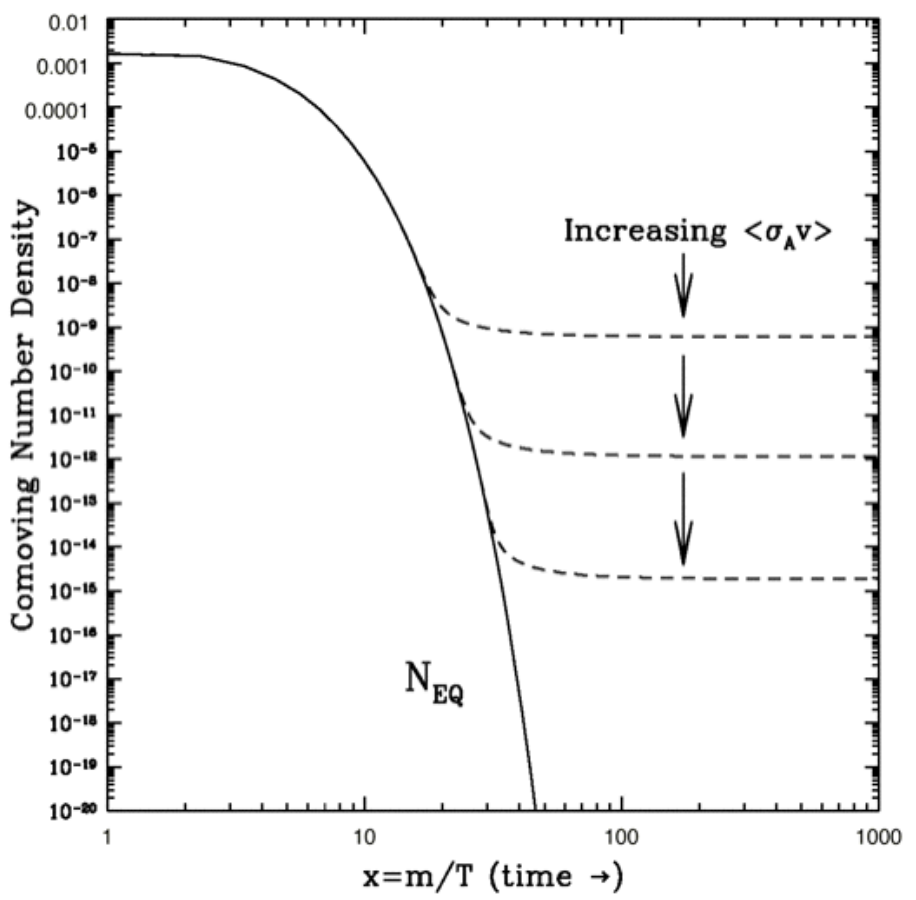

Figure 1.6: The number density of a stable particle versus time. When $\left\langle\sigma_{A} v\right\rangle=H$, the particle freezes out and the number density remains constant. The solid line corresponds to the equilibrium density. The dashed lines indicate the relic abundance for particles with different annihilation rates. Particles with larger annihilation rates freeze out later leaving a smaller relic abundance. Figure from [20].

Interacting Massive Particles).

\subsection{Supersymmetry}

\subsubsection{Preliminaries}

Supersymmetry refers to a continuous symmetry that transforms fermions into bosons and vice versa. An infinitesimal supersymmetric transformation between a scalar field $\phi$ and a fermion field $\psi$ has the form 


$$
\begin{aligned}
& \delta \phi=-\imath \epsilon^{T} \sigma^{2} \psi, \\
& \delta \psi=\epsilon F+\sigma \cdot \partial \phi \sigma^{2} \epsilon^{*}, \\
& \delta F=-\imath \epsilon^{\dagger} \bar{\sigma} \cdot \partial \psi
\end{aligned}
$$

where $\epsilon$ is an infinitesimal spinor object of Grassmann numbers which parameterizes the transformation and $F$ is an auxilary field with no degrees of freedom. $F$ serves only as a mathematical book keeping tool. The particles $\phi$ and $\psi$ are considered superpartners of each other since they are related by a supersymmetric transformation. It can be shown that as long as supersymmetry is maintained, $\phi$ and $\psi$ must have the same mass. For a more detailed introduction to supersymmetry, the reader is referred to [21].

\subsubsection{The MSSM Framework}

Since supersymmetry requires a large spectrum of additional interactions and particles, there is a lot of freedom in how one might extend the Standard Model to make it supersymmetric. We shall restrict ourselves to a class of supersymmetric models that "just barely" extend the Standard Model. Generally referred to as the Minimal Supersymmetric Standard Model framework, these models only introduce enough particles to make the Standard Model supersymmetric. The not so obvious part of a minimal supersymmetric extension to the Standard Model is that 2 Higgs fields (together with their superpartners) are required [21].

An additional constraint for the MSSM is the conservation of R-parity. R-parity conservation forbids interactions that would lead to regular particles decaying into sparticles and vice versa. Such interactions lead to baryon and lepton number violation. One especially relevant consequence of R-parity conservation is that the Lightest Supersymmetric Partner (LSP) is forbidden from decaying.

A final constraint on the MSSM framework is that supersymmetry must be broken. Unbroken supersymmetry has the property that particles and their superpartners 


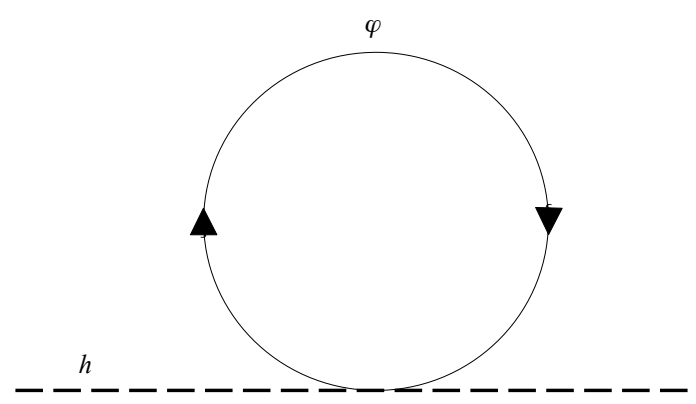

Figure 1.7: A boson loop contribution to the Higgs self energy that would exactly cancel the contribution from its fermion superpartner shown in Fig. 1.1.

must have the same mass. Since we clearly do not observe this phenomenon, our current state must be one of broken supersymmetry. This last requirement on the MSSM is not extremely detailed in that it doesn't really care how supersymmetry is broken. This flexibility introduces quite a bit of freedom. When all constraints for the MSSM are considered, there are 108 parameters in addition to those of the Standard Model.

\subsubsection{SUSY and the Gauge Hierarchy Problem}

Supersymmetry provides a natural resolution of the Gauge Hierarchy problem described in Sec. 1.1.1. In a supersymmetric theory, every fermion loop contribution to the Higgs self energy would have a corresponding boson loop (shown in Fig. 1.7) from the fermion's superpartner. In unbroken supersymmetry, the superpartners have the same mass and would exactly cancel the contributions from the fermion loops. These cancellations would occur at all orders in perturbation theory since they will be a consequence of a symmetry much like the case with the gauge bosons of the Standard Model.

In the MSSM framework, supersymmetry is broken allowing superpartners to 
have different masses which makes the cancellation imperfect. However, the radiative corrections to the Higgs mass do not have a quadratic dependence on the cutoff. They are of the form

$$
\Delta \mu^{2} \sim \Delta m^{2} \lambda \ln \Lambda
$$

where $\lambda$ is the Yukawa coupling, $\Lambda$ is the cutoff, and $\Delta m$ corresponds to the mass difference between the superpartners. Fine tuning of the Higgs mass can still be avoided as long as $\Delta m$ is not extremely large. $(<1 \mathrm{TeV})$.

\subsubsection{SUSY and Dark Matter}

Supersymmetry also provides an attractive solution to the dark matter problem. In the case where R-parity is conserved, the Lightest Supersymmetric Partner (LSP) must be stable. Thus, superparticles produced during the big bang would decay to the LSP which would remain until today. It turns out that in many supersymmetric models, the LSP has properties that are consistent with our understanding of dark matter. For a more detailed discussion of supersymmetric dark matter, the reader is referred to [22].

\section{The Neutralino}

The currently favored supersymmetric dark matter candidate is the neutralino. The neutralino is a combination of the fermion partners of the photon, $Z^{0}$, and the two neutral Higgs bosons. Since the neutralino is massive and does not couple directly to the photon, it has precisely those qualities which are required for a cold dark matter candidate. Additionally, the tree level Feynman diagrams for neutralino annihilation all involve the exchange of particles with masses of $O(100 \mathrm{GeV})$ or higher, which sets the scale of the annihilation cross section to be comparable to that of Weak physics. Weak scale annihilation rates imply that neutralinos would have a relic abundance that is close to what is measured by the CMB and LSS power spectra. 


\section{Other Dark Matter Candidates}

There are a number of other well motivated dark matter candidates in addition to the neutralino, not all of which require supersymmetry. It is worthwhile to briefly mention a few of these other candidates together with their motivations.

- neutrinos: Recent measurements of the oscillations of the three neutrinos of the Standard Model indicate that they are not massless [23, 24, 25]. However, the neutrinos would not be massive enough to form dark matter that is cold. One possible dark matter candidate would be an extremely heavy neutrino that is sterile and only couples to other particles via neutrino mixing. Such a particle is very well motivated if one desires to explain the measurements of LSND [26] which measure a third mass splitting for neutrino oscillations.

- axions: Axions are particles motivated by the need to solve the strong-CP problem. Axions couple extremely weakly to ordinary matter, and thus rapidly fall out of thermal equilibrium. Though constrained to be light by accelerators and astrophysical arguments, the axions are still attractive as cold dark matter because their mass is intimately tied to the QCD phase transition and the mechanism through which the axion addresses the strong-CP problem. In short, when the universe cools below the QCD phase transition, the axions produced are cold.

- Other SUSY candidates: Supersymmetric extensions of the Standard Model have other dark matter candidates. The superpartner of the neutrino, the sneutrino, was favored for some time. However, the coupling of the sneutrino to nuclei is well constrained. The lack of detection of the sneutrino by elastic scattering off of nuclei currently disfavors the sneutrino as dark matter [27]. Other supersymmetric dark matter candidates are the axino [28] and gravitino both of which are difficult to detect due to extremely weak coupling to ordinary matter. 


\subsubsection{Accelerator and Cosomological Constraints on MSSMs}

There are a number of experimental constraints on the MSSM that come from measurements at accelerators.

- Direct sparticle searches: Accelerators can search for supersymmetric particles directly. No evidence for sparticles has been found setting severe lower bounds on the mass of the LSP [1].

- Flavor changing neutral currents: Particles with large masses can have significant impact on other low energy measurements through radiative corrections. The effects of these quantum corrections can be most effectively detected through precise measurements of rare decay processes such as flavor changing neutral currents. The most severe constraint comes from measurements of $b \rightarrow s \gamma[29,30]$.

- Anomalous Muon Magnetic Moment: The recent precision measurement of ( $g-$ $2)_{\mu}$ gives a result that differs from the Standard Model prediction by either $1.6 \sigma$ or $3 \sigma$ [31]. This measurement can be used to further constrain MSSMs. In particular, it constrains the Higgsino-mass parameter, $\mu$, to be positive.

Further constraints arise if the neutralino is to comprise the majority of the dark matter in the universe. If this is the case, then the neutralino must be the LSP and the model must yield a neutralino relic abundance that is consistent with the measured relic abundance of cold dark matter. Fig. 1.8 shows the reduction in the MSSM parameter space when all of the above constraints are considered.

\subsection{Direct Detection of Dark Matter}

Goodman and Witten realized that it may be possible to detect the galactic dark matter through elastic scattering of the dark matter off of nuclei [33]. In this section, I will briefly discuss the expected signal for elastic scattering of neutralinos and describe some of the current direct detection experimental efforts. For more information, the reader can consult $[34,35]$. 

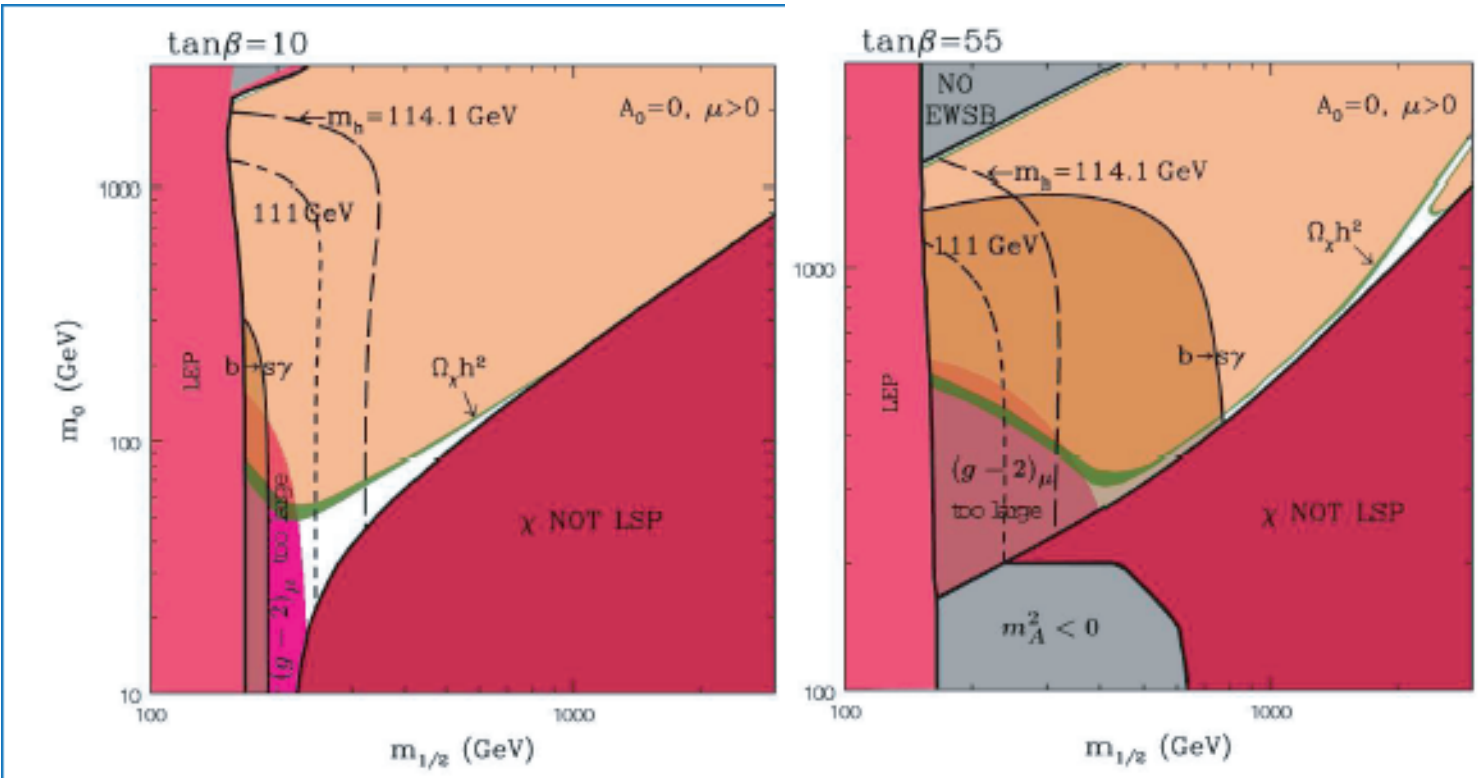

Figure 1.8: Exclusion plot of various MSSM theories for parameters in the $\left(m_{0}, m_{1 / 2}\right)$ plane for $\tan \beta=10$ (left) and $\tan \beta=55$ (right). $m_{1,2}$ is the GUT gaugino unification mass, $m_{0}$ is the mass of the pseudoscalar Higgs. For both plots $\mu>0$. Note the light green region indicating the relic abundance constraints [32].

\subsubsection{Expected Signal}

The expected rate of scattering per unit detector mass is given by

$$
d R=\frac{1}{m_{N}}\left(\frac{d \sigma}{d q^{2}}\right)\left(\frac{\rho}{m_{\chi}} v f(v)\right) d q^{2} d^{3} v
$$

where $m_{N}$ is the mass of the target nucleus, $\rho$ is the local density of the neutralino, $m_{\chi}$ is the neutralino mass, $d \sigma / d q^{2}$ is the differential cross section for scattering of the neutralino off of the nucleus, and $f(v)$ is the velocity distribution of the neutralinos. $q^{2}$ is the momentum transferred in the scattering with $q^{2}=2 m_{r}^{2} v^{2}(1-\cos \theta)$ where $\theta$ is the scattering angle in the center of mass frame. The reduced mass is $m_{r}=$ $m_{N} m_{\chi} /\left(m_{N}+m_{\chi}\right)$ and the total recoil energy is just $Q=q^{2} / 2 m_{r}$. Eqn. (1.9) shows that there are two contributions to the expected rate. The first contribution, $d \sigma / d q^{2}$, contains all of the physics associated with the interaction of the neutralino with 
matter. The second contribution, $\frac{\rho}{m_{\chi}} v f(v)$ deals with the astrophysical distribution of the neutralinos in our galaxy.

\section{Interaction of Neutralinos with Matter}

Determining the differential neutralino-nucleus cross section involves calculating the neutralino-nucleon cross section and then summing this cross section over the structure of the nucleus. The neutralino-nucleon cross section contains all of the dependence on the particle physics model. Since the scattering is non-relativistic, the neutralino coupling to matter breaks down into two components. The first component is an effective scalar interaction which couples to nucleon number and the second component couples to the nucleon spin. This decomposition is a general one, appropriate for any particle in the non-relativistic limit [36].

Since, the majority of the nuclei in the CDMS detectors have no net nuclear spin, the CDMS experiment is less sensitive to the spin dependent component of the interaction. For this reason, I will only discuss the consequences of the scalar or spin-independent interaction.

The scalar neutralino-nucleon cross section is dominated by two processes: interactions with quarks in the nucleon through Higgs and squark exchange, and interactions with gluons in the nucleon through quark and squark loops. Fig. 1.9 shows some of the Feynman diagrams contributing to the scalar cross section. Direct evaluation of the Feynman diagrams and nucleon matrix elements give the matrix elements for the scattering of the neutralino off of protons, $f_{p}$, and neutrons, $f_{n}$. If we assume that all the nucleons in the nucleus add coherently, the differential neutralino-nucleus cross section is given by Fermi's Golden rule

$$
\frac{d \sigma}{d q^{2}}=\frac{1}{\pi v^{2}}\left(Z f_{p}+(A-Z) f_{n}\right)^{2}
$$

where $A$ is the total number of nucleons and $Z$ is the number of protons. In most models, $f=f_{p} \simeq f_{n}$ which gives

$$
\frac{d \sigma}{d q^{2}}=\frac{1}{\pi v^{2}} A^{2} f^{2}
$$



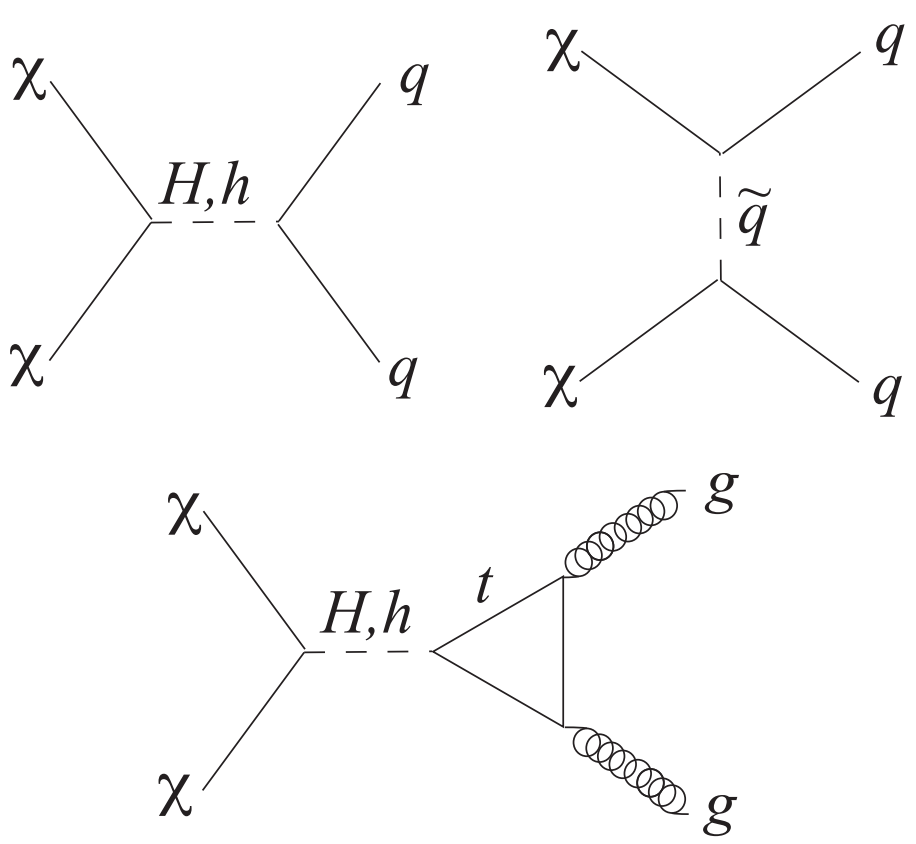

Figure 1.9: Some of the Feynman diagrams contributing to the spin independent neutralino nucleon cross section. For some models, couplings of the neutralino to gluons through quark and squark loops becomes important.

The assumption that all the nucleons add coherently is valid only in the limit of zero momentum transfer. For non-zero momentum transfer, the loss of coherence is usually accounted for through a form factor $F(Q)$. Typically, one uses the WoodsSaxon form factor for $F(Q)$

$$
F(Q)=\left[\frac{3 j_{1}\left(q R_{1}\right)}{q R_{1}}\right] \exp \left(-q s^{2} / 2\right)
$$

with $R_{1}=\sqrt{R^{2}+5 s^{2}}, R \sim 1.2 A^{1 / 3} \mathrm{fm}$, and $s \sim 1 \mathrm{fm}$, and a spherical Bessel function

$$
j_{1}(x)=\frac{\sin (x)-x \cos (x)}{x^{3}}
$$


Fig. 1.10a shows the Woods-Saxon form factor for a few target nuclei.

Putting it all together gives

$$
\frac{d \sigma}{d q^{2}}=\frac{1}{\pi v^{2}} A^{2} f^{2} F^{2}(Q)
$$

There are two points that are worth noting. The first is that since the scalar cross section couples to nucleon number, there is a quadratic gain in scattering off of larger nuclei. This gain however, is partially negated by the rapid loss of coherence for a larger nucleus. Thus, in order to maximize the gain from using large nuclei, experiments will need to operate at extremely low recoil thresholds (see Fig. 1.10).

\section{Astrophysical Distribution of WIMPs}

The expected recoil spectrum depends on astrophysics through

$$
\frac{\rho}{m_{\chi}} v f(v)
$$

Recent detailed simulations of galaxy formation [38] indicate that it is reasonable to assume that the velocity distribution, $f(v)$, is Maxwellian with a non-zero central value and that the density of dark matter, $\rho$, is uniform. Typical values are $\rho=0.3$ $\mathrm{GeV} \mathrm{cm}{ }^{-3}$ and a velocity dispersion $v_{0}=220 \mathrm{~km} \mathrm{~s}^{-1}$. These values for the density and the velocity distribution are extracted from rotation curve measurements described in Sec. 1.1.2 and are subject to a significant amount of uncertainty.

We can obtain a qualitative feel for the shape of the recoil spectrum by ignoring the velocity of the earth and the escape velocity of the neutralinos. The recoil spectrum is

$$
\frac{d R}{d q^{2}}=\frac{1}{\pi} A^{2} f^{2} F^{2}(Q) \frac{f(v)}{v} d^{3} v
$$

Taking $f(v)$ to be Maxwellian and integrating over the velocity distribution gives

$$
\frac{d R}{d q^{2}} \propto A^{2} F^{2}(Q) \exp \left(-\frac{Q m_{N}}{2 m_{r}^{2} v_{0}^{2}}\right)
$$

Thus, the recoil spectrum from elastic scattering of neutralinos off of nuclei is expected 


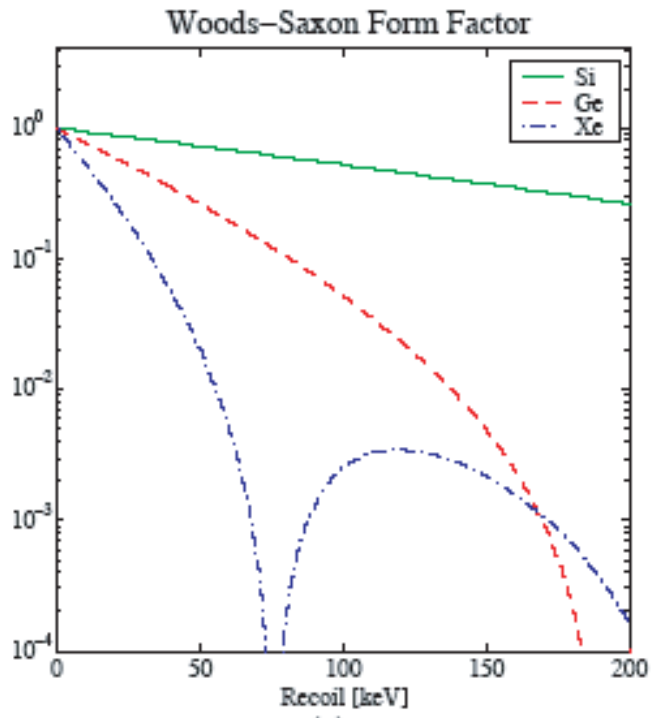

(a)

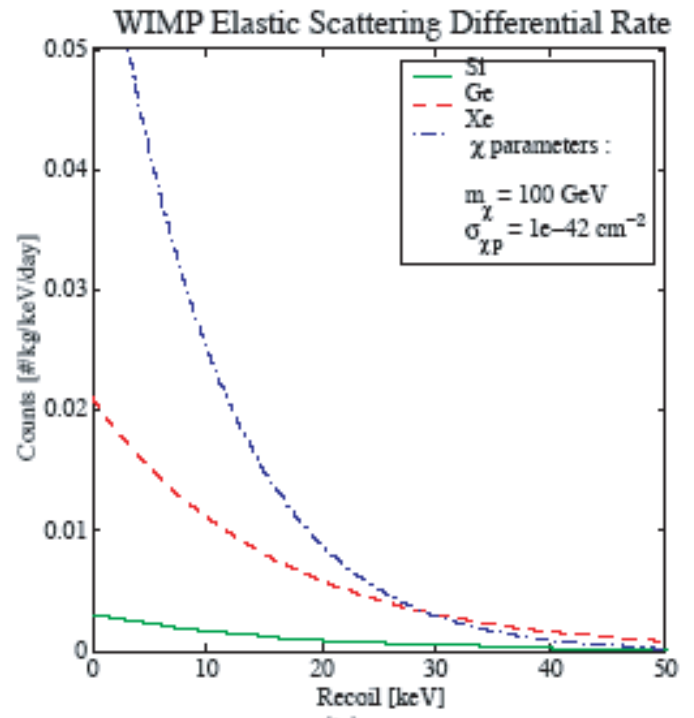

(b)

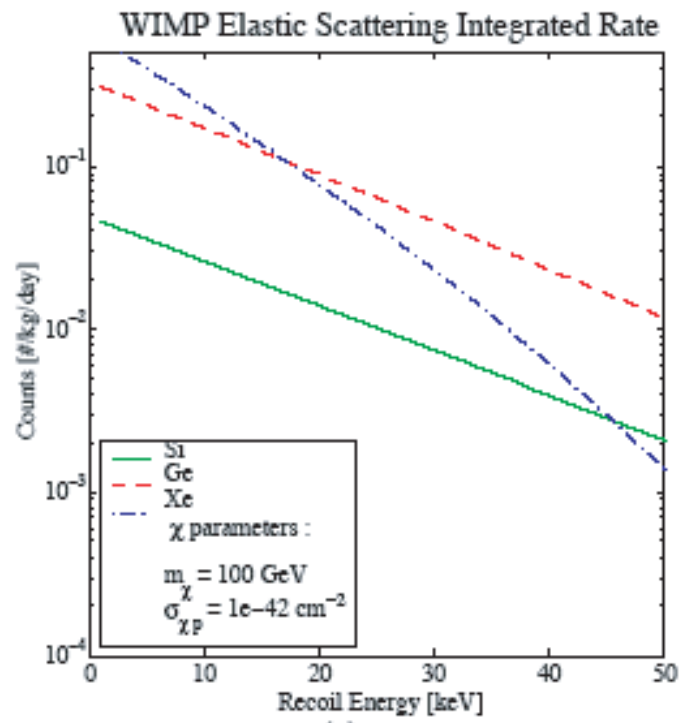

(c)

Figure 1.10: (a) The Woods-Saxon form factor for Ge, Si, and Xe. (b) The differential WIMP elastic scattering rate for Ge, Si, and Xe. (c) The integrated WIMP elastic scattering rate for Ge, Si, and Xe. Figure from [37]. 
to be a featureless spectrum that falls off very rapidly. Putting in numbers gives event rates of $<1$ event $\mathrm{kg}^{-1}$ day $^{-1}$ with typical recoil energy of a few to tens of $\mathrm{keV}$.

\subsubsection{Some Current Direct Detection Experiments}

The extremely low rates and recoil energies place severe constraints on experiments searching for neutralino dark matter through elastic scattering off of nuclei. The experiments must have extremely low backgrounds and low thresholds. The primary backgrounds for these experiments come from radioactive contaminants and cosmic rays. In order to reduce these backgrounds, most experiments are operated in low radioactivity environments deep underground. Additionally, the lack of any spectral features makes it worthwhile for experiments to have an additional way of demonstrating that a signal is due to interaction with galactic dark matter.

\section{DAMA}

The DAMA collaboration uses NaI as their target mass and measures the recoil energy by measuring scintillation in the crystal. For this dark matter search, the signature of interaction with dark matter is the annual modulation of the recoil spectrum. When we include the motion of the earth through the galaxy, we find that the expected recoil spectrum should be harder when the earth is moving with the solar system through the galaxy and softer when the earth is moving against the solar system. The DAMA collaboration has taken seven years of data for a total exposure of 58,000 $\mathrm{kg}$ days. There is clear evidence for an annual modulation of their signal which the DAMA collaboration claims to be due entirely to dark matter. This experiment is the only experiment to claim a dark matter detection [39]. Fig. 1.11 shows the allowed region of neutralino mass and scalar nucleon cross section for their detection.

\section{ZEPLIN}

ZEPLIN uses liquid Xe as its target mass and discriminates between nuclear recoils and electron recoils. Dark matter interactions will only scatter off of target nuclei while the majority of known backgrounds will scatter off of electrons in the target 
mass. ZEPLIN only measures the scintillaton produced in the Xe. An electron recoil produces more ionization which releases light when the free electron recombines with the Xe. The slight delay due to recombination makes it possible for a signal due to nuclear recoils to be distinguished from a signal due to electron recoils through statistical pulse shape analysis. The ZEPLIN experiment does not see any nuclear recoil signal. However, the robustness of this result is sometimes questioned due to the lack of an in-situ neutron calibration.

\section{EDELWEISS and CDMS I}

The detector technology for EDELWEISS and CDMS I is virtually identical. The target mass is Ge. Electrodes on the detector surface measure the ionization produced by a recoil while thermistors simultaneously measure the heat that is produced. These detectors can discriminate between nuclear recoils and electron recoils on an event by event basis since the heat measurement is an absolute measurement of the recoil energy while the ionization is reduced or quenched for nuclear recoils. The detectors have one electromagnetic background. Electron recoils near the electrodes can have deficient charge collection resulting in reduced ionization measurements. Neither experiment observes a signal from dark matter [40, 41]. The sensitivity of CDMS I was limited by an irreducible cosmogenic neutron background at the Stanford Underground Facility.

\section{CRESST}

The target mass of CRESST is a crystal of $\mathrm{CaWO}_{4}$. CRESST simultaneously measures both the scintillation and the athermal phonons produced by a recoil. Discrimination between nuclear and electron recoils is possible since the scintillation signal is reduced for nuclear recoils. Unlike the detectors for CDMS and EDELWEISS, the CRESST detector does not suffer from a background due to electron recoils near the detector surface. However, the presence of three different nuclei within the crystal makes it difficult to fully characterize the crystal's response through the use of in-situ calibrations. Preliminary measurements by CRESST find no dark matter signal. 


\section{CDMS II - SUF}

The CDMS collaboration has developed another detector technology for dark matter

detection. The detectors are crystals of Ge and Si with sensors that simultaneously measure the ionization and athermal phonons produced by a recoil. These detectors are described in more detail in Ch. 2. They are capable of discrimination due to the reduced ionization for a nuclear recoil. The detectors are designed to also measure timing information from the athermal phonon signal making it possible to reconstruct the location of the interaction. The ability to reconstruct the position of an event enables the rejection of the background from electron recoils near the surface. The use of both $\mathrm{Si}$ and Ge detectors allows for an estimate of neutron backgrounds since the cross section for neutrons in Si is 5-7 times larger in Si than in Ge. The CDMS II experiment [42] observes no dark matter detection at the Stanford Underground Facility. The sensitivity was again limited by the irreducible neutron background. 


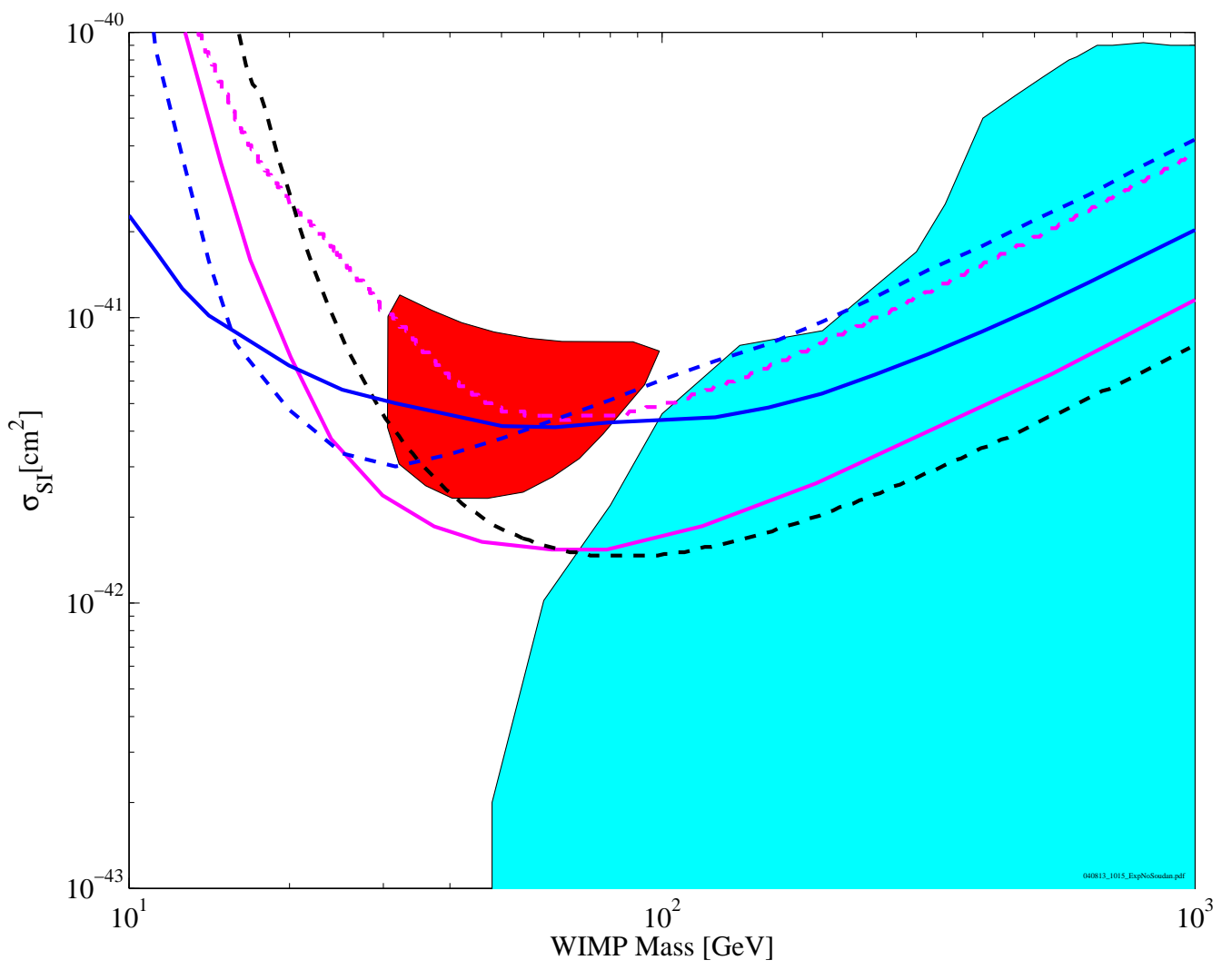

Figure 1.11: Plot of $\sigma_{S I}$ per nucleon versus WIMP mass. Exclusion curves for EDELWEISS (black dashed) [40], CDMS I - SUF (blue dashed) [41, 43], CDMS II - SUF (blue solid) $[42,37]$, ZEPLIN (magenta solid), and CRESST (magenta dashed). Cyan region corresponds to allowed models from Fig. 1.8[32]. Red region is the DAMA detection claim [39]. Limitplot courtesy of [44]. 


\section{Chapter 2}

\section{Detectors}

\subsection{The Phonon Measurement}

CDMS ZIP detectors determine the recoil energy from an interaction in the crystal by measuring the athermal phonons produced by the interaction. The phonon sensors consist of 148 cells each of which contain an array of 24 QETs (Quasiparticle assisted Electrothermal feedback Transition edge sensors) [45]. Each QET is comprised of 8 Aluminum fins connected to a thin strip of Tungsten.

\subsubsection{Phonons in the Crystal}

Phonon propagation in the crystal is dominated by two scattering processes. The first process is isotopic scattering where a phonon elastically scatters off of a $\mathrm{Ge}(\mathrm{Si})$ isotope [46]. Isotopic scattering alters the direction of phonon propagation and mixes phonon modes. The second process is anharmonic decay, where a single phonon spontaneously splits into two or more phonons [47]. Anharmonic decay reduces the average frequency of the phonons. The cross-sections for both scattering processes depend on the phonon frequency.

$$
\Gamma_{I S} \propto \nu^{-4} \text { and } \Gamma_{A D} \propto \nu^{-5}
$$

It is useful to divide phonons in the crystal as being in one of two classes. The 


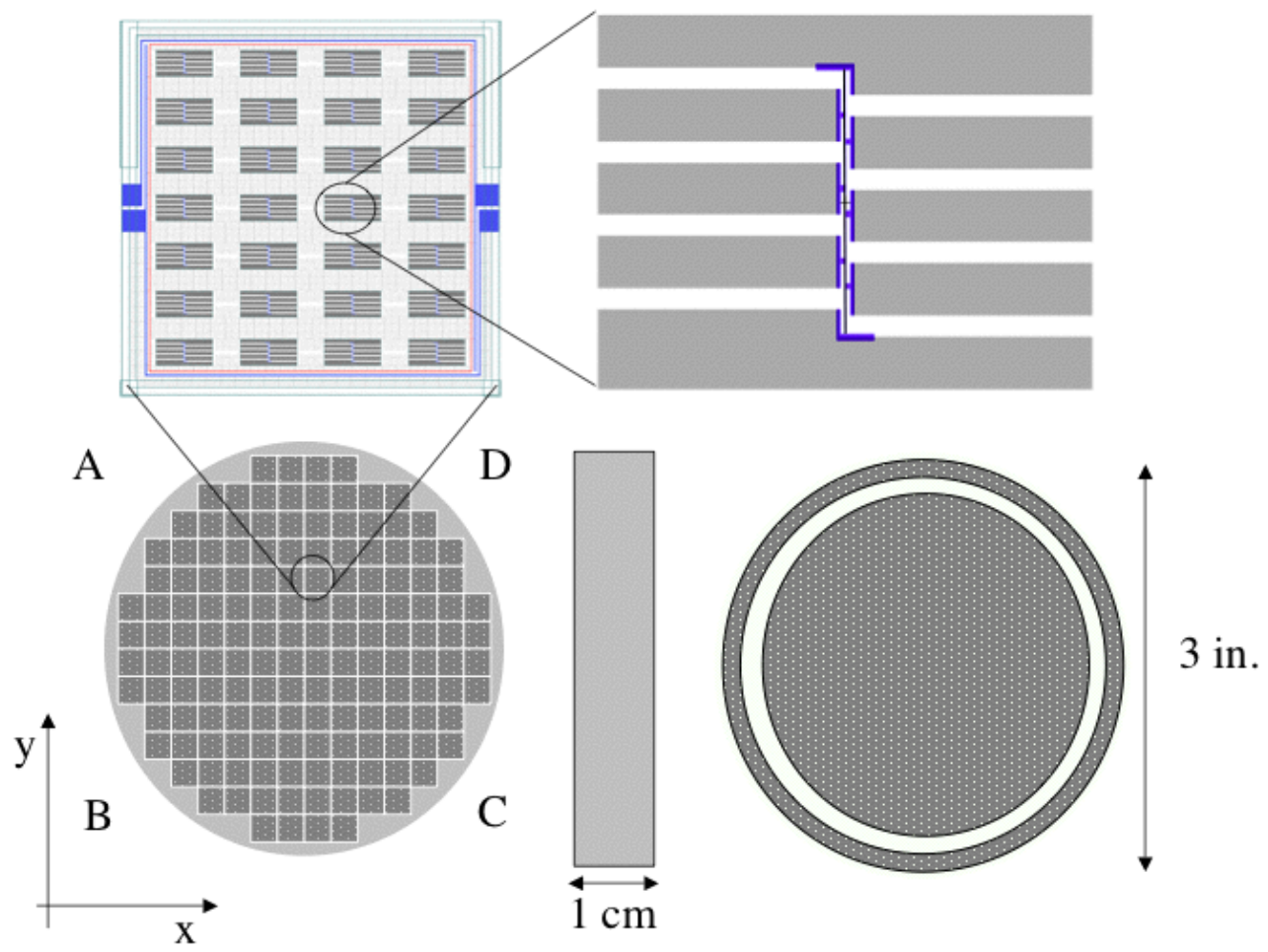

Figure 2.1: Diagram of a ZIP detector. Bottom right is the "Ionization Side" showing a large inner electrode and an outer guard ring. Bottom left is the "Phonon Side" whose surface is divided into four quadrants labeled A, B, C, and D. The axis shown defines the basis for the $\mathrm{x}-\mathrm{y}$ coordinate system. Each quadrant has 148 cells (top left) each of which have 24 QETs. A QET (top right) consists of a $1 \mu$ thick strip of W connected to $8 \mathrm{Al}$ collector fins.

first class of phonons are ballistic phonons. These phonons are sufficiently low in frequency $(0.1-1 \mathrm{THz})$ that their mean free path is comparable to the size of the crystal. These phonons will not scatter inside the crystal, but will travel in a straight line across the crystal reflecting at the boundaries until they are absorbed by metal on the surface. The second class of phonons have very high frequencies $(\sim 3 \mathrm{THz}$ for $\mathrm{Ge}$ and $\sim 6 \mathrm{THz}$ in $\mathrm{Si}$ ) and scatter frequently. The propagation resulting from the combined isotopic scattering and anharmonic decay is referred to as quasi-diffuse 
propagation [48]. For quasi-diffuse propagation, the phonon "wave front" moves at $\sim 1 / 3$ the speed of sound. The initial phonon spectrum produced by a recoil consists primarily of phonons of the second class.

There are three sources of ballistic phonons. Neganov-Luke [49, 50] phonons are produced when drifting the electric charges across the crystal to the electrodes at the surface. It has been shown that Luke phonons are ballistic and have a total energy equal to the work done in drifting the charges across the crystal [51]. The second source of ballistic phonons is electron-hole recombination at the electrodes. In reality, the phonons released when the electrons relax to the valence band are high frequency phonons. However, since these phonons are at the detector surface, they interact frequently with metal at the surface resulting in a rapid down conversion to ballistic phonons. Similarly, the third source of ballistic phonons is the interaction of the initial quasi-diffuse phonon cloud with the surface resulting in rapid down conversion into ballistic phonons [51]. This third effect is only possible if the interaction is sufficiently close to the surface of the crystal.

\subsubsection{Measuring Phonon Energy using QETs}

\section{Al fins and quasi-particle diffusion}

When the phonon wave-front encounters the Al fins at the detector surface, $\sim 30 \%$ of the phonon energy will be transferred into the Al. The Al fins are superconducting at our operating temperatures and have a superconducting energy gap of $340 \mu \mathrm{eV}$. If the energy absorbed is larger than the energy gap, the absorption results in quasiparticle (broken Cooper pairs) excitations in the Al which diffuse into the W TES. The lower band gap in $\mathrm{W}$ traps the quasi-particles in the TES so that they deposit their energy into the $\mathrm{W}$ electron system.

\section{ETF TES}

The W strips are operated as ETF TESs (Electro-Thermal Feedback Transition Edge Sensors) [52]. In this configuration, a voltage bias generates a current through the W which, through Joule heating, keeps the temperature of the W electrons somewhere 


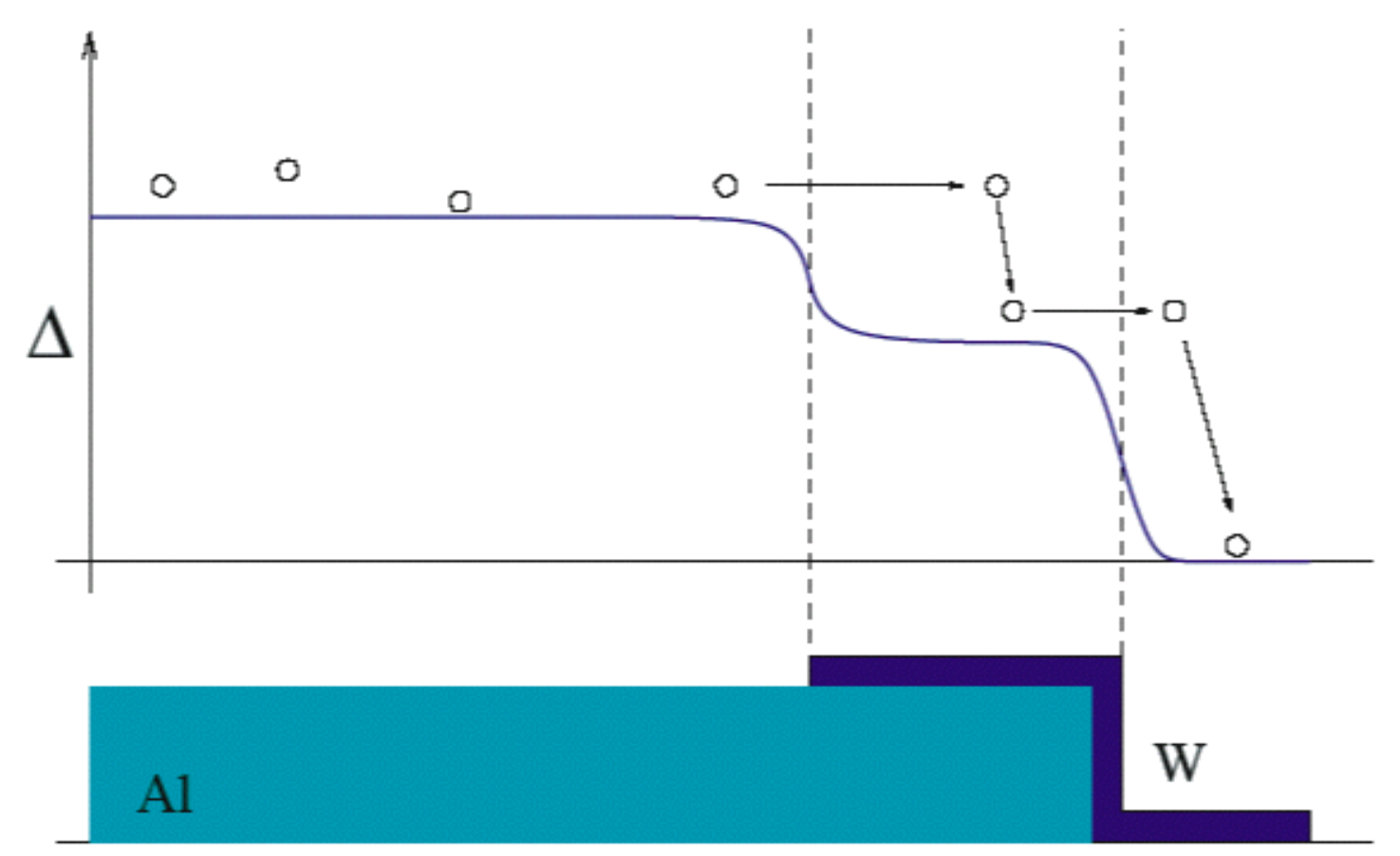

Figure 2.2: Band diagram of the Al-W overlap region. Should quasi particles in the overlap lose some of their energy, they will be unable to leave the $\mathrm{W}$ since they would be sub-gap in the $\mathrm{Al}$

in the superconducting transition. The trapped quasi-particles deposit their energy into the $\mathrm{W}$ electron system and heat it up. Since the electrons are kept in the superconducting transition edge, a small increase in the electron temperature causes a large increase in the $\mathrm{W}$ resistance. The resistance change results in a decrease in the current being supplied by the voltage bias reducing the heat and stabilizing the system.

The current response to a small change in temperature is

$$
\frac{d I}{I}=-\alpha \frac{d T}{T}
$$

where $\alpha=\frac{d R / R}{d T / T}$ is a dimensionless parameter corresponding to the slope of the W's $\mathrm{R}$ vs $\mathrm{T}$ curve. Under optimal bias conditions, $\alpha$ is usually around 100. Details of 
ETF are discussed in detail elsewhere [53, 54, 55, 37]. One particularly useful aspect of ETF is that it is a negative feedback process and therefore, stable. This means that at any time for a given input TES input power $P(t)$ we have

$$
0=\frac{d T}{d t} \propto I V-\kappa T^{5}+P(t)
$$

In Eqn. (2.3), we assume that $T \gg T_{\text {fridge }}$ and define $\kappa$ to be a constant associated with the cooling of the $\mathrm{W}$ electrons via a weak coupling to the $\mathrm{W}$ phonon system. Define

$$
I=I_{0}+\Delta I, T=T_{0}+\Delta T,
$$

where $I_{0}$ and $T_{0}$ are constant values corresponding to the situation where there is no external power.

$$
P(t)=\kappa T_{0}^{5}\left(1+\frac{\Delta T}{T_{0}}\right)^{5}-V\left(I_{0}+\Delta I\right)
$$

If we expand Eqn. (2.5) and use $0=I_{0} V-\kappa T_{0}^{5}$, we find

$$
P(t)=\kappa T_{0}^{5} \frac{\Delta T}{T_{0}}-V \Delta I
$$

Since we are operating within a very sharp superconducting transition ( $\alpha$ is large), $\Delta T / T \ll \Delta I / I_{0}$ and we get

$$
P(t)=-V \Delta I
$$

Thus, the reduction in Joule heating corresponds to the total additional power deposited into the TES.

\subsubsection{SQUIDs}

The current being supplied to the TES via the voltage bias is measured by using a SQUID (Superconducting Quantum Interference Device) [56]. Details of how SQUIDs work can be found in $[57,58]$. For our discussion, it is sufficient to view the SQUID as a device that transforms magnetic flux to voltage. The circuit shown in 2.3 outlines the current measurement. The SQUID is connected to a feedback amplifier which is locked 


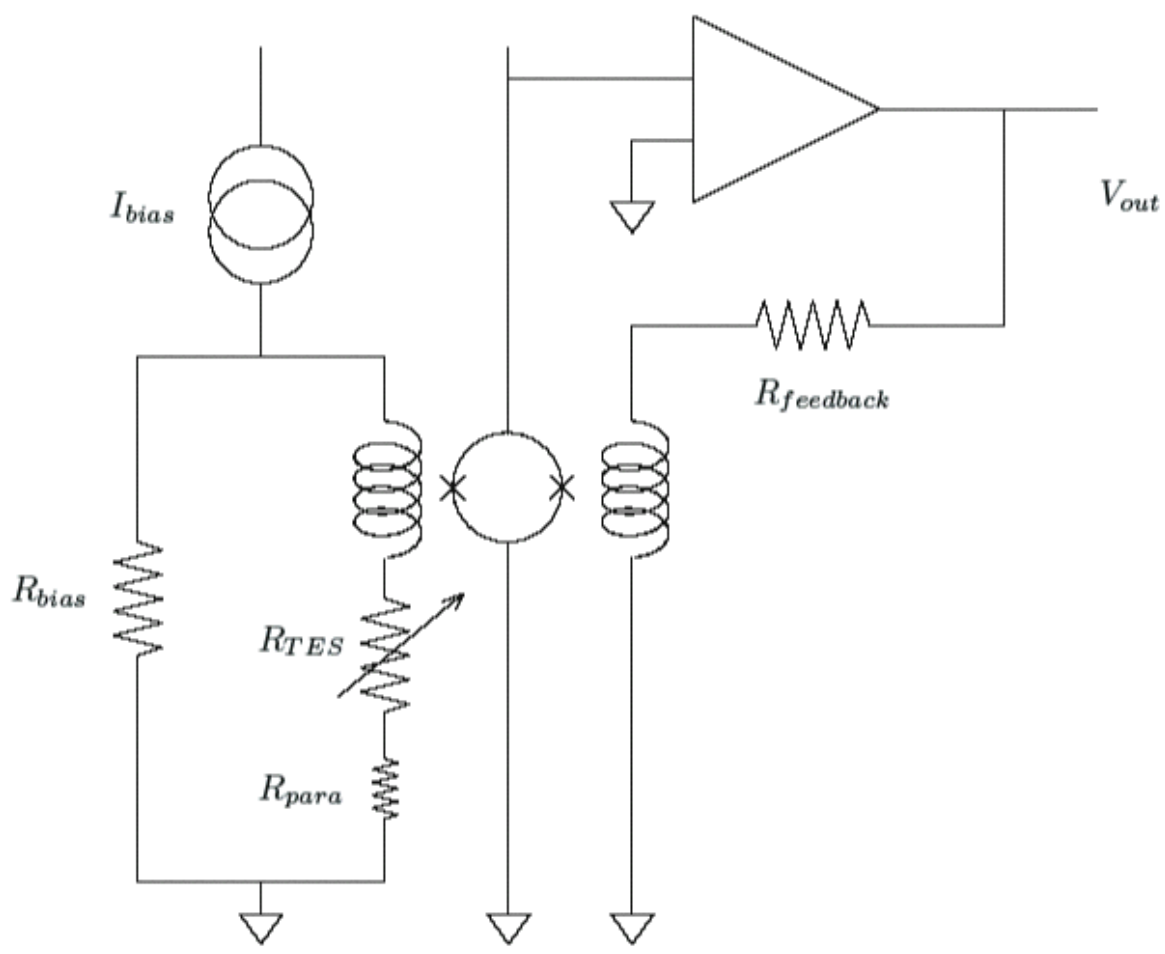

Figure 2.3: Schematic of TES current measurement circuit. The Input coil is the coil on the left. The feedback coil is the coil on the right.

to a particular voltage drop, $V_{0}$, across the SQUID. This voltage drop corresponds to a certain amount of magnetic flux inside the SQUID loop. If the current through the TES changes, the magnetic flux from the input coil also changes resulting in a change in the magnetic flux and hence the voltage across the SQUID. Once the SQUID voltage deviates from $V_{0}$, the feedback amplifier changes the current supplied to the feedback coil to return the SQUID to the original voltage. For sufficiently slow changes in the input coil current, the current supplied to the feedback coil is precisely what is required to cancel the additional magnetic flux from the input coil and we have $I_{\text {feedback }}=N I_{\text {input }}$ where $N$ is the turn ratio between the input and feedback coils. 


\subsubsection{TES Sensitivity to Bias Conditions}

It is apparent from Eqn. (2.2) that the current response is strongly dependent on the shape of the W R vs T curve and the TES bias point within the curve. An important situation arises when the power input is sufficiently large to drive the TES out of the transition. Once the TES is in the normal state, $\alpha$ becomes nearly zero and there is very little change in current. A TES in such a state is considered "saturated." Fig. 2.4 displays the trace for a saturating TES. For a saturated TES, the total integral of the current change remains unaffected and is still equal to the total energy deposited. However, saturation results in a change in the pulse shape and gives an upper bound to the possible current change of a given TES. This limitation is important since it restricts the current output for TESs that are biased high in their transition. In order to minimize saturation effects, the TES bias needs to be minimized. ${ }^{1}$

The coil shown in Fig. 2.3 couples the TESs to the SQUID. The effects of the inductance of the input coil cannot be neglected. The configuration is essentially a low pass filter which begins to roll off at a frequency $\sim \frac{R}{2 \pi L}$ where $L$ is the inductance of the input coil and $R$ is the resistance of the TES. The inductance of the input coil is $\sim 0.25 \mu \mathrm{H}$, so for a TES biased to a resistance of $\sim 0.25 \mathrm{Ohm}$, the corresponding roll off is $\sim 160 \mathrm{kHz}$ as shown in Fig. 2.5. This roll off means that the input coil begins to filter away changes with timescales $\sim 1 \mu \mathrm{s}$. Biasing a TES lower will result in filtering signals that are on timescales comparable to that of the phonon starttimes.

\footnotetext{
${ }^{1}$ The TESs cannot be biased too low or it will go into electro-thermal oscillations as described in $[53,54]$
} 


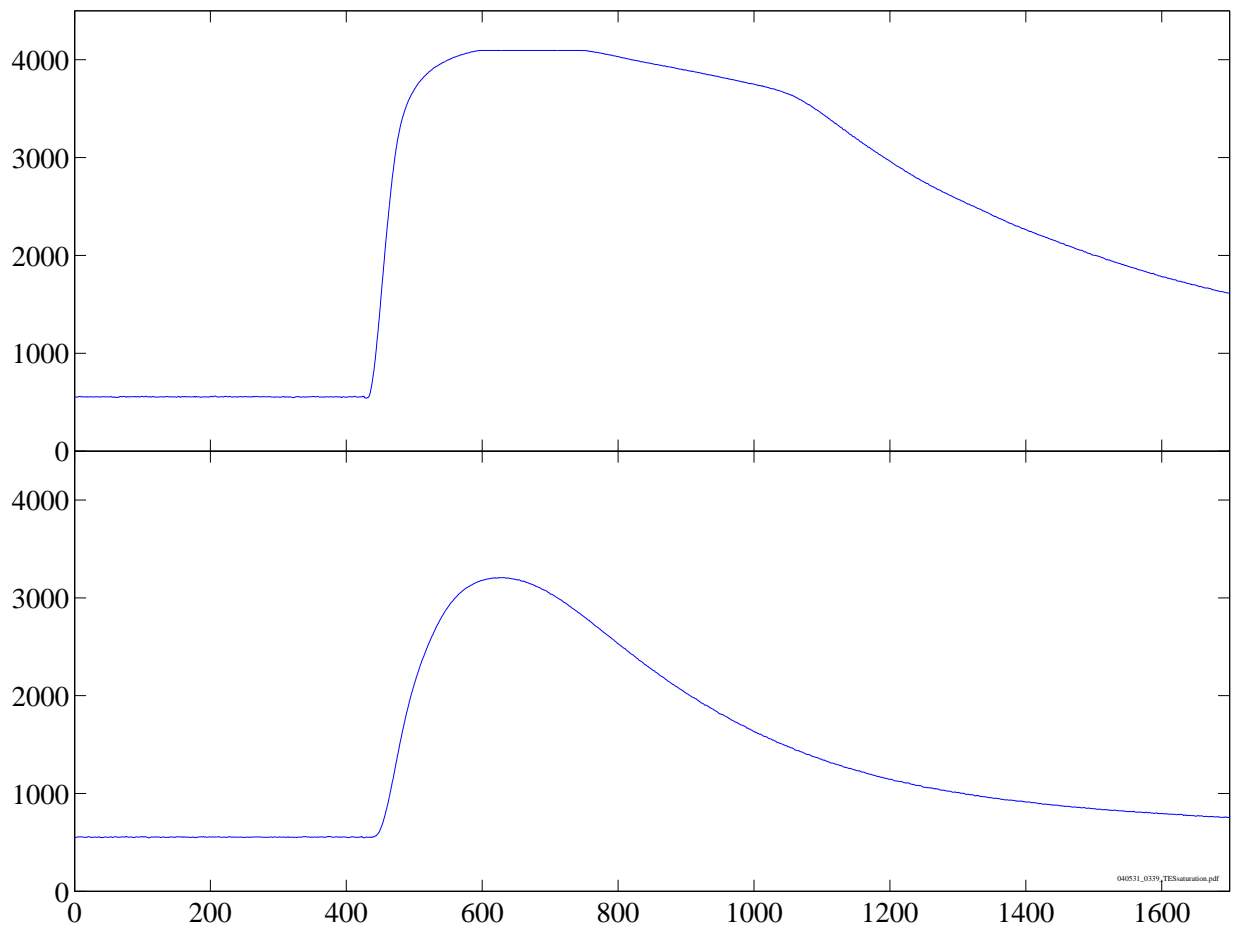

Figure 2.4: A saturated (top) and unsaturated (bottom) TES trace showing the change in pulse shape due to TES saturation. In both cases, the total area corresponds to the energy of the pulse. 


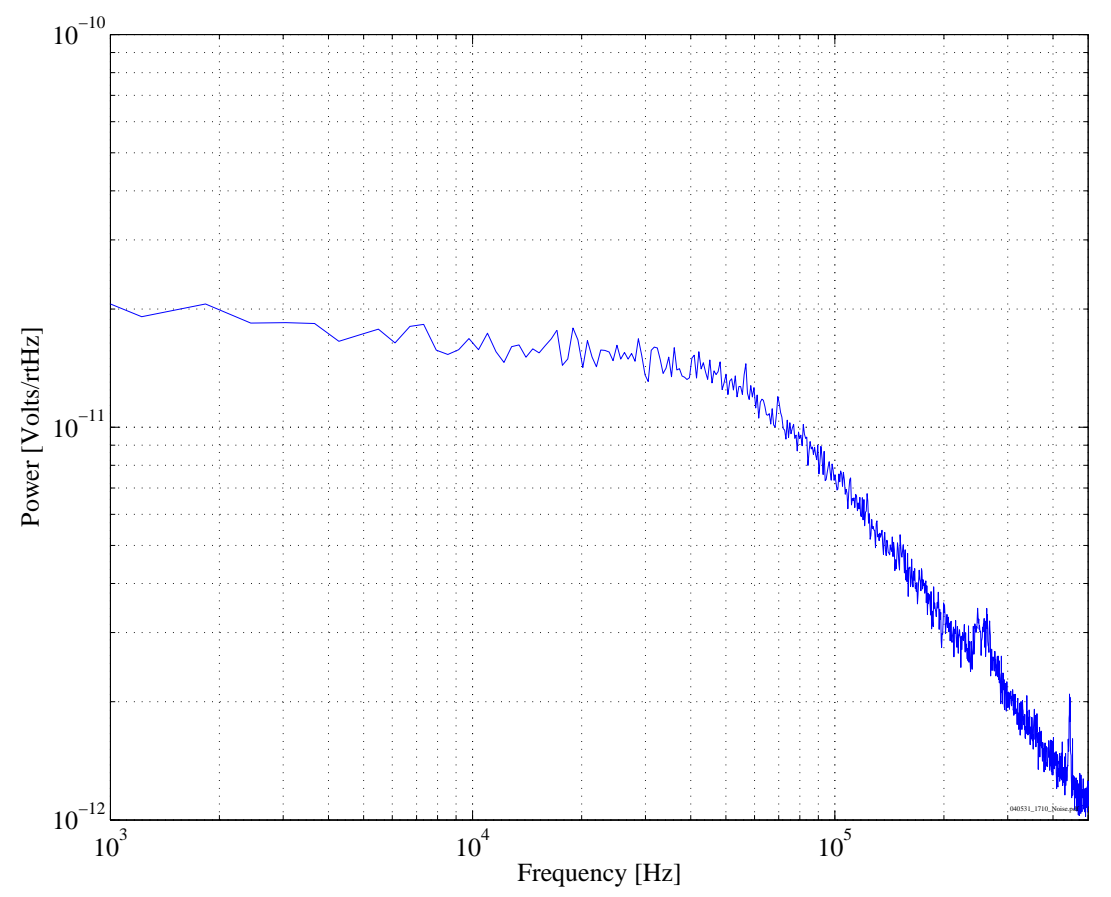

Figure 2.5: Noise power spectrum of a biased TES showing the L/R roll off at 160 $\mathrm{kHz}$ from the SQUID input coil.

\subsection{The Ionization Measurement}

\subsubsection{Measuring the Ionziation}

The circuit outlined in Fig. 2.6 measures the ionization due to a recoiling electron or nucleus. A voltage applied across the detector creates an electric field that drifts the electrons and holes in the lattice to electrodes on the surface of the detector. The result is that a current flows through the detector. The electrodes are connected to a low noise current integrator.

The current due to the drifiting of the electron-hole pairs is given by

$$
I_{o}=\sum_{\text {all pairs }} \frac{e}{d}\left(v_{\text {electron }}+v_{\text {hole }}\right)
$$




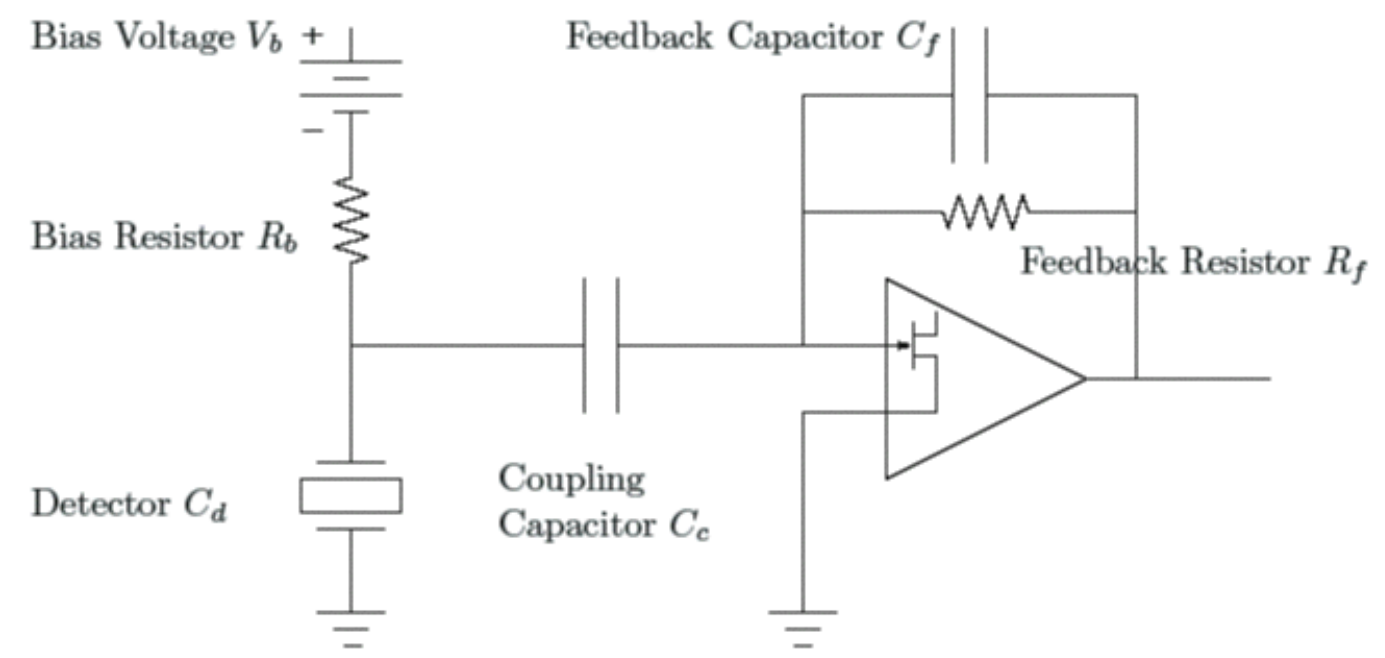

Figure 2.6: Ionization circuit schematic

Where $v_{\text {electoron (hole) }}$ is the constant drift velocity across the crystal and $d$ is the thickness of the crystal. In the case where the electron and hole drift to their corresponding electrodes, we have $\int\left(v_{e}+v_{h}\right) d t=d$. Thus, integrating the current gives us the total charge drifted across the crystal.

In reality, the charge on the feedback capacitor is only proportional to the total ionization since the detector itself has a capacitance. The current seen by the coupling capacitor is

$$
I=\frac{C_{c}}{C_{c}+C_{d}} I_{o}
$$

where $C_{c}$ is the coupling capacitance $(1 \mathrm{pF})$ and $C_{d}$ is the detector capacitance (300 $\mathrm{nF})$.

\subsubsection{Poor Charge Collection}

Since the charge on the feedback capacitor is proportional to the charge drifted across the detector, if either the electron or hole fail to drift completely across the detector, 


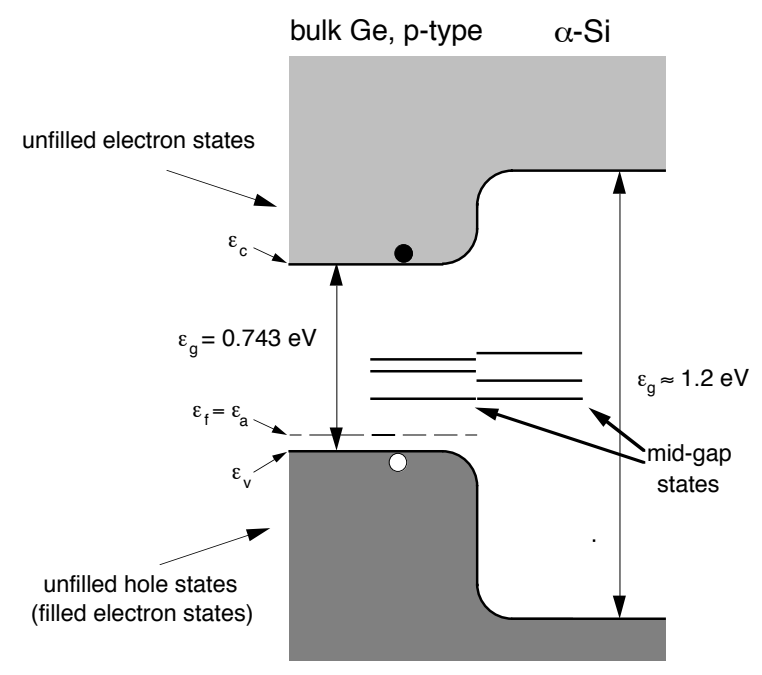

Figure 2.7: Schematic of the band structure at the Ge- $\alpha$-Si interface. The $\alpha$-Si creates a small potential barrier for both electrons and holes making it more difficult for charges to drift into the wrong electrode. Mid-gap states that may define the alignment of the gaps are also shown. Figure from [43].

the integrated current would be reduced, since $\int\left(v_{e}+v_{h}\right) d t<d$, and would underestimate the real ionization. There are two mechanisms that yield incomplete charge drift: charged impurities and the dead layer.

Charged impurities in the crystal can trap drifting electrons and holes and prevent them from reaching their intended electrodes. By converting charged impurities to neutral impurities, trapping can be reduced by a few orders of magnitude. The neutralization of the charged impurities is accomplished by using LEDs to illuminate the detector with photons that are sufficiently energetic to create electron-hole pairs. In the absence of a voltage bias, the created electron-hole pairs either recombine, or are trapped by charged impurities. As long as the detector temperature is sufficiently low, charges trapped by the impurity remain on the impurity and screen the impurity's charge. Over a sufficiently long period of time, the charge accumulated on the impurity is sufficient to completely cancel the impurity's intrinsic charge renderring it neutral.

The dead layer [59] of a detector refers to a region of the detector in which charges 
can diffuse into the incorrect electrode. The existence of such a layer is especially problematic for CDMS since beta particles do not penetrated deeper than 10 microns. The depth of the dead layer can be reduced to a few microns by depositing a thin layer of amorphous $\mathrm{Si}(\alpha-\mathrm{Si})$ between the crystal and the electrodes [60]. The $\alpha$-Si provides a barrier against charge diffusion into the wrong electrodes as seen in Fig. 2.7. The application of a drift field enables the correct charges to overcome this barrier. 


\subsubsection{Quenching and Discrimination}

The ionization measurement can be combined with a measurement of the recoil energy to determine whether the recoiling particle was an electron or a nucleus. The basis for this discrimination is a phenomenon referred to as "quenching." Simply put, "quenching" is the fact that electrons and nuclei produce different amounts of ionization as they pass through the crystal. In general, recoiling electrons produce more ionization. The "quenching factor" is just the ratio of the ionization from a recoiling nucleus to the ionization produced by a recoiling electron of the same recoil energy.

Disitinguishing between electron and nuclear recoils is the primary method CDMS employs to reject backgrounds. Most sources of backgrounds will scatter off of the electrons in the crystal whereas a WIMP will scatter off the nuclei. The consequence of the dead-layer should now be apparent. Electron recoils in the dead-layer have deficient charge collection and can be mistaken as nuclear recoils. In particular, external electrons (from now on referred to as "betas" to distinguish them from "electron recoils" where we are referrring to the fact that the recoiling particle is an electron) will scatter only within the dead layer and thus constitute a significant background for the experiment.

\subsection{Event Discrimination}

The power of the ZIP detectors lies in their ability to reconstruct the nature of an interaction due to the scattering from an external particle. By identifying the nature of the recoil, it is possible to reject virtually all interactions that are non-WIMP induced. The primary means of background rejection is discrimination between nuclear and electron recoils based on the "quenching" of the ionization for nuclear recoils described in Sec. 2.2.3. We define the "ionization yield" to be the ratio of ionization (in keVee ${ }^{2}$ ) to the recoil energy. By construction, electron recoils will have yields 1. Nuclear recoils, which have reduced ionization, have yields $\sim 0.3$.

\footnotetext{
${ }^{2} 1 \mathrm{keVee}$ (ee stands for electron equivalent) corresponds to the charge produced by a $1 \mathrm{keV}$ photon.
} 


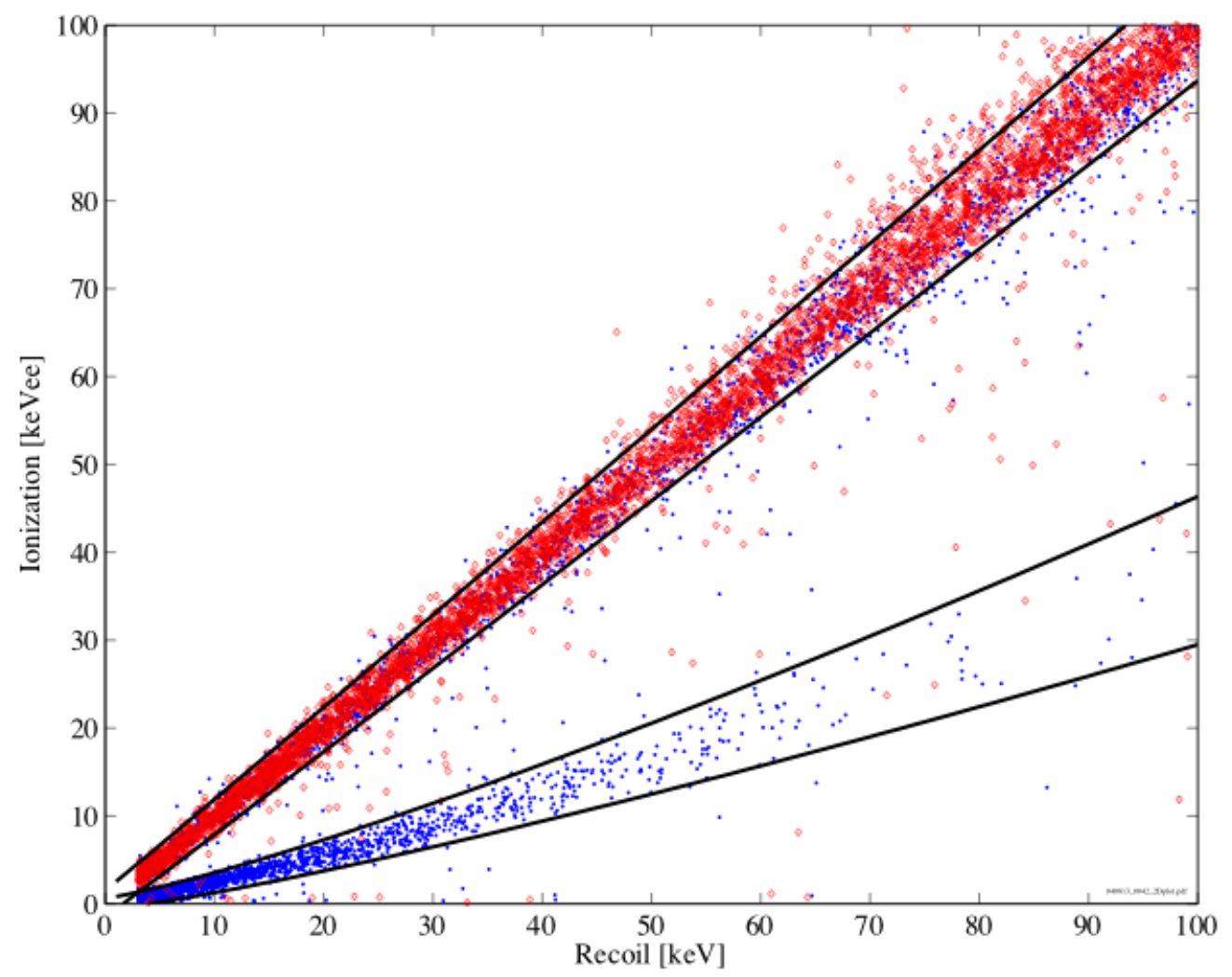

Figure 2.8: A plot of ionization versus recoil for a gamma $\left({ }^{133} \mathrm{Ba}\right)$ and neutron $\left({ }^{252} \mathrm{Cf}\right)$ calibration. The gamma calibration is red. The neutron calibration is blue. There are two distinct populations corresponding to electron recoils and nuclear recoils.

Backgrounds for the experiment fall into one of three categories :

- non-WIMP induced nuclear recoils

- electron recoils in the bulk

- electron recoils in the dead-layer

Bulk electron recoils are rejected by the ionization yield parameter alone. Electron recoils in the dead layer are rejected by a combination of a cut on the ionization 
yield parameter and a cut on the phonon pulse shape. Non-WIMP induced single scatter nuclear recoils are due to neutrons and cannot be rejected on an event by event basis. However, neutrons multiply scatter, whereas WIMPs do not. Additionally, the dependence of the neutron cross-section on target material is different from that of a WIMP. Monte Carlo simulations use the number of multiple scatters and the nuclear recoil rate in $\mathrm{Si}$ to provide estimates for the contamination of our WIMP signal from a neutron background.

\subsubsection{Non-WIMP nuclear recoils - "Neutrons"}

Neutrons comprise the only background that cannot be rejected on an event by event basis. However, a neutron signal differs from a WIMP signal due to two facts: neutrons will multiply scatter and neutrons are more likely to scatter in Si than WIMPs. The expected spectrum from neutrons has to be determined from Monte Carlo. The neutron simulations have been developed sufficiently so that during Run 19 (CDMSI) and Run 21 (CDMS II) a background subtraction of veto-anticoincident cosmogenic neutrons could be performed. The neutron simulations for the first run at Soudan predict a negligible contamination from unvetoed cosmogenic neutrons.

\subsubsection{Bulk Electron Recoils - "Gammas"}

The majority of backgrounds are rejected on an event by event basis by a cut on the ionization yield parameter. The cut is implemented as a definition of two bands: an electron recoil band and a nuclear recoil band, as shown in Fig. 2.9. Only events in the nuclear recoil band are accepted. The bands themselves are defined from calibrations with external photon $\left({ }^{133} \mathrm{Ba}\right)$ sources and neutron $\left({ }^{252} \mathrm{Cf}\right)$ sources. The parameterization of the bands is described in [43], and is of the form $Y_{0} \pm 2 \sigma$, where

$$
\begin{gathered}
Y_{0}\left(E_{R}\right)=\left\{\begin{array}{cc}
a E_{R}^{b} / E_{R}, & \text { for Nuclear Recoils } \\
e+a E_{R}+b E_{R}^{2} & \text { for Electron Recoils }
\end{array}\right. \\
\sigma\left(E_{R}\right)=\left[c Y_{0}\left(E_{R}\right) E_{\text {recoil }}+d\right] / E_{R}
\end{gathered}
$$




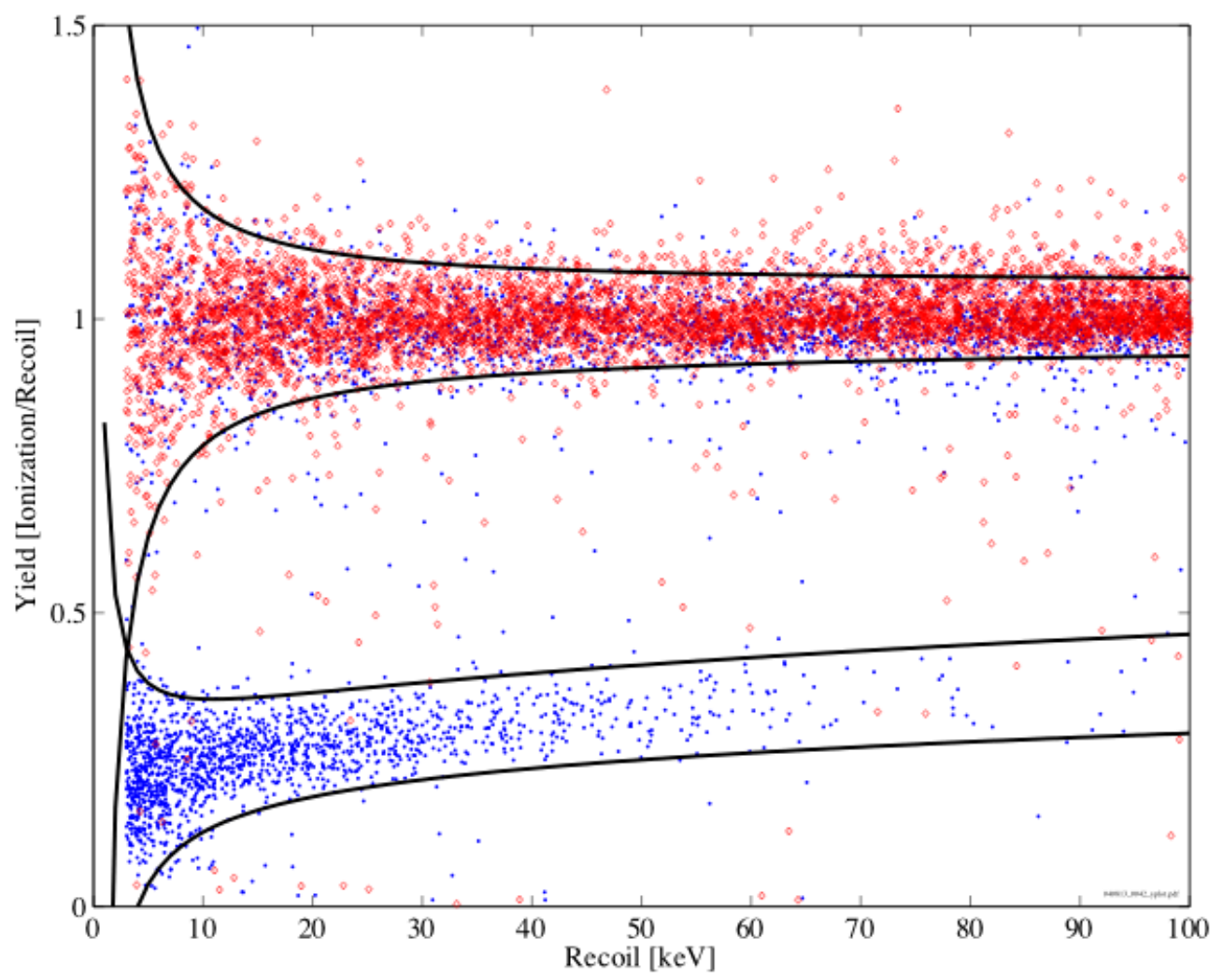

Figure 2.9: Plot of the ionization yield parameter (ionization/recoil) for a gamma $\left({ }^{133} \mathrm{Ba}\right)$ and neutron $\left({ }^{252} \mathrm{Cf}\right)$ calibration showing the electron $(y \sim 1)$ and nuclear $(y \sim 0.3)$ recoil bands. The gamma calibration is in red and the neutron calibration is in blue.

where $E_{R}$ is the recoil energy. The parameters $a, b, c, d$, and $e$ are determined empirically from the calibration data. For the nuclear recoil band, we divide the neutrons from the neutron calibrations into a few recoil bins. In each bin, we fit the yield distribution to a gaussian. We then fit the means with $Y_{0}$ to determine $a$ and $b$ and we fit the widths to $\sigma$ to determine $c$ and $d$. A similar procedure using photons from the gamma calibrations determines the parameters for the Electron Recoil band. 


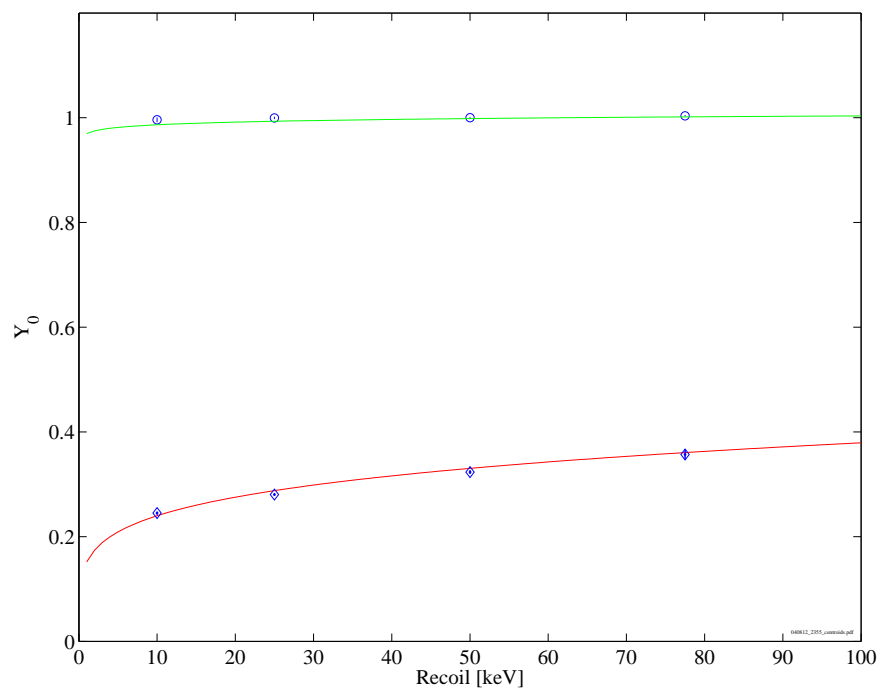

Figure 2.10: Plot showing fit of the band centroids, $Y_{0}\left(E_{R}\right)$, to the means of the yield distributions for neutrons (diamonds) and gammas (circles).
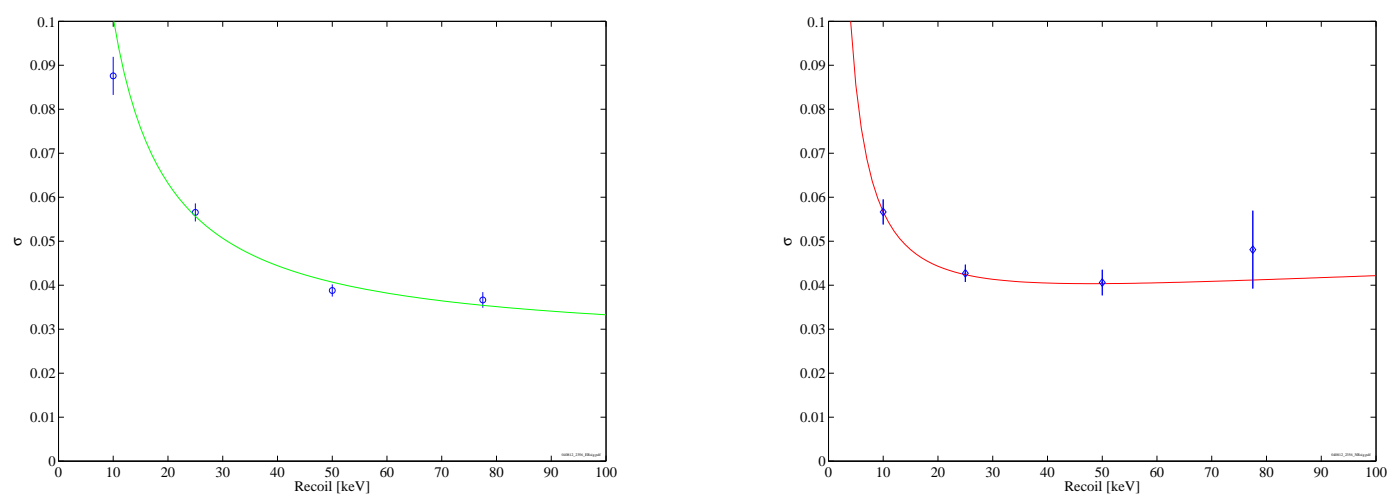

Figure 2.11: Plots showing the fits of the widths, $\sigma\left(E_{R}\right)$, to the standard deviation of the yield distributions for gammas (left) and neutrons (right). 


\subsubsection{Surface Electron Recoils - "Betas"}

As described earlier, events occuring close to the surface of a detector have poor charge collection and are difficult to reject based on their ionization yield alone. In order to reject electron recoils near the surface, we need to utilize additional information from the phonon pulse shape. Specifically, the risetime of the phonon pulse can be used to discriminate between surface and bulk events [51,61]. Events close to the surface of the crystal typically have faster risetimes than events in the bulk. The reason for this effect is that near the surface, the initial high frequency phonons interact with the metal on the surface resulting in a more rapid down conversion to ballistic phonons which propagate to the QETs sooner.

It turns out that the cuts on phonon timing parameters can be determined from gamma calibrations. It is possible that when high energy x-rays scatter in material near the detector an electron is "ejected" from the material and hits the detectors. Such an event is referred to as an "ejectron." Since the "ejectron" is an electron, it is not deeply penetrating. During gamma calibrations, about $1 \%$ of the events in a given detector are actually "ejectrons."

We use ejectrons and neutrons to determine cuts for two phonon timing parameters: the start time of the primary channel and the $10 \%-40 \%$ risetime of the primary channel. First the data is divided into separate energy bins. In each energy bin, a cut value is determined by maximizing the fraction of neutrons passing the cut while minimizing the Poisson error on the fraction of ejectrons passing the cut [61, 62]. These cut values are then fit to a functional form. It is worth noting that the low statistics from the ejectrons yield a great deal of variability in where the timing cuts can be set. For this analysis, this cut was set with no prior knowledge of the event distribution in the WIMP search data to guarantee that the cut is set in an unbiased manner. The exact parameterization of the cut is detailed in [63].

Fig. 2.12 shows how a cut in phonon timing rejects all ejectrons while accepting nearly $80 \%$ of the neutrons. The phonon timing cut rejects bulk electron recoils as well. The reason that bulk electron recoils are also rejected is that the phonon signal for electron recoils is faster than for nuclear recoils due to the larger fraction of Luke phonons. 


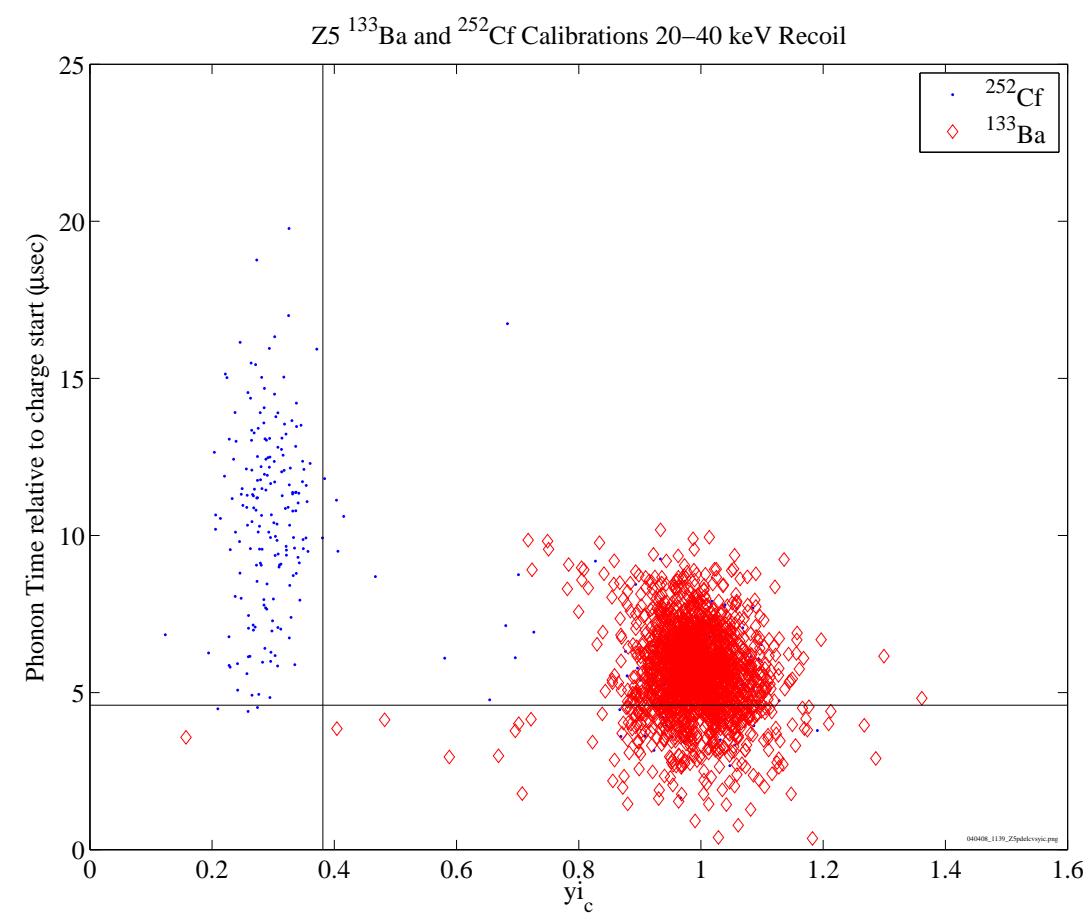

Figure 2.12: A plot showing the use of phonon pulse shape to discriminate between surface electron recoils with poor charge collection and bulk nuclear recoils. 


\section{Chapter 3}

\section{Event Reconstruction}

\subsection{Reconstructing the Interaction Location}

\subsubsection{Parameters used for the Reconstruction}

The ZIP detectors provide two kinds of information that can be used to reconstruct the location of the interaction within the crystal.

The first kind of information comes from the relative difference in the timing parameters of the phonon pulse shape. For each channel of the detector, the start time of the phonon pulse is defined to be the time that the pulse reaches $20 \%$ of its amplitude. The two coordinates of the $\mathrm{x}-\mathrm{y}$ delay are defined as the difference between the start times of the two neighboring channels relative to the start time of the primary channel. For example, for an event in channel A, the x-delay is the difference in start time between channel $\mathrm{A}$ and channel $\mathrm{D}$ and the $\mathrm{y}$-delay is the difference in start time between channel A and channel B. Fig. 3.1 shows a delay plot demonstrating the usefulness of the relative delays in reconstructing the $\mathrm{x}$-y location of each event.

The second kind of information comes from the relative energy distribution between the phonon channels. The x-partition coordinate is defined as $(P C+P D-$ $P A-P B) / P T$ and the y-partition coordinate as $(P A+P B-P C-P D) / P T$. Fig. 3.2 shows a partition plot (also referred to as a "box plot"). The usefulness of these pa- 

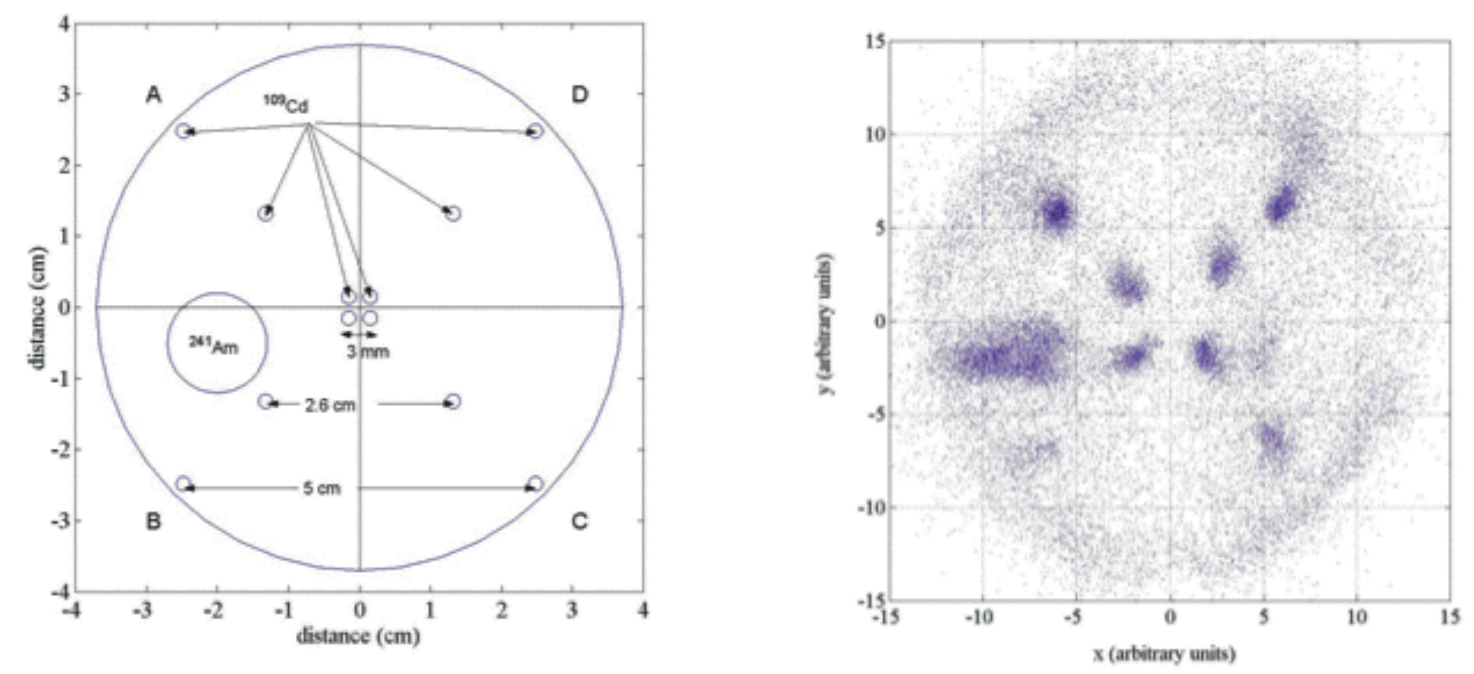

Figure 3.1: Delay plot (right) of the source configuration shown (left). The sources are a ${ }^{241} \mathrm{Am}$ (large source between channels A and B) and a collimated ${ }^{109} \mathrm{Cd}$ (small dots). The non-circular shape of the source "blobs" indicates the loss of resolution along the radial coordinate. The increased density of points near the edge indicates that the radial coordinate is piling up or even folding back.

rameters in determining the event location is demonstrated in Fig. 3.3 where the events from the highlighted ${ }^{109} \mathrm{Cd}$ blobs in Fig. 3.2 are highlighted. As expected, the particles from the ${ }^{109} \mathrm{Cd}$ source are almost all betas.

\subsubsection{The 3-dimensional Position Coordinate}

It turns out that neither of the two parameters are perfect for reconstructing the event location because both parameters are double valued. Fig. 3.4 shows both the box plot and delay plot with values in the outer electrode highlighted to illustrate the fold back of both coordinates.

It is possible to construct a monotonic event location parameter using a combination of the delays and partition. For the lookup table, we use a three dimensional parameter consisting of the two box plot parameters and a normalized radius from the delay. The delay radius is defined as $\sqrt{\mathrm{x}-\text { delay }^{2}+\mathrm{y} \text {-delay }}{ }^{2}$ and is normalized to 


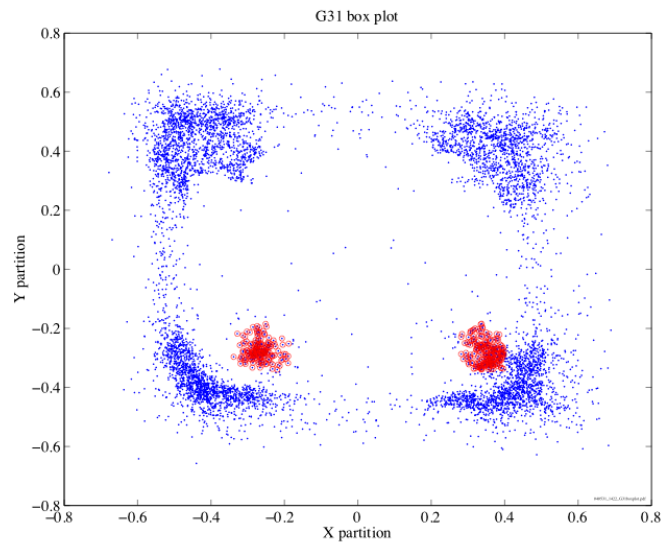

Figure 3.2: Box plot for $\mathrm{G} 31$ with the ${ }^{109} \mathrm{Cd}$ from Fig. 3.1. The 2 inner collimator holes in channels $\mathrm{B}$ and $\mathrm{C}$ are highlighted. The definition of the phonon partition coordinates yields a square shape.

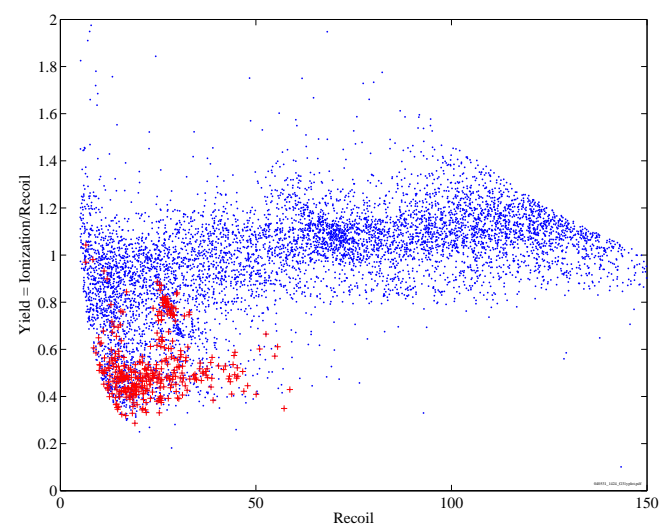

Figure 3.3: Yield vs recoil plot for events shown in Fig. 3.2. The indicated points from the ${ }^{109} \mathrm{Cd}$ blobs have reduced charge collection.

a maximum of $\sim 0.5$ so that it is on equal footing with the box plot coordinates. Fig. 3.5 shows that the true $\mathrm{x}-\mathrm{y}$ position of any event is mapped uniquely on to a two dimensional manifold in this three dimensional space. Though this mapping is monotonic, it is not uniform. 

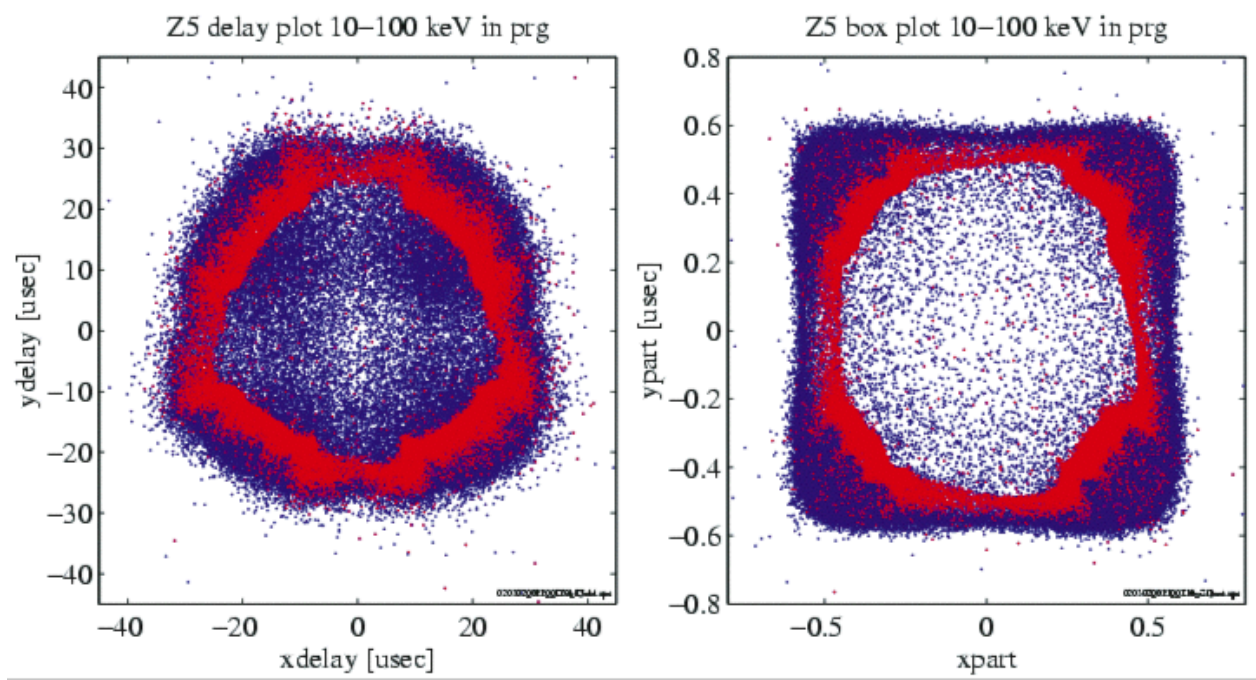

Figure 3.4: Delay plot (left) and box plot (top right). Events in the outer electrode guard ring are highlighted in red prominently showing that both location parameters are double valued.

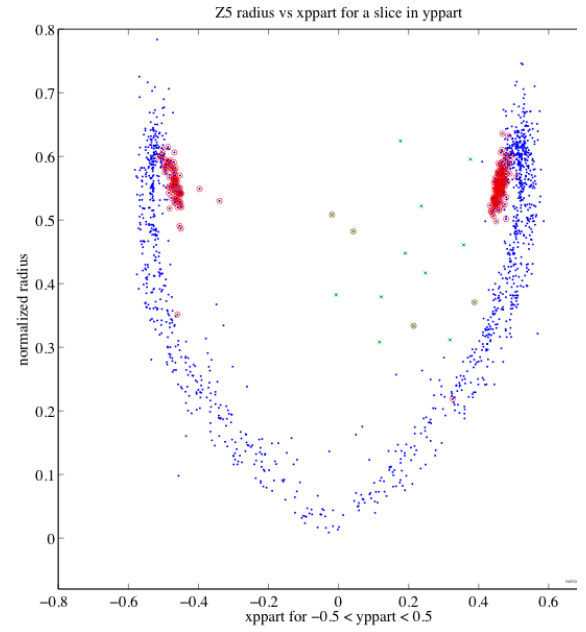

Figure 3.5: A plot for Z5 gammas of the 2D position manifold in the 3D space of xppart, yppart, and normalized radius for a slice in yppart. Events marked by red circles are towards the edge of the detector because they have substantial energy in the outer electrode. Points off of the manifold marked by green x's are most likely internal multiple scatters. 


\subsection{Position Dependence of the Detector Response}

\subsubsection{Risetime Discrimination and Position Variation}

The calibration of $\mathrm{G} 31^{1}$ is useful in demonstrating the fast risetime effect expected from events interacting near the detector surface. Fig. 3.6 shows a histogram of the total phonon risetime for events in quadrant $\mathrm{B}$. There is a clear distribution of events at faster risetime as well as a fast risetime tail from low energy gammas for which charge collection is complete. Fig. 3.6 compares the risetime distributions for events in channel $\mathrm{B}$ with events in channel $\mathrm{C}$ showing that the risetime varies with position.

Calibration data of other detectors further demonstrate position dependence of the phonon response. Figs. 3.7 and 3.8 show that both the phonon pulse amplitude and timing parameters vary across the entire surface of the detector.

It is clear from the G31 calibration data that the risetime of the phonon pulse is effective for rejecting events near the surface of the detector. However, the variation of the phonon signal across the detector surface makes it difficult to define a single rejection parameter for the entire detector.

\footnotetext{
${ }^{1}$ The calibration of G31 at UCB illuminated a detector with an external photon $\left({ }^{60} \mathrm{Co}\right)$ and neutron $\left({ }^{252} \mathrm{Cf}\right)$ source as well as an internal collimated beta $\left({ }^{109} \mathrm{Cd}\right)$ source. Data was obtained with the collimated source on either side of the detector and with multiple charge bias voltages.
} 

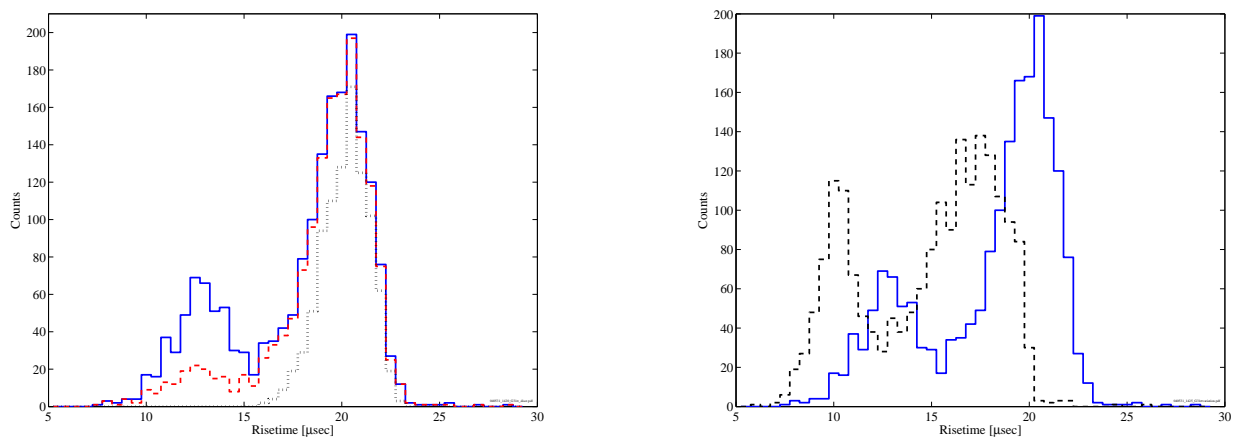

Figure 3.6: Left: Risetime distributions for channel B for all events (solid), y > 0.7 (dashed), and high energy gammas (dotted). Note that low energy gammas with full charge collection ( $\mathrm{y}>0.7$ ) have a fast risetime tail. Right: Histograms of risetime distributions for channel B (solid) and channel C (dashed). 


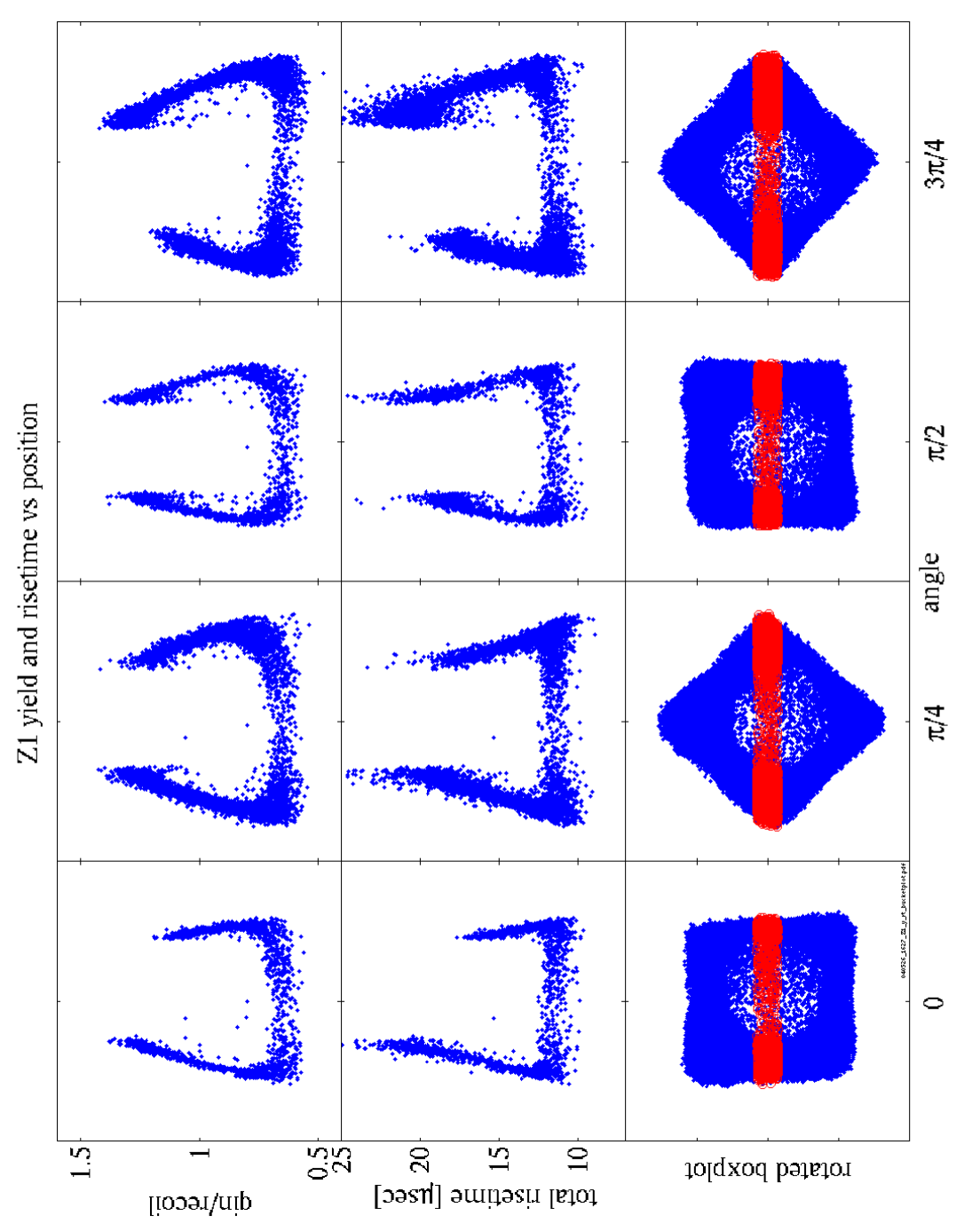

Figure 3.7: Landscape plot of yield (qsum/recoil) and total phonon risetime for Z1. Each column corresponds to a rotation of the boxplot with the indicated slice as the horizontal coordinate for the plots above the box plots. Z1 has the largest signal variation of all the detectors. It is known to have the largest $T_{c}$ gradient. 


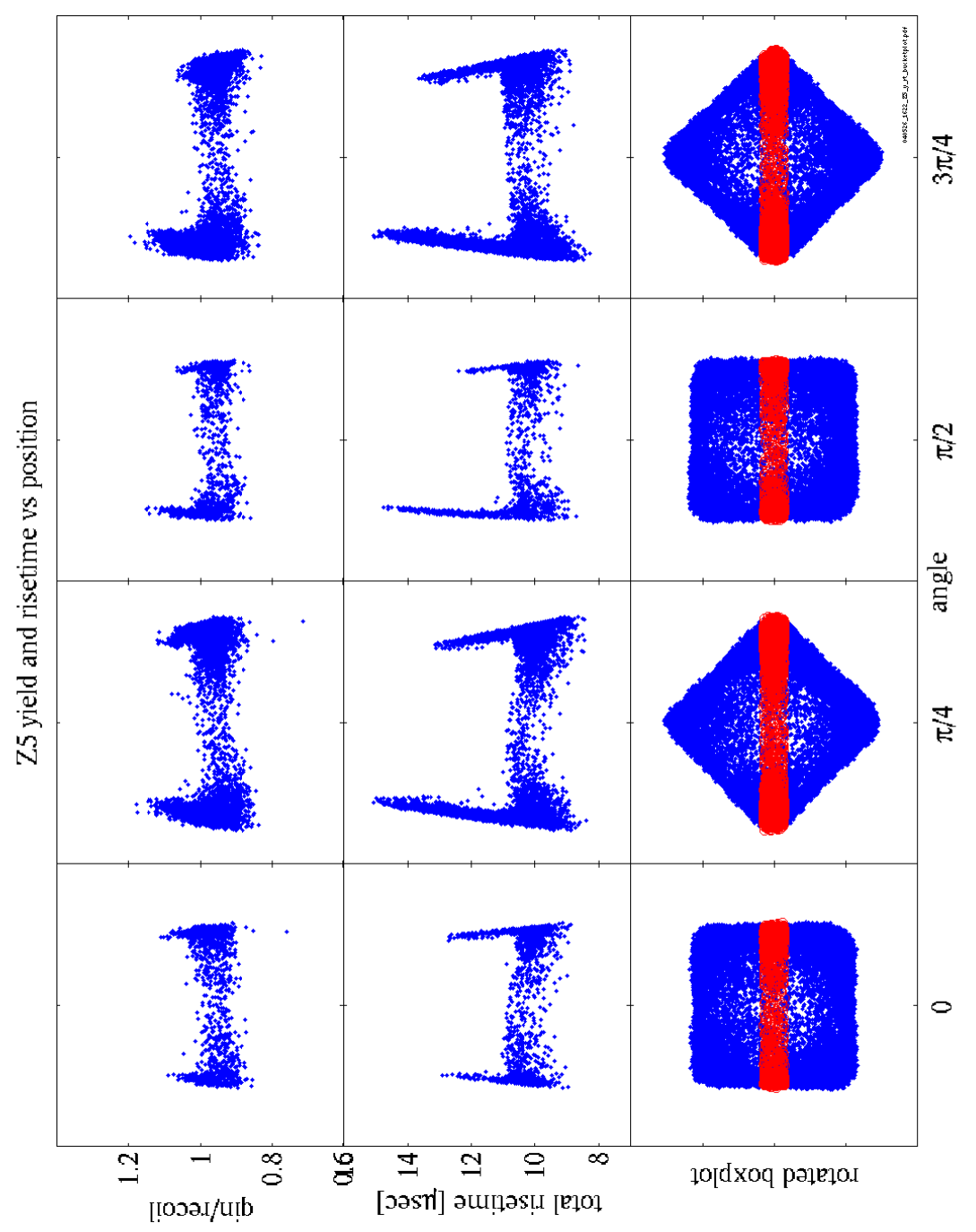

Figure 3.8: Landscape plot of yield (qsum/recoil) and total phonon risetime for Z1. Each column corresponds to a rotation of the boxplot with the indicated slice as the horizontal coordinate for the plots above the box plots. The extremely small $T_{c}$ gradient of Z5 suggests that the pulse shape variation has a component that is not related to $T_{c}$. 


\subsection{2 $T_{c}$ Gradients}

The first effect responsible for the dependence of the phonon signal on position is the existence of a gradient in the $T_{c}$ of the TESs across the surface of the detector. It is difficult to fabricate detectors with TESs that all have the same $T_{c}$. The majority of detectors initially have large $T_{c}$ gradients. Variation of the $T_{c}$ of the TESs results in a substantial dependence of the phonon pulse shape on the location of the event. The reason is obvious, since we apply a single bias for each channel, the $T_{c}$ variation of each individual TES results in the TESs being biased at a different points in their respective $R$ vs $T$ curves. As a consequence, events that are close to TESs that are biased high will have different pulse shapes from those that are near TESs that are biased low since the TESs that are biased high will saturate with less energy as well as roll off at different frequencies. Currently, the $T_{c} \mathrm{~s}$ can be tuned by strategically using ion implantation [64] to make a more uniform $T_{c}$ distribution thereby reducing the position variation. However, detectors that were commissioned prior to the development of the ion implantation strategies (like Z1) or for which the ion implantation was not ideal will have large effects due to the $T_{c}$ variation. The $T_{c}$ variation combined with the effects discussed in Sec. 2.1.4 make it impossible to obtain a detector configuration where all of the TESs on a detector have the same amplitude and frequency response.

\subsubsection{Intrinsic variation of the phonon signal and degenerate pulse shape analysis}

The second cause of position dependence of the phonon response is the dependence of the actual phonon spectrum on the real event location. Fig. 3.8 shows the variation of various phonon pulse shape parameters with respect to the box plot for a detector with a very small gradient. The extremely small $T_{c}$ gradient for this detector suggests that the residual position dependence is actually due to intrinsic phonon physics and our pulse shape analysis algorithims.

The radial variation of the detected phonon signal explains why the box plot folds back. For this discussion, it is useful to divide the absorbed phonons into two 
populations. We can define initial phonons to be phonons that deposit energy into the QETs without reflecting off of any surface. Secondary phonons will correspond to phonons that deposit their energy after reflecting at a surface. The secondary phonons are very weakly correlated to the initial event location, therefore, their energy will tend to be equally distributed among the four channels. To illustrate the fold back, we consider three cases of events:

- Events close to the center of a quadrant: For these events, the primary phonons illuminate a large number of QETs of the primary quadrant. As a result, the energy in the primary quadrant is larger than the other 3 quadrants.

- Events close to the boundaries between quadrants: For these events, the primary phonons illuminate the QETs of a number of quandrants distributing the primary energy between the quadrants. The fraction shared will reflect the fraction of QETs illuminated in each quadrant. In particular, events near the center of the detector will have the phonon energy distributed equally among the four channels.

- Events towards the edge of the quadrants: For these events, fewer QETs of the primary quandrant are illuminated by the primary phonons. As a result most of the energy is absorbed as secondary phonons leading to a more uniform distribution of energy.

The fold back of the delay plot is more difficult to explain. Part of the loss of information comes from the fact that the signal to noise is worse for the phonon delays. The speed of sound is $\sim 2 \mathrm{~cm} / \mu \mathrm{s}(\sim 1 \mathrm{~cm} / \mu \mathrm{s}$ in $\mathrm{Si})$ giving a timescale for our delays of $\sim 1 \mu \mathrm{s}$. As shown in Sec. 2.1.4, the inductance of the input coil begins to reduce signals on this timescale. Additionally, the delay is constructed from the time the pulses in each channel reach $20 \%$ of their peak value. Since the peak value of a phonon trace is a good estimate of the energy in the channel, this definition of delay is correlated with the phonon energy. As a result, the delay plot is weakly correlated with the box plot which folds back for the reasons discussed earlier. 


\section{Chapter 4}

\section{The Phonon Lookup Table}

An important aspect of the CDMS analysis is the phonon "Lookup Table Correction." The "Lookup Table Correction" is a method of homogenizing the detector response throughout the entire crystal to achieve improved signal to noise. In particular, the phonon timing parameters vary substantially across the surface of the detector. The "Lookup Table Correction" is critical to the implementation of the cut in phonon timing.

\subsection{The Algorithm}

\subsubsection{Event Selection}

The lookup tables are generated from bulk electron recoils within the inner electrode. Typically large gamma calibrations provide the events from which the table is constructed. A cut, known as the Qinner cut, demanding that the ionization in the outer electrode be consistent with noise, guarantees that the events for the table are not close to the outer edge of the detector. A cut on the $\chi^{2}$ of the ionization pulse and prepulse baselines serves to remove pileup events. There are a few remaining problematic events that survive the Qinner and pileup cuts. These events are tagged by having an event location that is not on the 2-dimmensional manifold as in Fig. 3.5. Such events are removed by hand and are believed to be internal multiple scatters. 
It is important to make every effort to insure that the events used for the construction of the table correspond to bulk electron recoils. In particular, we must also make sure that surface electron recoils are not included since they would contaminate the correction of phonon timing parameters.

\subsubsection{Linearizing the Energy Response}

The first step in constructing the lookup table is to remove any energy dependence of the parameters that are going to be corrected. Each phonon timing parameter, $\tau_{j}^{i}$ has a three parameter energy correction of the form

$$
\widetilde{\tau_{j}^{i}}=\frac{\tau_{j}^{i}}{a+c \pi^{b}}
$$

where $\pi$ is the estimate for the total phonon energy and $a, b$, and $c$ are determined empirically. $i$ is an index over the three timing parameters (pdel, pminrt, and pminrt4070), and $j$ is an index over the four phonon channels. In Eqn. (4.1), we are free to choose an overall normalization for $\widetilde{\tau}_{j}^{i}$. Typically, all the channels of a particular timing parameter are normalized to the same value.

The phonon energy estimate is also corrected by

$$
\tilde{\pi}=\lambda\left(e^{\pi / \lambda}-1\right)
$$

where $\lambda$ is empirically determined.

\subsubsection{Averaging over nearest neighbors}

It is useful to first consider the following simple correction algorithim. We can implement a crude position correction by taking our delay parameters and defining a set of bins as shown in Fig. 4.1. A correction value for each bin is calculated from events inside of the bin. Events are then corrected based upon which bin they reside in. This kind of correction was the first attempt to account for position dependence when the effect was first discovered in 1999. Such a correction is effective as long as 

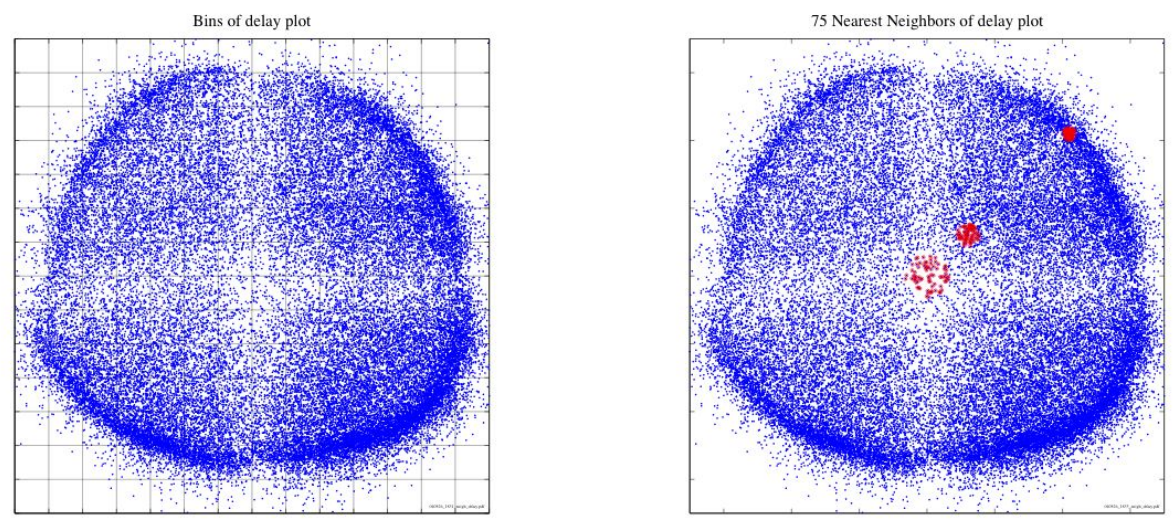

Figure 4.1: left: A generic delay plot with a uniform binning scheme shown by the grid. right: a 75 nearest neighbor binning scheme where 3 clusters of 75 nearest neighbors are shown. Note the variation of the "size" of each cluster for different positions in the delay plot.

the variation within each bin is small. There are two complications to the simple correction algorithim described above. As discussed in Sec. 3.1.2, the delay parameters alone are degenerate in mapping the event location. Thus, instead of using just the delay parameters, we can use the 3D parameter described at the end of Sec. 3.1.2 and divide the space into bins in the three coordinates. The second problem is that the event distribution is non-linear in all of our parameters. Thus, in the bins closer to the rim of the detector, the variation is actually quite high. This complication can be addressed by allowing the bin sizes to vary in the space. However, determining the correct bin sizing is extremely difficult. The non-linearity exists in all 3 position parameters and varies throughout the entire space as well as from detector to detector.

Blas Cabrera came up with the current correction algorithim which addresses the non-linearity of the position parameters. The current correction scheme basically 
corresponds to a redefinition of what a "bin" is. To illustrate the scheme, it is easiest to look again at the two dimensional delay plot shown in Fig. 4.1. Instead of defining a bin as corresponding to ranges in the two delay parameters, we define a bin as a cluster of $N$ neighboring events, as given by the plot. We can immediately see that as long as the physical density of the events is uniform throughout the detector, this "binning" scheme yields a constant physical volume per bin. The variation in the "size" of the cluster in the delay parameters is automatically accounted for as shown in Fig. 4.1.

In practice, the $3 \mathrm{D}$ position parameter is used instead of the delay parameters alone. For every event given by Sec. 4.1.1, a "bin" is constructed corresponding to the event's $N$ nearest neighbors. As with the simple correction, for each bin of $N$ events, the correction value is calculated as the average of the $N$ events in the bin. The outlined binning scheme definitely "overbins" the parameter space, however, it guarantees that we have full coverage. The result is a map of correction values to the coordinates of each event satisfying our selection criteria which is referred to as the "lookup table."

The choice of $N$ cannot be arbitrary. Qualitatively, one can determine the dependence of the correction on $N$ by considering the two extreme cases: $N \sim$ total number of events in the table and $N=1$. If $N$ is the total number of events in the table, averaging will yield the same value for all coordinates. Thus, the lookup table would only correspond to an overall scaling and the $x-y$ position dependence would not be accounted for. For $N=1$, the situation is reverse. In this case, every point in the table is it's own correction, and the net result is to make all values the same which would avearge away not only the $\mathrm{x}-\mathrm{y}$ position dependence, but any position dependence on depth as well.

\subsubsection{Applying the Correction}

The lookup table corrections can be applied to any target data series including the data from which the table was constructed. As described in Sec. 4.1.3, the lookup table assigns a set of correction values to a particular coordinate. For each event in 
the target data set, the correction value would be that of the lookup table coordinate closest to the event's coordinate as given by the 3D parameter. Two additional correction related quantities are also calculated. The first is the distance between the event coordinate and the lookup table coordinate. The second is an estimate of the "size" of the lookup table bin. This parameter is the average of the distances between the lookup table coordinate and each of the $N$ points in its "bin." The belief is that these two parameters can be combined to assess whether a correction is valid. It turns out that things are actually a little complex since the differences in the phonon spectra for nuclear recoils causes the majority of events to be at the extrema of the box plot.

\subsection{The Lookup Table in Real Life}

The first application of the Lookup table correction was during SUF Run 21 [37]. Detector testing revealed that Z1 had a sufficiently large $T_{c}$ gradient so that the position dependence of the phonon amplitude would wash out the discrimination based on ionization yield. Effects due to the gradient had been initially removed with a correction similar to the simple method described in Sec. 4.1.3. However, once it was discovered that the delay parameters were also double valued, a new method needed to be determined. Blas Cabrera first developed the lookup table algorithim as a way to salvage the usefulness of Z1's ionization yield parameter. Only two parameters were corrected: the total phonon energy and the risetime of the summed phonon trace. It turns out that the correction also improved the response of the other detectors. Of particular importance is the fact that the correction made it possible for all of the detectors to have a meaningful rejection of surface events based on phonon timing. It is worth noting that since Run 21 was the first application of the Lookup table correction, a number of things were still being developed. In particular, the Lookup tables averaged over $O(20)$ neighbors for each detector and the risetime parameter was not corrected for energy dependence.

The next implementation of the correction was for the analysis of data during the beta calibration of G31 at Berkeley. During these runs, the detector was exposed to an 
external photon source $\left({ }^{60} \mathrm{Co}\right)$, an external neutron source $\left({ }^{252} \mathrm{Cf}\right)$, and a collimated internal beta source $\left({ }^{109} \mathrm{Cd}\right)$. The primary focus of the studies was to characterize the detector response to betas on either side of the detector at different charge biases.

Figs. 3.6 shows that the ZIPs have both clear separation of surface events in risetime and significant variation of the risetime across the detector surface. The purpose for the lookup table is clear. Though the risetime discrimination is good at any given spot on the detector surface, the variation in the risetime signal over the entire detector makes it difficult to define a risetime cut that can be applied globally. As described in Sec. 3.2.2, the position dependence is due to effects that are specific to each detector and its bias settings. As a result, defining a position dependent risetime cut is also difficult since the position parameterization must be unique for each detector configuration. The correction uses the detector as a self calibration to account for the variation in detector response making it possible to utilize the phonon timing information to reject surface events.

During the G31 studies, we learned that the lookup table is extremely sensitive to contamination from surface electron recoils. Obviously a correction generated from surface electron recoils will average out the expected faster phonon risetime. It turns out that low energy gammas also interact sufficiently close to the surface to have faster phonon risetimes (see Fig. 3.6). These events should also be excluded from the set of events used in constructing the table. It also became apparent that the phonon timing parameters needed to be corrected for energy dependence. Additionally, the studies indicated that the risetime and starttime of the phonon signal in the dominant channel are more effective discriminators for surface events.

\subsection{The Lookup Table and Soudan R118}

For Soudan R118, the following quantities are position corrected :

- pt, prg, pr: phonon energy estimates where pt is the total phonon energy (intrinsic plus Luke), prg is the recoil energy estimate assuming the event is an electron recoil, and pr is the recoil estimate where the ionization is used to estimate the Luke phonon contribution 
- ptrt: the $10-40 \%$ risetime of the summed phonon pulse

- pdel: the delay of the primary channel (the channel with the most energy) relative to the ionization pulse (QIst)

- pminrt: the 10-40\% risetime of the primary channel

- pminrt4070: the 40-70\% risetime of the primary channel

- pfrac: the fraction of total energy in the channel directly across from the primary channel

The correction for pminrt4070 is wrong because of an error in the code.

The Lookup table has bin sizes that are $N \sim O(75)$ events. The principle governing the choice of $N$ was that $N$ should be sufficiently large so that the volume occupied by the bin should span the entire resolution of the radius parameter.

Two of the lookup tables have problems. The first problem is for Z4. In constructing the table, a few events with especially noisy ionization pulses passed the event selection criteria. The noise does not affect the risetime or the phonon energy estimates, but it had a substantial impact on the delay. Specifically, these events caused a few entries in the lookup table to have negative correction values. The number of entries is small, and miscorrected events are removed by a cut demanding that the delays be positive. The second problem is with Z1. The cuts used to remove outliers that are off the $2 \mathrm{D}$ position manifold were not sufficiently stringent and may cause the lookup table to be less effective for certain coordinates.

Figs. 4.2 and 4.3 show the effects of the lookup table on the yield and total risetime for Z1. It is clear that there is substantial improvement. It is also clear that things can be better. Part of the inefficiency of the position correction is that the physical distribution of events is not uniform throughout the detectors. As a result, for some regions of the detector, the choice of $N$ may be either too small or too large.

It may be possible to further improve the post correction phonon pulse shape discrimination by improving the lookup table event selection criteria. For the current lookup tables, all events in the electron recoil band down to $5 \mathrm{keV}$ were accepted. Studies in G31 show that low energy photons can have both full charge collection and 


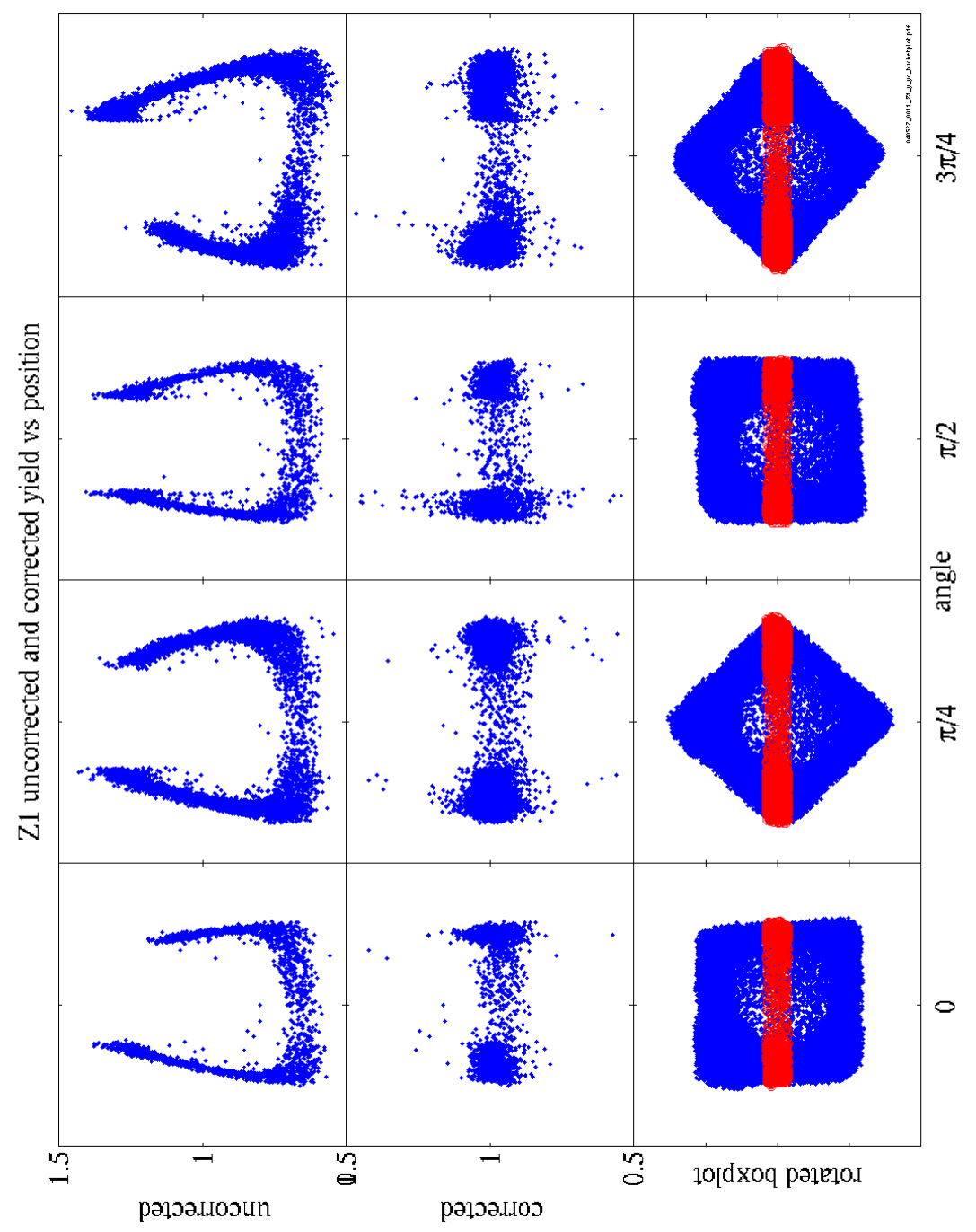

Figure 4.2: Z1 yield vs position before (top row) and after (middle row) the correction

faster phonon signals because they interact sufficiently close to the detector surface for the enhanced down conversion of the intrinsic phonons while being sufficiently deep so that charge collection is complete. Including these events washes out the 


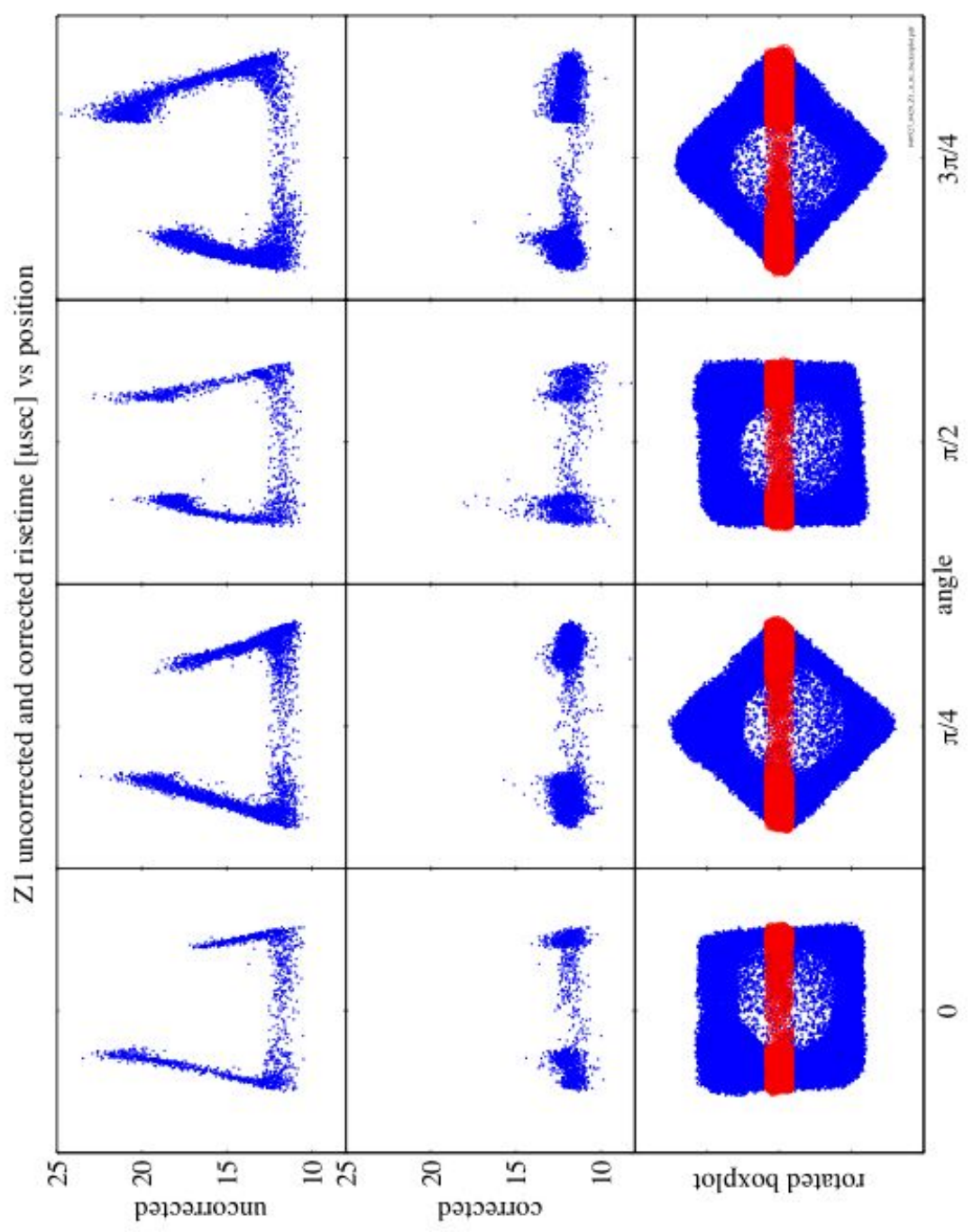

Figure 4.3: Z1 total risetime vs position before (top row) and after (middle row) the correction

risetime discrimination. Demanding that events for the lookup table be sufficiently high in energy may improve things. 


\section{Chapter 5}

\section{The CDMS II Soudan Installation}

\subsection{The Soudan Mine}

The CDMS II experiment resides on the 27th level of the Soudan Underground Mine. The mine is part of the Minnesota state park system and is approximately 100 miles north of Duluth, MN. Access to the experimental area of the mine is possible only via a "cage ride" down a single shaft.

Fig. 5.1 shows a diagram of the CDMS II area in the underground laboratory. The shielding, detectors, cryostat, and front end electronics are in a RF shielded clean room. The pumps, cryogens, and gas bottles are on the cryo pad. All the computers and electronics for data acquisition and detector control (except for the front end electronics) are in a room on the mezzanine.

In addition to the area underground, CDMS II also has a room in a building at the surface. This room houses the computers for the analysis farm as well as computers for controlling the cryogenic and data acquisition system. The extensive use of computers to remotely control the majority of the experiment is extremely helpful since the mine does not have guaranteed access during all hours of the day. 


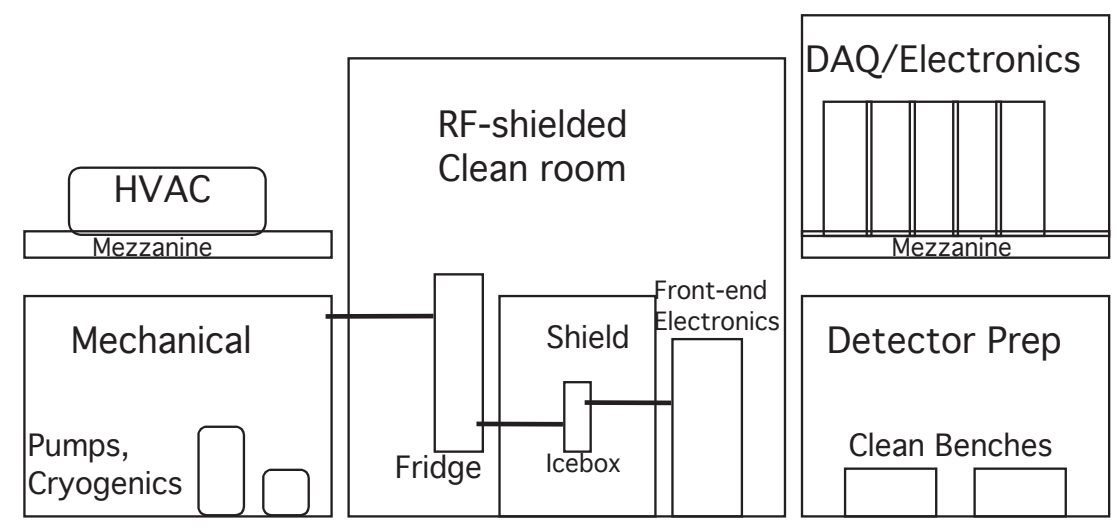

Figure 5.1: Diagram of the CDMS II lab area in the Soudan Underground Laboratory.

\subsection{Cryogenics}

Six cocentric copper cans form the "Ice Box." The innermost can holds the detectors and has a volume of $\sim 21 \mathrm{~L}$. Copper stems connect each can of the Ice Box to one of the thermal layers of an Oxford Instruments S-400 dilution refrigerator (left side of Fig. 5.2). Fig. 5.2 shows a diagram of the connection of the Ice Box to the fridge. The advantage of this arrangement is that the external room temperature shielding described in Sec. 5.3 shields the detectors from any radioactivity in the cryostat.

A large number of cryogenic operations can be performed via a computer. The integration of a computer into the control system has proven extremely helpful in the mine where access is restricted. In particular, it is possible to conduct most routine cryogenic procedures from a building at the surface.

The system is expected to bring the detectors to a base temperature of $20 \mathrm{mK}$. Currently, the detectors are at a temperature that is a little less than $50 \mathrm{mK}$. There are a number of possible causes for the elevated base temperature. It is possible that the heat sinking of the striplines is not effective. Since the striplines connect the cold electronics to the warm electronics through the e-stem (right hand side of Fig. 5.2), they can provide a good thermal link between the innermost layers and room 


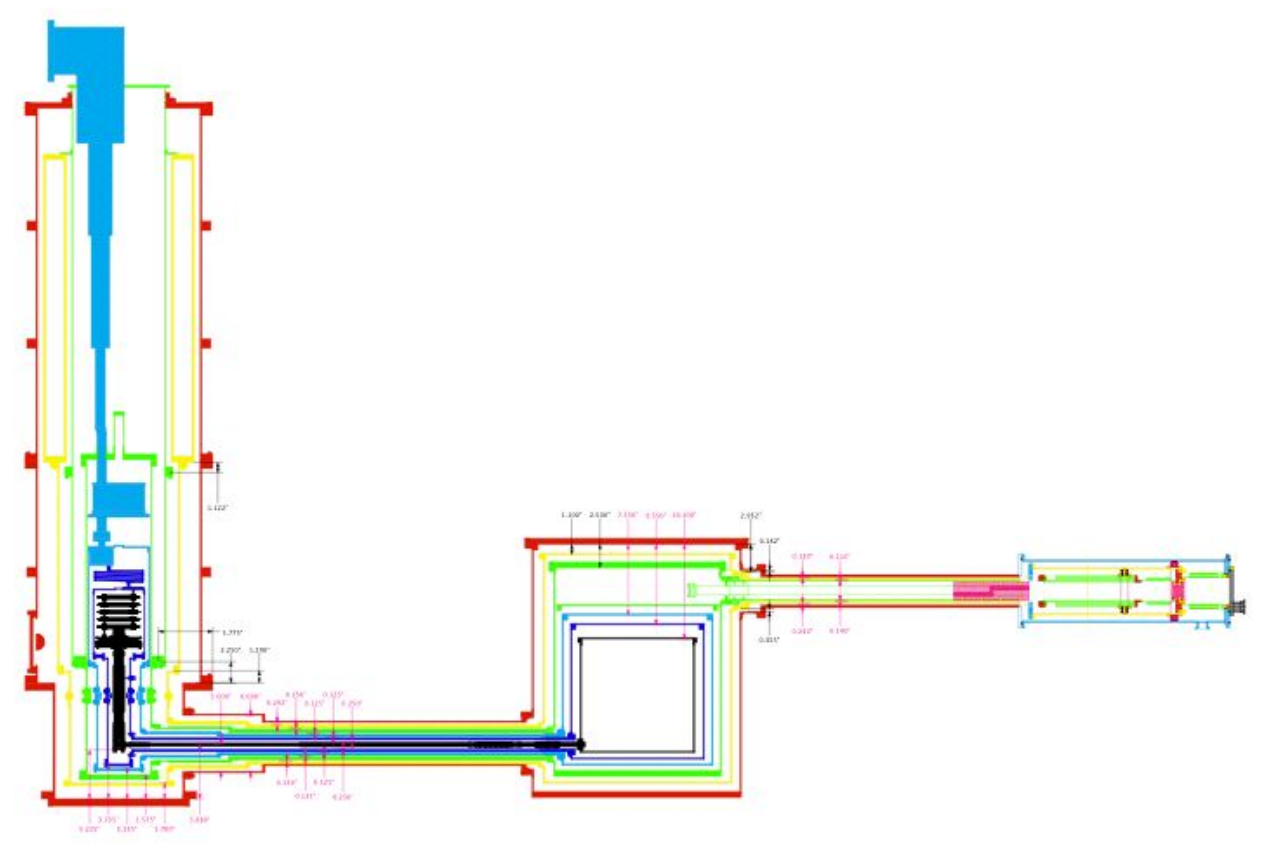

Figure 5.2: A diagram outlining the layout of the connection of the Ice Box (center) to the Oxford cryostat (left). The temperatures of each layer (outermost to innermost) is supposed to be $300 \mathrm{~K}, 77 \mathrm{~K}, 4 \mathrm{~K}, 600 \mathrm{mK}, 50 \mathrm{mK}$, and $20 \mathrm{mK}$.

temperature. Additionally, the absence of radiation baffles for the $600 \mathrm{mK}$ can leaves a direct line of sight from the inner most can to the $4 \mathrm{~K}$ layer. It would be fortuitous if these two heatloads are the only cause of the elevated base temperature since they are relatively easy to address. However, there are other possibilities that are not so easily handled. In commisioning the system, we found that the Ice Box and stems were badly misaligned. We realigned the setup in order to ensure that the system would cool down without having the thermal layers touch while still maintaining good thermal contact with the fridge. It is possible that in realigning the system, we work-hardened the copper reducing its thermal conductivity. Additionally, the wiring along the c-stem (the stem connecting the Ice Box to the fridge) may be thermally connecting one layer to another. Finally, there is considerable noise in the 
fridge instrumentation. This noise may deposit a significant amount of power into the system.

During one of the initial runs of the Soudan cryostat, there was an unfortunate accident where the LHe bath had been pumped out while the surrounding vacuums were still at atmospheric pressure. The consequence was a crumpling of the bath wall similar to how one might crumple a soda can. Blas Cabrera worked very hard to smooth out the bath in an attempt to restore it to its intended configuration.

All recent fridge runs suffer from a leak from the LHe bath to the OVC (outer vacuum chamber). This leak can lead to a catastrophic failure of the system if we do not constantly pump on the OVC. The hypothesis is that He from the leak condenses on the cold interior surfaces of the OVC eventually saturating the surfaces. Once the cold surfaces are saturated, the He remains as a gas and shorts the cold layers of the OVC to room temperature thereby rapidly releasing a significant quantity of the condensed He gas. We call this process a "burp" since it results in a rapid spike in the pressure in the OVC. If a burp is sufficiently large, the system can be lost. It is unclear what the cause of the leak is. It may be due to a failure in the Oxford refrigerator, the damage to the bath, or an error in assembling the system.

For the current run, a "LN band aid" reduces the leak. The band aid consists of freezing a small amount of LN at the base of the LHe bath which plugs up some of the leak. We also continually pump on the OVC.

Despite all the efforts to reduce the accumulation of He, there are still intermittent burps. A burp usually boils away a significant amount of the LHe in the bath and expels a small amount of mixture resulting in a warming of the system. If the $600 \mathrm{mK}$ layer warms up past $1 \mathrm{~K}$, it is impossible to cool it back down without turning off the detector electronics. A possible explanation for this is that when the system warms up, the "flyovers" connecting the SQUID cards to the FET cards become normal. The normal flyovers are good thermal conductors and short the $600 \mathrm{mK}$ layer to the 4K stage. Turning off the detector electronics restores the flyovers to their intended superconducting state. Recycling the power for the detector electronics results in some small instability in the experimental configuration since it is never guaranteed that the electronics return to exactly the same state. After a burp, we usually have 
to zap all the SQUIDs and use the LEDs to reneutralize the detectors before we can resume routine data taking.

\section{$5.3 \quad$ Shielding}

The CDMS II experiment has an overburden of $780 \mathrm{~m}$ of rock, or 2090 mwe (meters water equivalent). This overburden reduces the surface muon flux by a factor of $5 \times 10^{4}$. The neutron background due to muons scattering in the rock is expected to be $\sim 300 \times$ lower at Soudan than at SUF.

In addition to the overburden, an average of $22.5 \mathrm{~cm}$ of lead and $50 \mathrm{~cm}$ of polyethelene surrounding the icebox shield the detectors from photons and neutrons respectively. The additional shielding around the icebox makes contamination from all radioactivity external to the icebox negligible.

40 paddles of $5 \mathrm{~cm}$ thick plastic scintillator enclose the CDMS II shielding. The enclosure serves as a muon veto system. Highly ionizing particles generate a large signal in the veto system making it possible to effectively tag muons that pass through the veto enclosure. We deem events to be related to cosmogenic activity in the shielding if there is significant veto activity within a $50 \mu$ s pre-trigger window. Fiber

optics along the face of each paddle of the veto system connects the scintillator to a blue LED pulser. The pulser is used for routine monitoring of the veto performance. Fig. 5.3 shows a diagram of the CDMS II active and passive shielding assembly. 


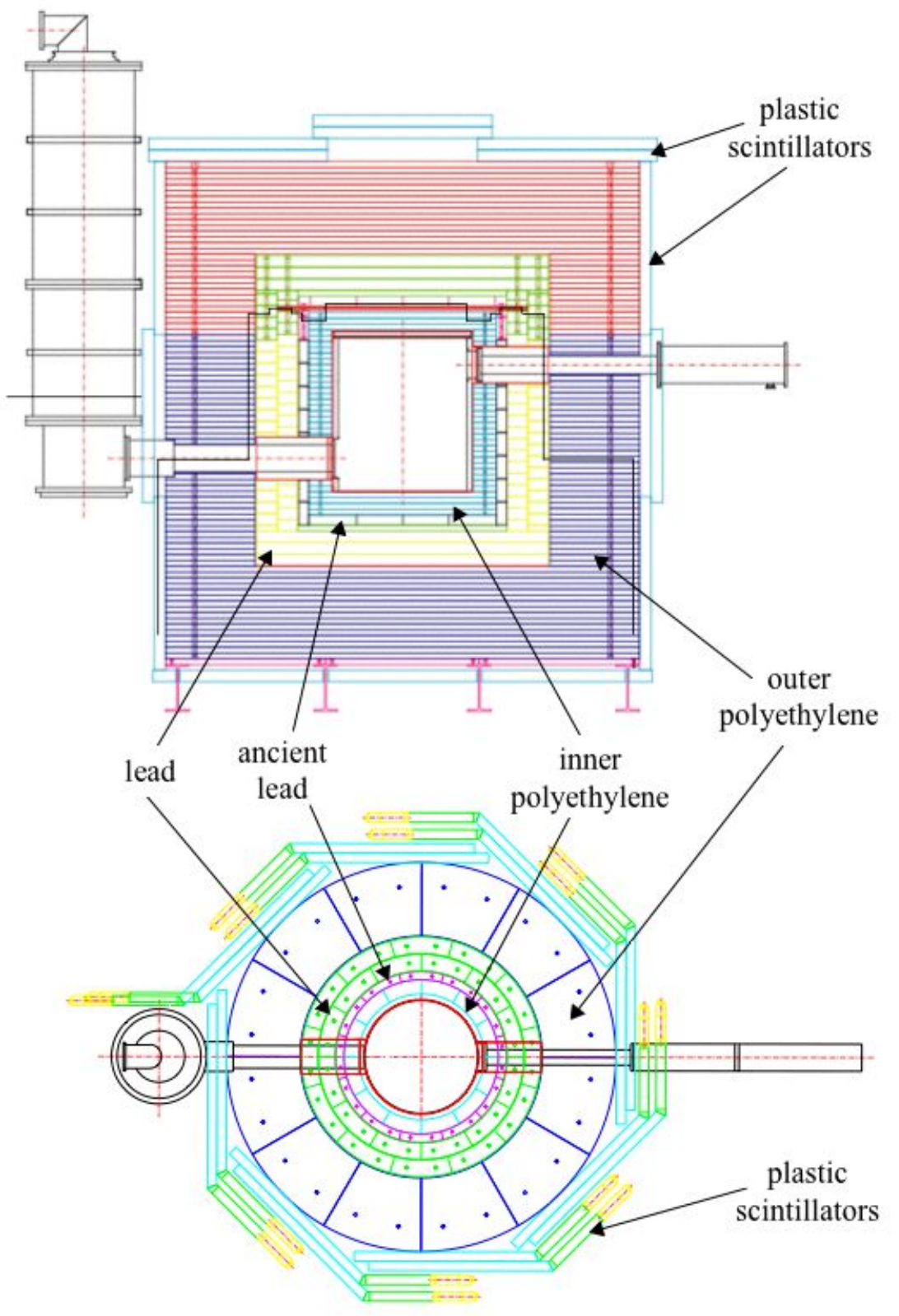

Figure 5.3: Diagram of veto and shielding for the CDMS II installation from the side and top 


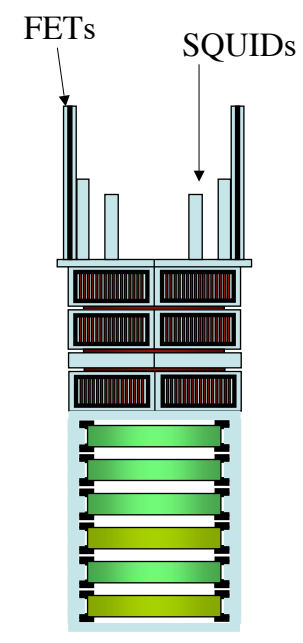

Figure 5.4: CDMS II Tower 1. Z1 is at the top of the Tower and Z6 is at the bottom

\subsection{Electronics and Data Acquisition}

Fig. 5.4 shows the packaging of the CDMS II ZIP detectors. Each "Tower" holds six ZIP detectors together with their cold electronics. The structure of the Tower not only provides the mechanical support for the detectors, but also the heat sinking to the various thermal layers of the icebox. At the top of each Tower there are six pairs of cards which contain the cold electronics for each detector. Each pair of cards consists of a FET card and a SQUID card connected via a superconducting "flyover." The FET cards are weakly heat sunk to the $4 \mathrm{~K}$ thermal layer and are heated to a temperature of $\sim 130 \mathrm{~K}$ for optimum noise performance. The SQUID cards are heat sunk to the $600 \mathrm{mK}$ layer, also for noise performance. The "flyover" connects the two cards electronically while isolating them thermally. 
A crate just beyond the e-stem (right most side of Fig. 5.2) houses the Front End electronics. The Front End electronics are custom made $9 \mathrm{U}$ boards and contain all the room temperature electronics required for controlling and reading out the cold hardware. The Front End electronics also amplify the detector output signal before sending it to another set of custom $9 \mathrm{U}$ boards in the electronics room. The boards in the electronics room (RTF boards) condition and filter the signals and generate digital trigger signals. 14-bit Struck digitizers (SIS 3300) digitize the waveforms of the detector signals.

The signals from the veto PMTs are sent directly to a set of custom $3 \mathrm{U}$ boards in the electronics room where they are integrated and stretched for easy digitization. NIM discriminators and logic generate digital trigger information for the veto system. 12-bit Joerger digitizers (VTR812) digitize the wave forms of the integrated veto pulses.

The digital trigger information for both the veto and detector system is recorded in a digital history buffer (Struck SIS 3400). A custom 9U Trigger Logic Board processes all of the digital trigger information and determines whether the signal satisfies the conditions for a global trigger. If the TLB issues a global trigger, the digital history buffer and all of the digitizers are read out by the Data Acquisition System. All custom electronics are controlled via GPIB.

The online data acquisition software is custom made. Three features are important. The first feature is the interface through JAVA. This interface makes it possible to control the experiment from secure locations that are physically far from the hardware. Most importantly, the experiment can be controlled either from the CDMS II control room in the surface building or from computers in one of the residences. The second important feature of the DAQ is its ability to take data at $\sim 40 \mathrm{~Hz}$. This increased throughput (compared to the system at SUF) reduces the time spent on acquiring calibration data while simultaneously providing larger calibration data sets. Lastly, the data acquisition is automated for routine WIMP search data acquisition. The automation minimizes the opportunity for user errors in data taking.

The DAQ generates data files each of which contain no more than 500 events. Once a file is complete, the DAQ copies the file to a computer analysis cluster at 
the surface through a high speed fiber optic connection. Files are grouped together into "Series" which correspond to a single period of uninterrupted data acquisition. At the beginning of each series, the DAQ reconfigures all of the hardware and takes 500 random triggers prior to taking data. In normal low background running, the DAQ stops data acquisition just prior to cyrogenic transfers, terminating the "Series." During the cryotransfer, the DAQ automatically performs two routine activities: it flashes the detector LEDs to remove any space charge build up, and it acquires veto pulser data for veto diagnostics and monitoring. Thirty minutes after the completion of the cryotransfer, the DAQ automatically resumes data acquisition by initiating a new "Series."

\subsection{Online Data Analysis}

A cluster of seven linux machines in the surface building carry out the online rapid data analysis of the files produced by the DAQ. Once analysis is complete the raw data and the output of the analysis are archived to tape. The DAQ also maintains a second separate archive of the raw data files.

The online analysis is broken up into two parts. The first part, referred to as "Dark Pipe," performs the basic pulse shape analysis of the digitized wave forms as well as initial analysis of the digital history. Dark Pipe references all time stamps in the history buffer to the global trigger of the event and calculates a number of additional trigger timing quantities. The pulse shape analysis of the veto pulses consists of a peak finding algorithim which determines the amplitude and time of the maximum point of the veto trace within a specified window of the global trigger.

Dark pipe analyzes the detector pulses using two algorithims: one in the frequency domain and one in the time domain. The time based algorithims are better estimates of the pulse heights for high energies. For the ionization pulses, the time based algorithim is a four parameter $\chi^{2}$ fit. For the phonon pulses, the algorithim is straight forward integration. For optimum resolution at low energy, we use a frequency domain optimal filtering alogrithim to estimate the peak height (area) of the ionization (phonon) pulse. We construct a noise spectrum from the initial 500 random events 
in each series. This noise spectrum is combined with a template pulse to generate the filter for the signal ${ }^{1}$. Convolving the optimal filter with the wave form yields an estimate for both the pulse amplitude and the pulse start time. Since the ionization pulse shape varies very little, we can generate the pulse template for the ionization channel from an average of real ionization pulses. The phonon pulse template is just a simple double exponential with an appropriate risetime and falltime. Since the phonon pulse shape varies, the use of a single filter constructed from one template leads to slight misestimates of the energy in the phonon system which we correct offline using the "Lookup Table Correction."

Dark pipe also uses a time based algorithim to determine the time of various points along the pulse. All Dark pipe output is generated on a file by file basis where eight files are generated for each event file from the DAQ.

"PipeCleaner" is the second part of the online analysis. Unlike Dark Pipe, it does not run on an event file by event file basis. It runs on a series by series basis. Prior to analysis by PipeCleaner, all of the Dark Pipe output files are merged into a single set of eight files for the entire series. For each series, PipeCleaner applies the required calibrations and corrections to generate the final set of reduced quantities of interest.

\footnotetext{
${ }^{1}$ Details of optimal filtering can be found in the appendix of [43].
} 


\section{Chapter 6}

\section{The Deep Site Measurement: Soudan Run 118}

\subsection{Run Overview}

From October 11, 2003 until January 11, 2004, the CDMS II experiment conducted a search for WIMPs. The data taken during this time period is referred to as Run 118. Throughout the course of data taking, routine calibrations were performed by inserting one of three external radioactive sources through a small tube in the shielding beside the e-stem. The sources are

- a $2 \mu \mathrm{Ci}{ }^{133} \mathrm{Ba}$ source: This source illuminates the detectors with photons providing data for characterizing the detector response to electron recoils. The source has prominent lines at 303,356 , and $384 \mathrm{keV}$ which we use for calibrating the ionization measurement.

- a $1 \mathrm{mCi}{ }^{133} \mathrm{Ba}$ source: This larger Ba source is used for its high rate allowing the acquisition of a large number of electron recoil events in a short time. The source is usually placed a few $\mathrm{cm}$ into the shielding so that the majority of the photons reaching the detectors are due to Compton scattering in the cans. This configuration makes the illumination of the detectors somewhat more uniform. Unfortunately, when the source is in this position, the lead shielding obscures 
the lines. Total insertion of the source results in an event rate that is too high. During the first half of December, a set of large calibrations was taken using this source. These calibrations provide the events needed for calculating the look up table described in Ch. 4 and for determining the phonon timing cuts.

- a $1 \mathrm{mCi}{ }^{252} \mathrm{Cf}$ source: This source illuminates the detectors with both photons and neutrons providing the experiment with an in-situ neutron calibration. Since the neutrons can activate materials inside the shielding and the detectors themselves, use of this source is minimized. Only three neutron calibrations were performed: once at the end of November, once mid-December, and once early January.

Apart from the calibrations, there are only three situations where WIMP search data was not obtained

- Routine cryogenic maintenance: The cryogenic system requires filling twice a day. Each fill takes one and a half hours before data taking can be resumed. During the cryogenic filling, we also perform other routine maintenance operations such as monitoring the veto via a blue light pulser and neutralizing the detectors using LEDs.

- Unexpected cryogenic problems: The fridge "burps" about once a month (see Sec. 5.2). Each "burp" expels some mixture and warms the detectors to $\sim 1$ $\mathrm{K}$ making data acquisition impossible. It usually takes a few hours to restore routine operations after a burp.

- Operator error and poor noise: Throughout the course of the run, there are occasional errors by the operators of the experiment. The frequency of these errors is significantly reduced since the majority of the data acquisition is fully automated by the DAQ. There are also periods of substantially increased noise. Evaluation of the noise spectra allows for easy identification of these periods which are subsequently removed from the data used for the WIMP search.

For Run 118, the experiment triggered on the summed phonon pulses for each detector. Thresholds were set so that $\sim 30 \%$ of the triggers are noise. We estimate 

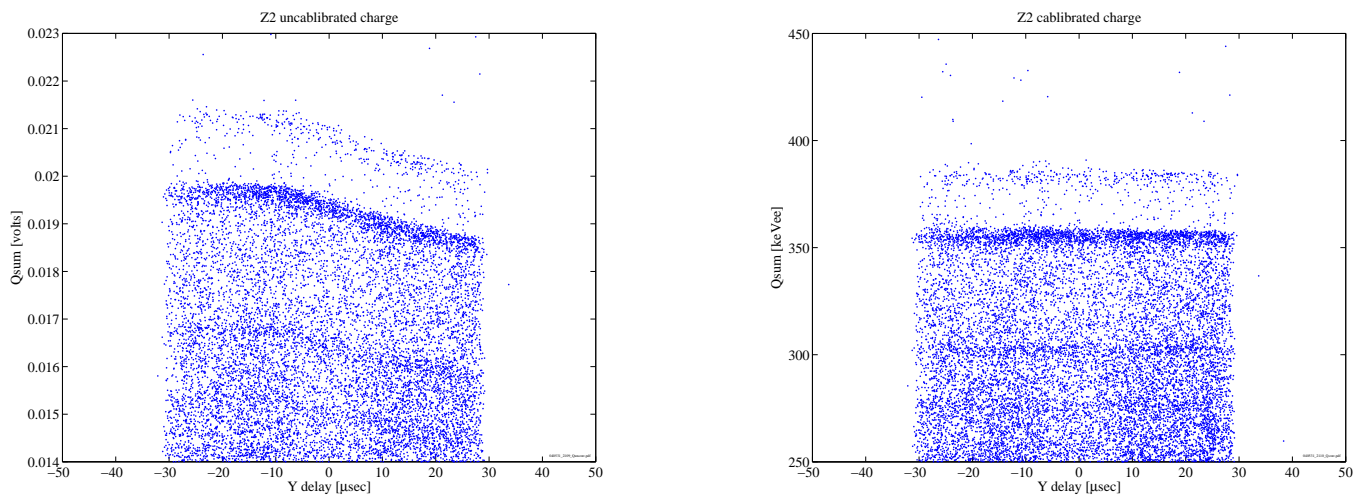

Figure 6.1: Position dependence (left) of the ionization response due to currently unknown causes. This position dependence can be removed (right) and is negligible at lower energies.

the trigger efficiency for each detector by considering events throughout the entire WIMP search data which triggered on another detector. The trigger efficiency is the fraction of those events for which the appropriate trigger bit is set within $100 \mu$ s after the global trigger. Fig. 6.9 shows a plot of the trigger efficiency estimate.

Additional details of Run 118 are found in [63].

\subsection{Energy Calibration}

\subsubsection{Ionization}

\section{Position Dependence}

The ionization for the detectors have a small position dependence shown in Fig. 6.1. The cause of this position dependence is yet to be determined. One possibility is that minute damaging of the crystal during detector fabrication and processing may yield a position dependence to the distribution of charge trapping defects. If this is the case, the position dependence of the ionization signal should also be present in the signal due to Luke phonons. In Run 118, the position dependence was removed during the charge calibration. 


\section{Lines}

The ionization channel is calibrated to the $356 \mathrm{keV}$ peak from the ${ }^{133} \mathrm{Ba}$ calibration as shown in Fig. 6.2. The excellent alignment of the two other distinct peaks (at $303 \mathrm{keV}$ and $384 \mathrm{keV}$ ) as well as agreement with Monte Carlo simulations indicate that the ionization is linear and accurate to better than a few percent. Additional confirmation of the calibration comes from the $10.4 \mathrm{keV}$ in the WIMP search data after neutron calibrations as shown in Fig. 6.3. This peak is the Gallium line coming from activation of the Ge by neutrons. The Si detectors do not see any lines with out current calibration statistics and are calibrated to agree with the Monte Carlo spectrum. With increased statistics, the Si ZIPs can be calibrated to shared lines with the Ge detectors.

\subsubsection{Phonons}

The phonon channels are calibrated to the ionization for gamma calibrations. Again, the $10 \mathrm{keV}$ Gallium line (Fig. 6.4) serves to confirm the calibration at low energies. Additionally, the recoil spectrum for neutrons agrees with Monte Carlo simulations which not only confirms the validity of the calibration, but also the claim that the phonon measurement measures the absolute recoil energy. 


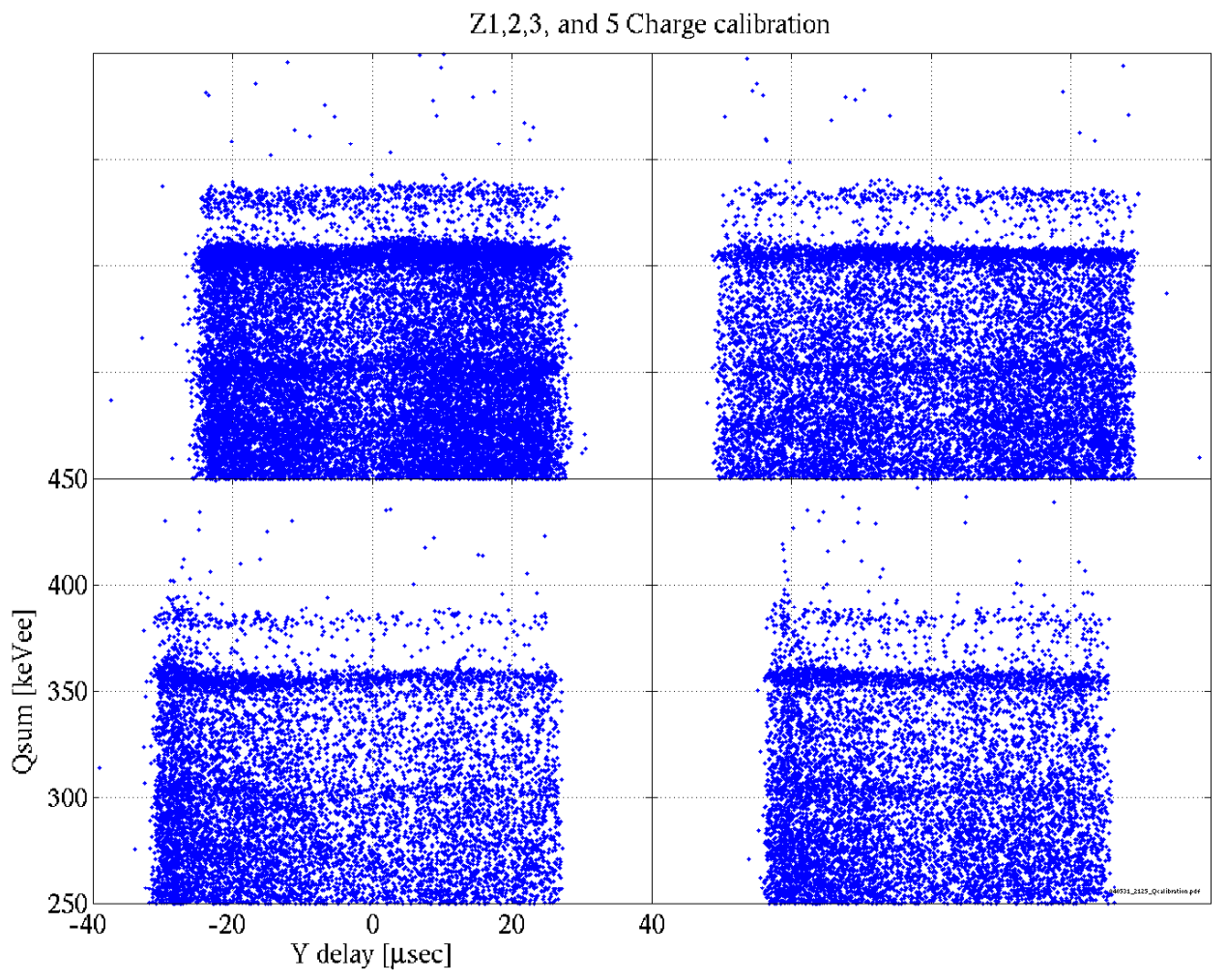

Figure 6.2: Charge calibration of the 4 Ge ZIPs. Z1 (top left), Z2 (top right), Z3 (bottom left), and Z5 (bottom right). 


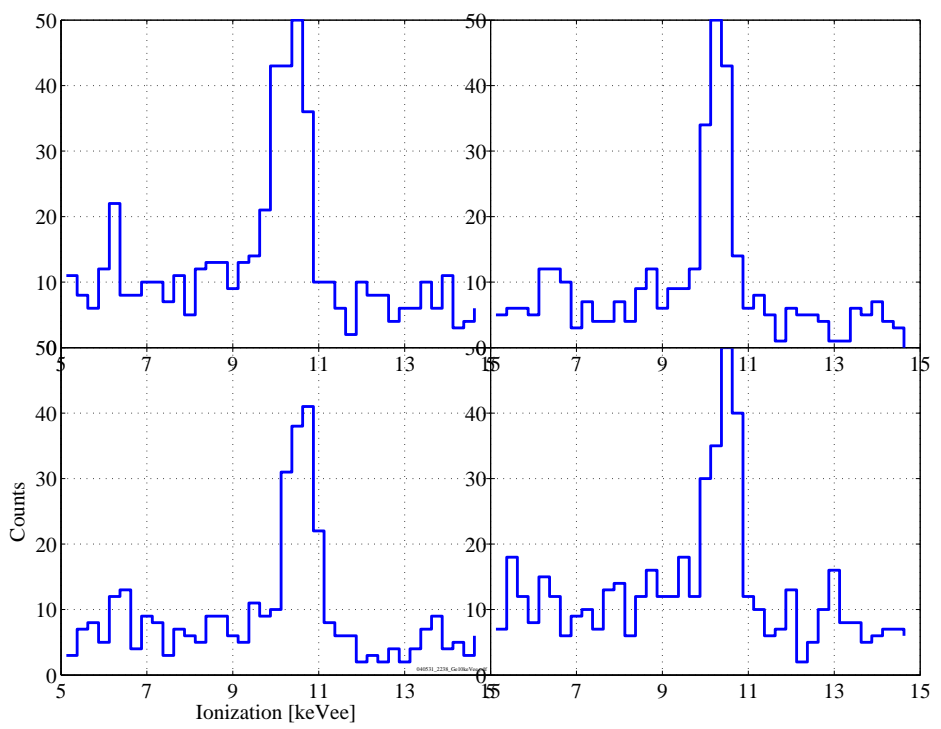

Figure 6.3: 10 keVee line in ionization from activation of Ge by the neutron calibrations.

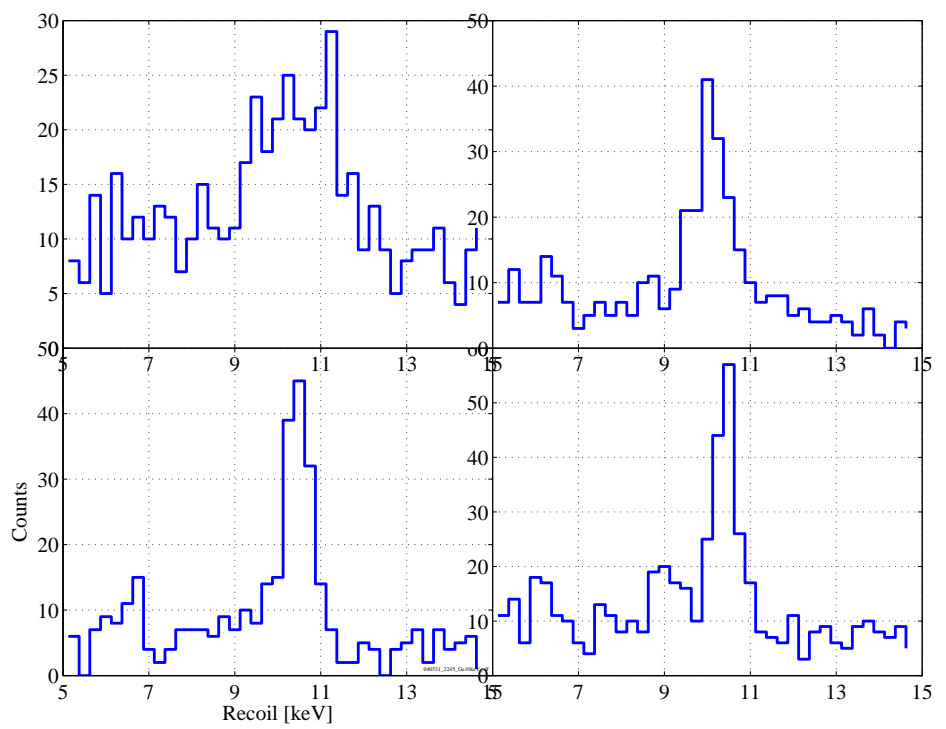

Figure 6.4: $10 \mathrm{keV}$ line in phonons from activation of Ge by the neutron calibrations. The line for Z1 in phonons is wider since the correction is worse at low energies. 

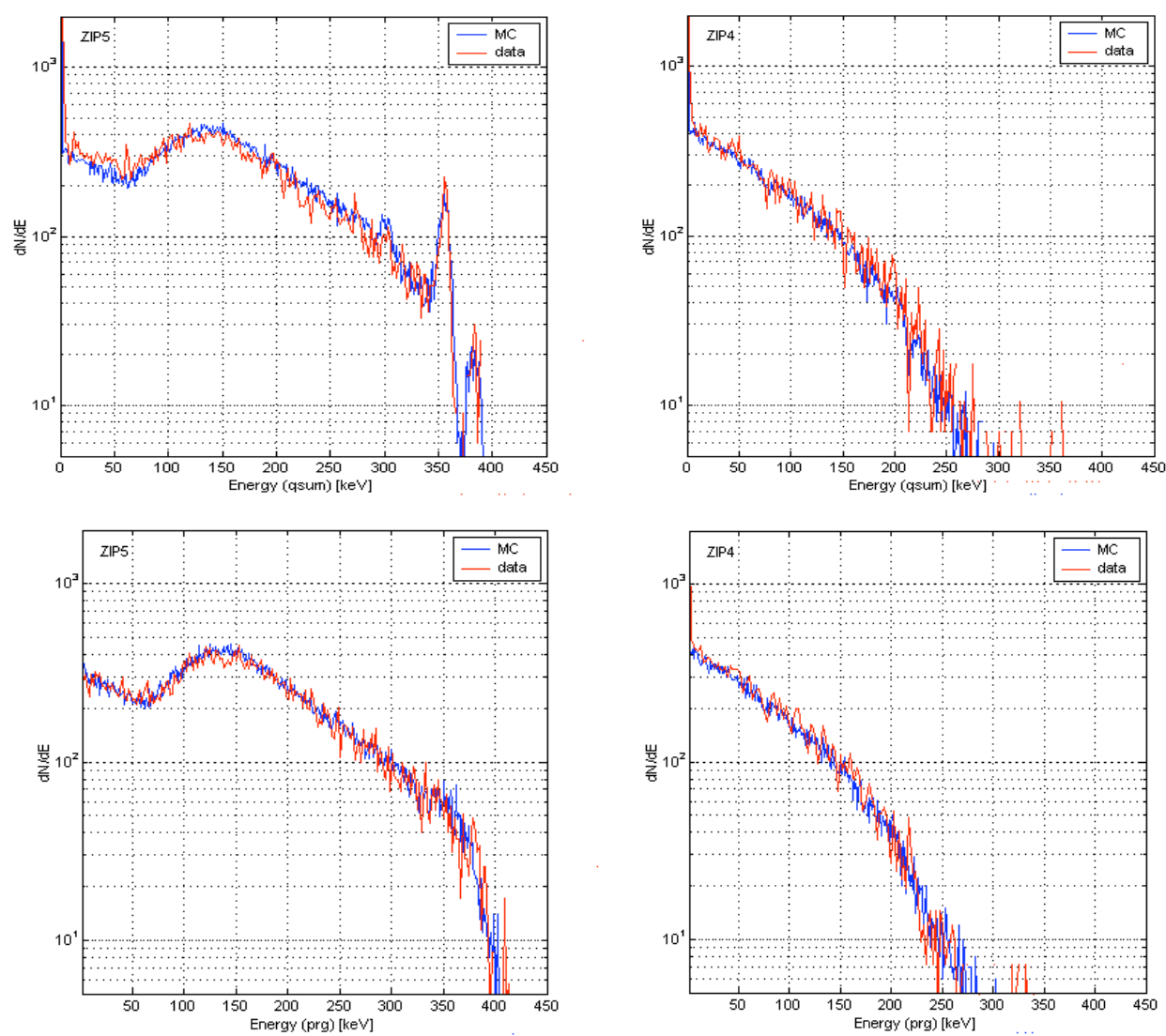

Figure 6.5: Comparison between Monte Carlo and data for Ge (left) and Si (right) detectors. Top plots are ionization spectra for gammas from ${ }^{133} \mathrm{Ba}$. Bottom plots are recoil spectra for neutrons from ${ }^{252} \mathrm{Cf}$. The agreement confirms the phonon and ionization calibrations as well as the claim that the phonon energy measurement is unquenched. 


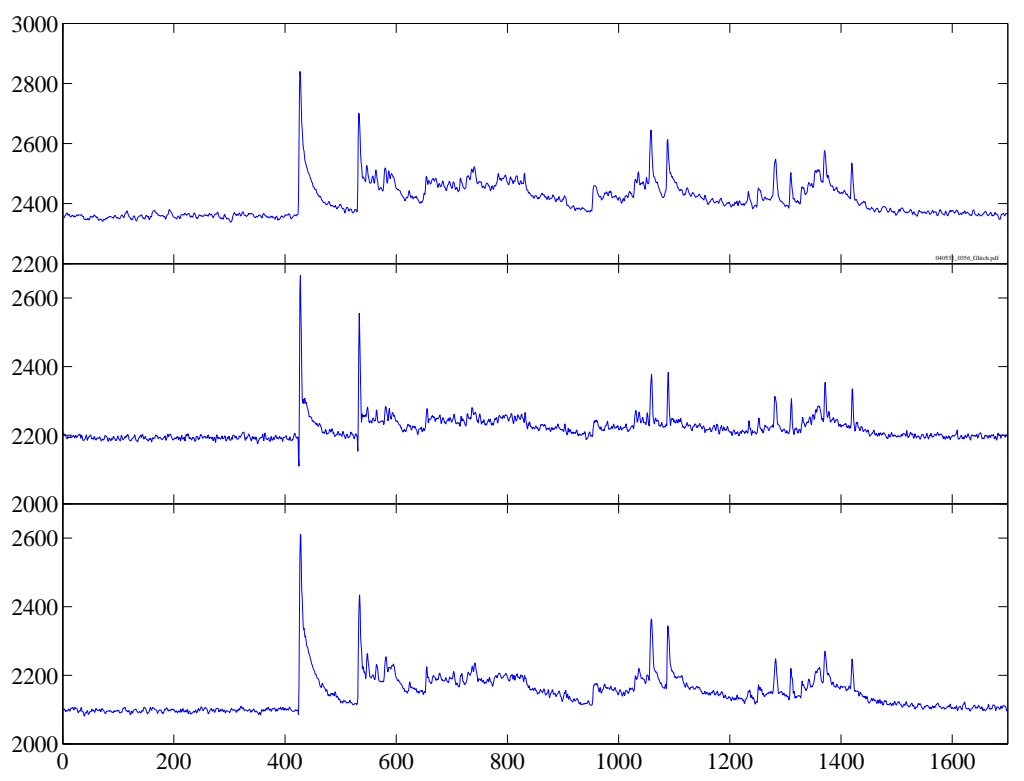

Figure 6.6: Traces for phonon channel A in Z1, Z2, and Z3 for an electronics "glitch." Such events are rejected by demanding energy in only one detector.

\subsection{Cut Definitions}

In addition to the Nuclear Recoil cut and Phonon Timing cut defined in Secs. 2.3.2 and 2.3.3, we apply the following cuts:

- Muon Anti-coincident cut: We reject events with activity in the muon veto within a $50 \mu$ s pre-trigger window. This cut guarantees that all events passing this cut cannot be caused by muon scattering within our shielding.

- Single scatter cut: Since WIMPs will not multiply scatter, we reject events for which the phonon energy is larger than noise $(>6 \sigma)$ in more than one detector. This cut also rejects triggers on large noise glitches that are associated with the Soudan system (see Fig. 6.6).

- Analysis Thresholds: To insure that our analysis is insensitive to small changes in our noise, we impose analysis thresholds. We demand that the recoil be larger 

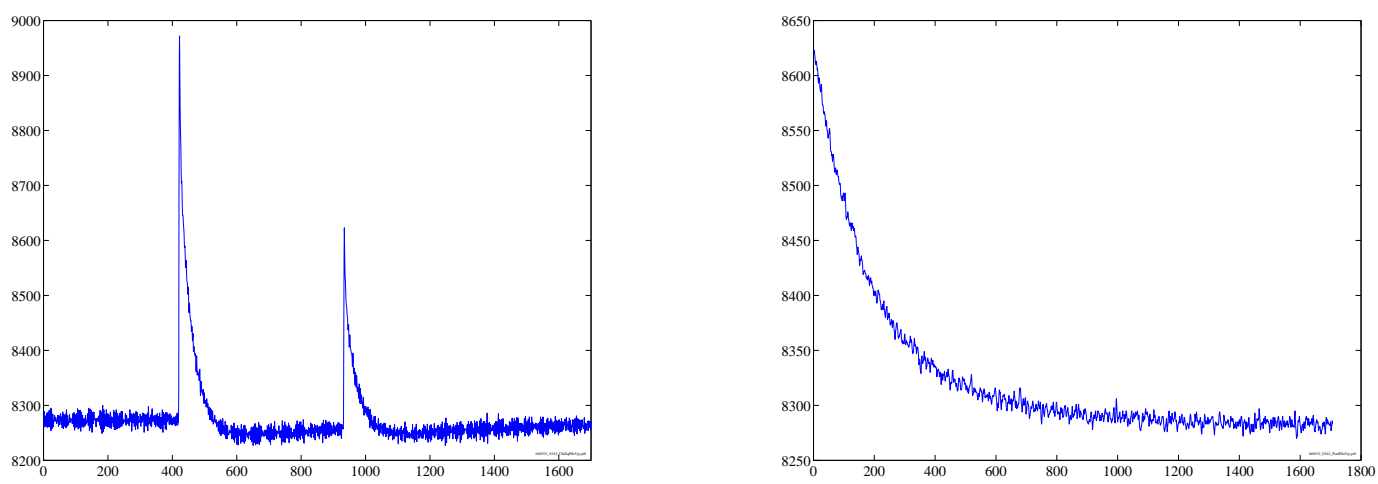

Figure 6.7: Traces illustrating the two classes of pile up events. The first class (left) have events occuring after the trigger and are rejected based on the $\chi^{2}$ of the ionization pulse. The second class (right) have events occuring before the trigger and are rejected based on the standard deviation of the pre-pulse baseline for the phonon channels.

than $5 \mathrm{keV}$ (20 keV for Z1). and that the ionization in the inner electrode be larger than 1.5 keVee. Additionally, since the expected WIMP recoil will be at low energies, we only look at events with recoil $<100 \mathrm{keV}$.

- Pile Up: Pile up events are problematic for our pulse shape analysis. There are two classes of pile up events: events that occur after the triggered event and events that occur prior to the triggered event. Examples of these events are displayed in Fig. 6.7. The first class of events are rejected based on the $\chi^{2}$ of the ionization pulse. The second class of events are rejected based on the standard deviation of the phonon pre-trigger baseline.

- Qinner: Events interacting near the outer rim of the detector can have reduced charge collection due to charge trapping on the surface and inhomgenieties in the electric field near the surface. As a result, we reject all events for which the measured ionization in the outer electrode (also referred to as the guard ring) is inconsistent with noise. 


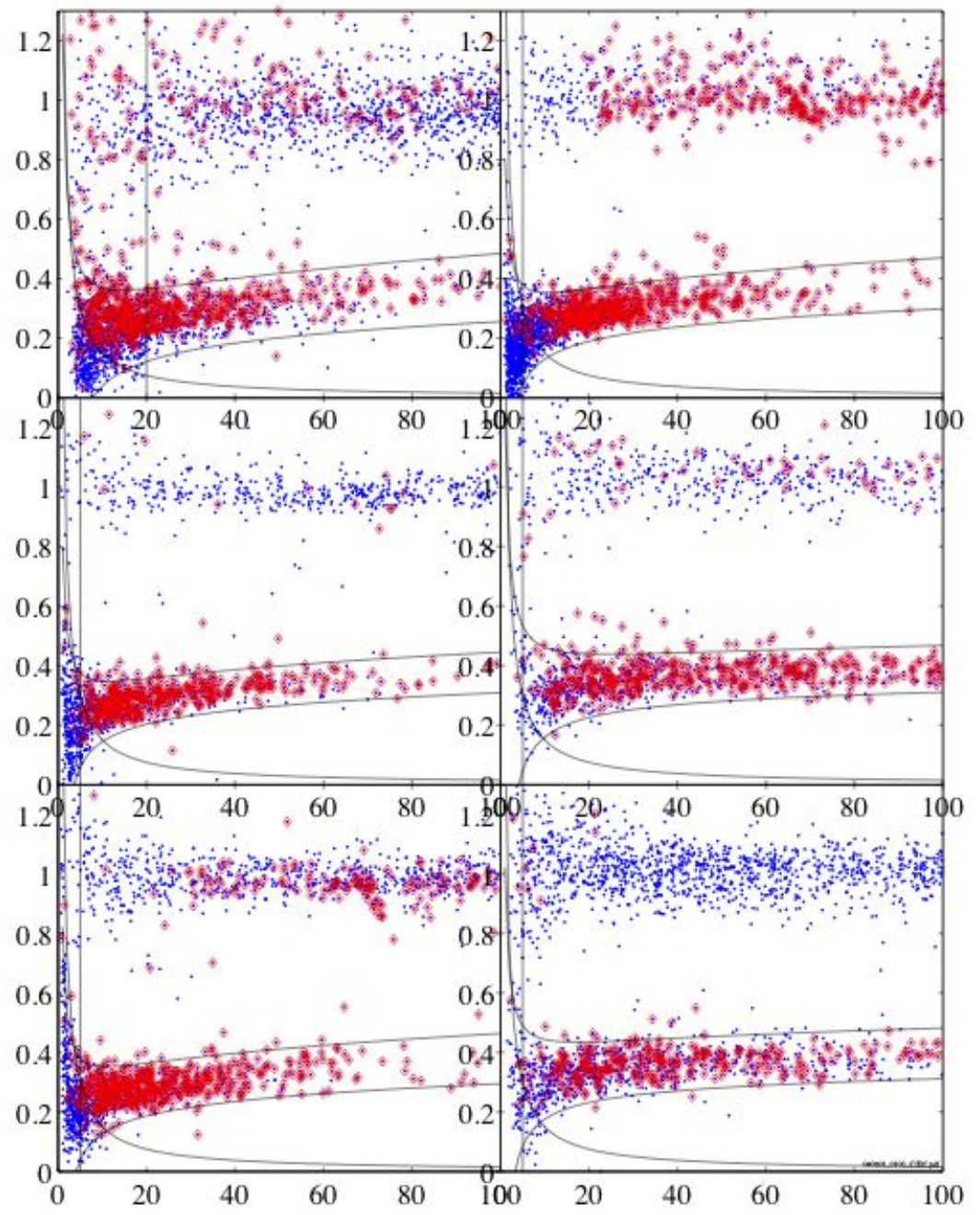

Figure 6.8: Effects of threshold cuts, timing cuts, and nuclear recoil band on neutrons for Z1 (top left), Z2 (top right), Z3 (middle left), Z4 (middle right), Z5 (bottom left), and Z6 (bottom right). Blue dots correspond to single scatter inner electrode events after pile up rejection. Red diamonds are events passing phonon timing cuts. Solid lines indicate the analysis thresholds and the nuclear recoil acceptance band. Compare with Fig. 7.1. 


\subsection{Efficiencies}

In order to calculate our exposure to WIMPs, we need to estimate the loss in efficiency of each of our cuts on WIMPs. Figs. 6.9 and 6.10 show plots of the efficiencies for each detector.

\section{Veto}

The digitization window for each event is $1.7 \mathrm{~ms}$. The veto coincidence window is 50 $\mu$ secs giving a $3 \%$ loss in livetime. The veto is estimated to have a $>99 \%$ efficiency in tagging muons that pass through the enclosure.

\section{Analysis Thresholds}

Fig. 6.8 shows the effects of the analysis thresholds on a neutron calibration. The efficiency of the ionization threshold is determined from the neutron calibrations. We estimate the efficiency to be the fraction of events within a $2.5 \sigma$ nuclear recoil band that pass the ionization threshold cut.

\section{Qinner}

We also use the neutron calibration to estimate the efficiency of the Qinner cut. The efficiency is defined as the fraction of events in the Nuclear Recoil band and passing the risetime cut that also pass the Qinner cut. Using calibration data to estimate the efficiency of the guard ring cut is conservative since WIMPs will interact uniformly throughout the detector whereas neutrons, though more penetrating than photons at low energies, are still not entirely uniform. Additionally, neutrons are more likely to scatter several times within a single detector whereas WIMPs will only scatter once. These discrepancies between neutrons and WIMPs have the most impact on our efficiency estimate at higher energies where the neutron statistics are low. At low energies, this estimate of the efficiency of the guard ring cut is consistent with efficiencies calculated by Monte Carlo simulations. 


\section{Pile Up Cuts - $\chi^{2}$ and phonon standard deviation}

Since the two pile up cuts are sensitive to the noise in the data, the efficiencies for the two pile up cuts are calculated directly from the photons in the WIMP search data in order to account for variations in the detector noise over time. The efficiency of each cut is simply the fraction of events in the Electron Recoil band passing the cut. Since the $\chi^{2}$ is applied to the ionization pulses, the energy dependence for WIMPs will be that of the photons scaled by the centroid of the nuclear recoil band in order to account for the quenching.

\section{Phonon Timing Cuts}

The efficiency of the phonon timing cuts is calculated from the neutron calibrations as the fraction of events in the nuclear recoil band passing the timing cuts. From Fig. 6.8 we see that the increased Luke phonon content for electron recoils results in a different efficiency when the timing cuts are applied to electron recoils.

\section{Ionization Yield Cut}

The efficiency of the ionization yield is also calculated from the neutron calibrations. It is the fraction of events within $3 \sigma$ of the nuclear recoil centroid and passing the phonon timing cuts that are also in the nuclear recoil band. Fig. 6.8 illustrates the efffects of the threshold cuts, timing cuts, and ionization yield cut on neutrons for each detector.

\subsection{Background Estimates}

There are three backgrounds for the experiment: neutons, photons, and betas from radioactive contamination. Table 6.1 summarizes our background estimates for the WIMP search data. 


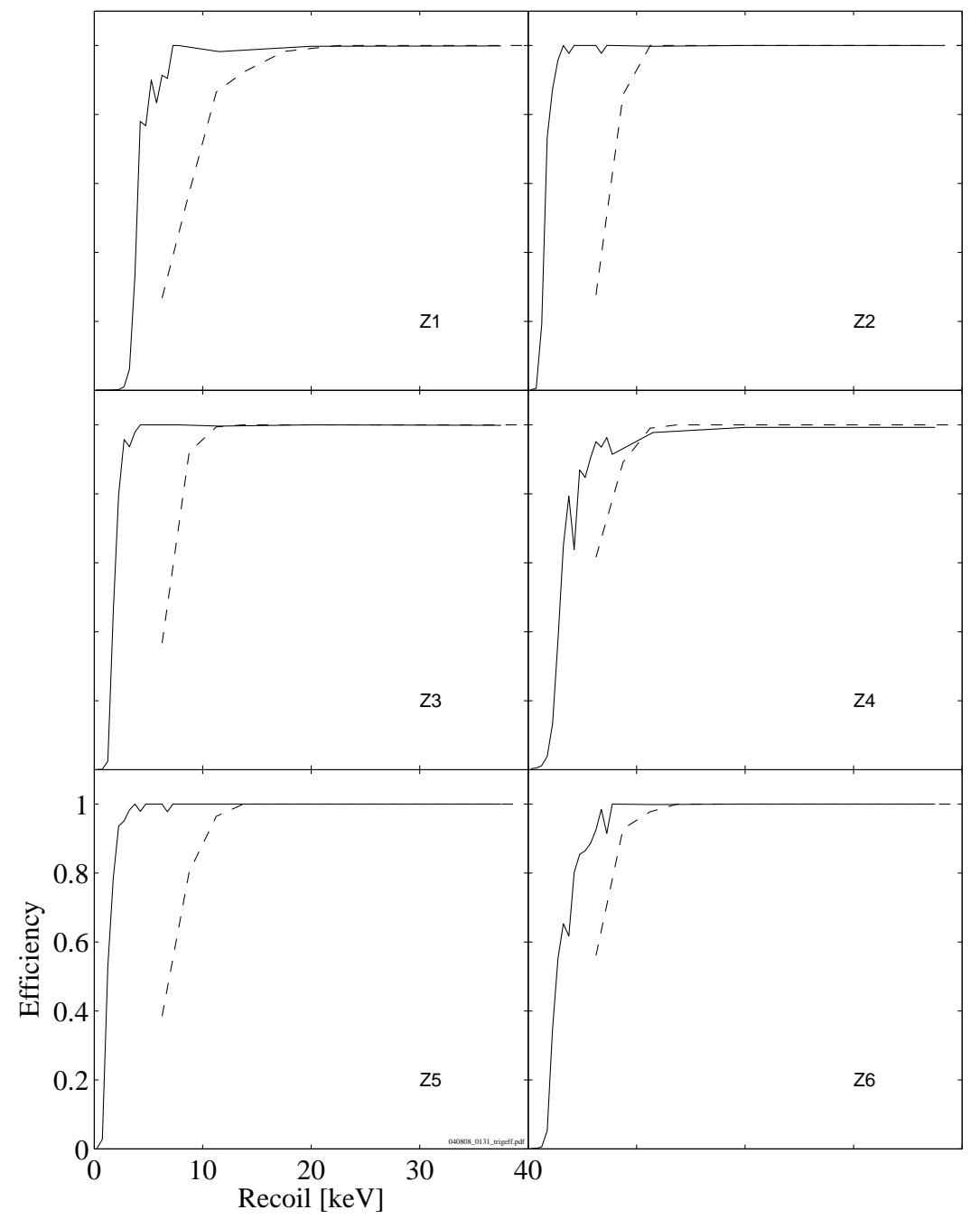

Figure 6.9: Hardware trigger (solid) and ionization analysis threshold (dashed) efficiencies versus recoil. The hardware trigger efficiency is unity above our phonon analysis thresholds.

\subsubsection{Neutrons}

Monte Carlo simulations of neutrons [65] produced by cosmogenic activity in the rock and the shield predict that throughout the entire WIMP search data, we should see 
$1.9 \pm 0.4$ neutrons coincident with activity in the veto and $0.05 \pm 0.02$ anti-coincident neutrons. We find no neutrons coincident with veto activity which is consistent with the Monte Carlo predictions. We estimate the muon veto to tag muons with an efficiency that is $>99 \%$. Our estimated background from anti-coincident neutrons is 0.05 events.

\subsubsection{Photons}

Photons create two backgrounds for the experiment. The first background consists of bulk electron recoils that pass all of our cuts. The second background consists of "ejectrons." Since we expect the misidentification rate in both of these cases to be proportional to the total number of photons that are correctly identified as electron recoils, we combine these two backgrounds into a single background estimate. For determining the expected background due to external photons, we take care to only use data from gamma calibrations that were not used in determining the phonon timing cuts. We find a total of 36,005 single scatter events between our analysis thresholds and in the $2 \sigma$ electron recoil band. We find 77 events in the nuclear recoil band of which only one passes the phonon timing cuts. We calculate the photon misidentification fraction to be $1 / 36,005=0.0028 \%$ with a $90 \%$ upper limit of $3.9 / 36,005=0.01 \%$. In the WIMP search data, there are 6,700 single scatter events in the electron recoil band in the same energy range, giving an expected photon background of 0.2 events with a $90 \%$ upper limit of 0.7 events.

\subsubsection{Radioactive Betas}

Simulations of "ejectrons" and contamination betas suggest that "ejectron" multiple scatters which have poor charge collection in both detectors share the same depth profile as betas from radioactive contamination on the detector surface. For this estimate, we define a "beta band" corresponding to the region in between the electron recoil band and the nuclear recoil band. As in the case with the photon background estimate, we again take care to only use gamma calibrations that were not used to determine the phonon timing cuts. For each detector we define a "beta-beta double" 
as a multiple scatter with an adjacent detector in which the event is fully contained in the inner electrode for both detectors and has an ionization yield that is less than $2 \sigma$ of the electron recoil band in both detectors. Of the beta-beta doubles, 401 events are in the beta band of both detectors of which 28 are in the nuclear recoil band of the given detector. No events in the nuclear recoil band pass the phonon timing cuts. These numbers give an estimated misidentification fraction of single scatter betas of 0 and an upper limit of $2.3 / 401=0.57 \%$. The WIMP search data has 163 single scatter events in the beta band giving a $90 \%$ upper limit on the estimated contamination beta background of 0.94 events. This calculation is overly conservative since we know that some fraction of the single scatter betas in the WIMP search data is actually caused by the external photons and is already accounted for in the photon background estimate.

We can estimate the number of single scatter beta band events due to external photons by using the calibration data. For the 36,005 single scatters, there are 577 single scatters in the beta bands. We calculate the expected number of photon induced beta band single scatters in the WIMP search data to be $6,700 \times 577 / 36,005=107.37$ events. This leaves an estimate of 55.63 single scatter beta band events in the WIMP search data and a $90 \%$ upper limit of 0.32 events for the beta leakage from radioactive contamination.

An alternative method of estimating the beta leakage from surface contamination is found in [63]. This method uses only the calibration data used to set the timing cut and takes advantage of the fact that the cut is determined in such a way as to reject all of the events in the calibration data. It can be shown that if a cut is set so that it always rejects all $N$ events in a calibration data set, then on average we can estimate the leakage to be $\frac{1}{N+1}$. Using this method, we estimate the beta background due to contamination to be $0.4 \pm 0.2$. This estimate is inconsistent with the beta-beta estimate described earlier indicating that there may still be significant differences between the depth profile for "beta-beta doubles" and that of contamination betas. We take the more conservative estimate of 0.4 events. 


\begin{tabular}{lr} 
Type of Leakage & Expected Leakage for entire WIMP search \\
\hline Gamma Leakage & 0.2 \\
Betas from contamination & 0.4 \\
Neutrons & 0.05
\end{tabular}

Table 6.1: Table listing expected leakages for gammas, contamination betas, and neutrons.

\subsection{Stability}

\subsubsection{Stability of the Ionizaton and Recoil Calibrations}

To confirm the stability of our ionization calibrations, we look at the ionization spectra for three $2 \mu \mathrm{Ci}{ }^{133} \mathrm{Ba}$ calibrations taken at different times. We use calibrations taken from 12/05-12/16, 12/16-12/18, and 1/6. Fig. 6.11 shows that the peaks in Ge consistently line up. For each of the Ge ZIPs, the Kolmogorov-Smirnov test yields a $>50 \%$ probability that the spectrum for either of the first two calibrations is drawn from the parent distribution of the third calibration indicating that the ionization calibration is stable.

We evaluate the stability of the phonon calibration by studying the electron recoil bands in the WIMP search data. If the phonon calibration is stable, the means of the bands should not deviate significantly from unity. We divide the WIMP search data into four subsets of data: 10/11-11/1, 11/1-11/20, 11/20-12/15, and 12/15-1/11. For each subset, we calculate the mean and width of events in the Electron Recoil bands in a few energy bins. Fig. 6.12 shows the means and widths of the four subsets and Fig. 6.13 shows the deviation of the means from unity. The phonon calibration for the Ge ZIPs varies by $<5 \%$. 


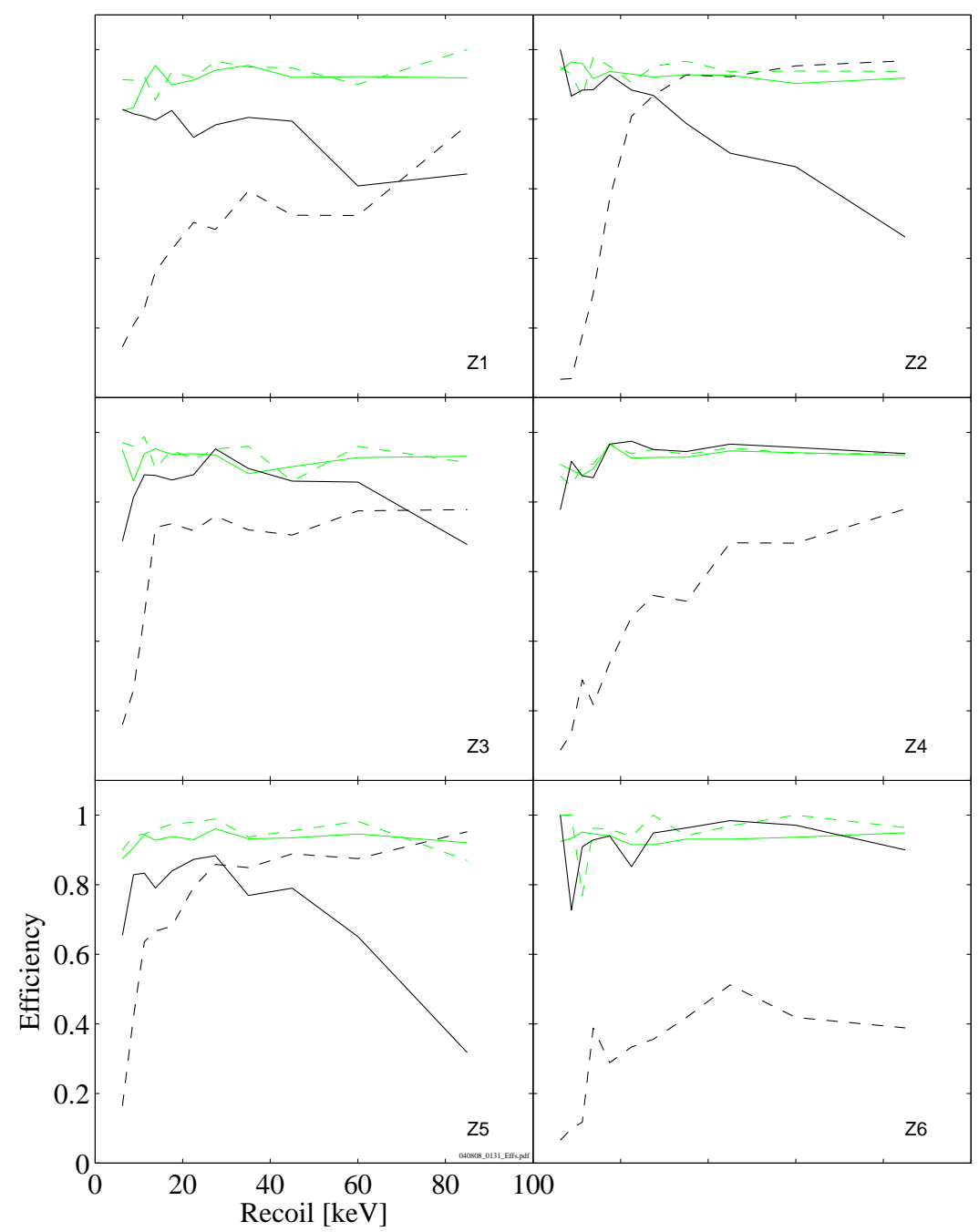

Figure 6.10: Efficiencies versus recoil for the Pile Up Cut (solid green), Nuclear Recoil Cut (dashed green), Qinner Cut (solid black), and Phonon Timing Cut (dashed black). Note that the Phonon Timing cut reduces the overall efficiency at lower energies while the Qinner cut dominates the efficiency at high energy. The Qinner efficiency drops at high energies due to low statistics and non-uniformity in the illumination of the detector. The efficiency for WIMPs is expected to remain constant making this estimate conservative. The impact of this drop should be marginal since there is very little expected WIMP signal above $50 \mathrm{keV}$. 


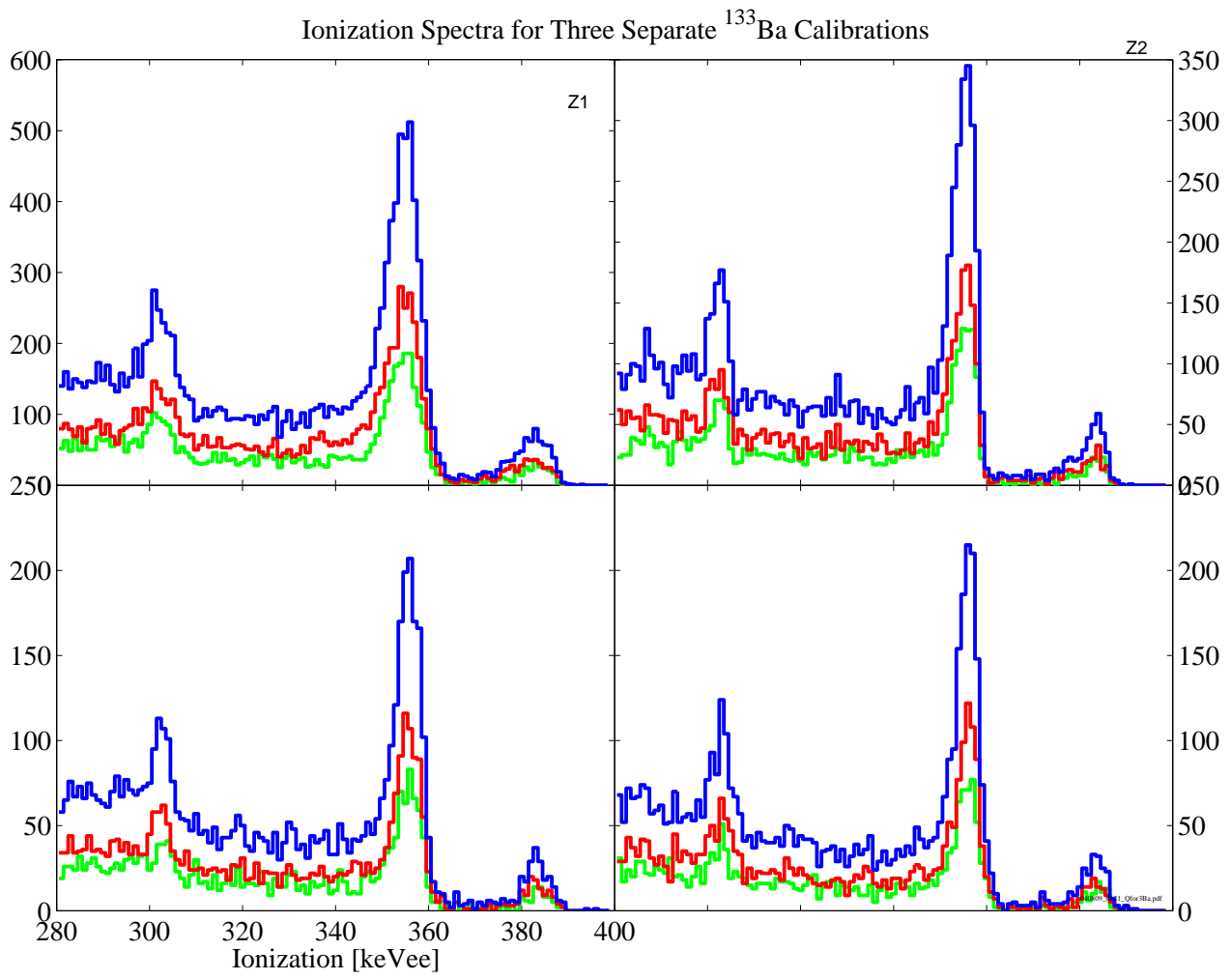

Figure 6.11: Ionization spectra of three $2 \mu \mathrm{Ci}{ }^{133} \mathrm{Ba}$ calibrations: $12 / 05-12 / 16$ (green), 12/16-12/18 (red), and 1/6 (blue) for the Ge ZIPs. 


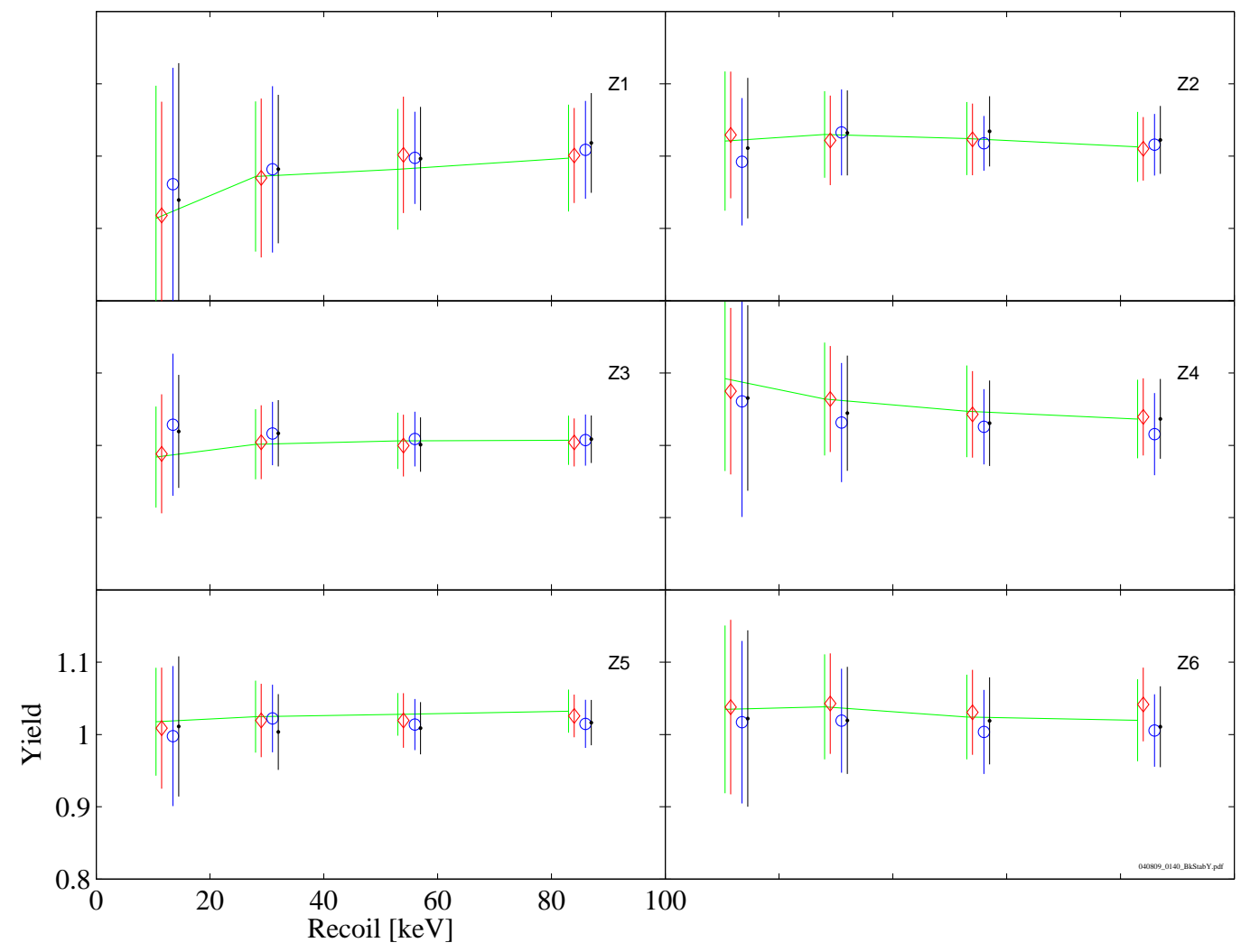

Figure 6.12: Mean and width of events in the Electron Recoil band for four sets of WIMP search data: 10/11-11/1 (green), 11/1-11/20 (red), 11/20-12/15 (blue), and 12/15-1/11 (black). 


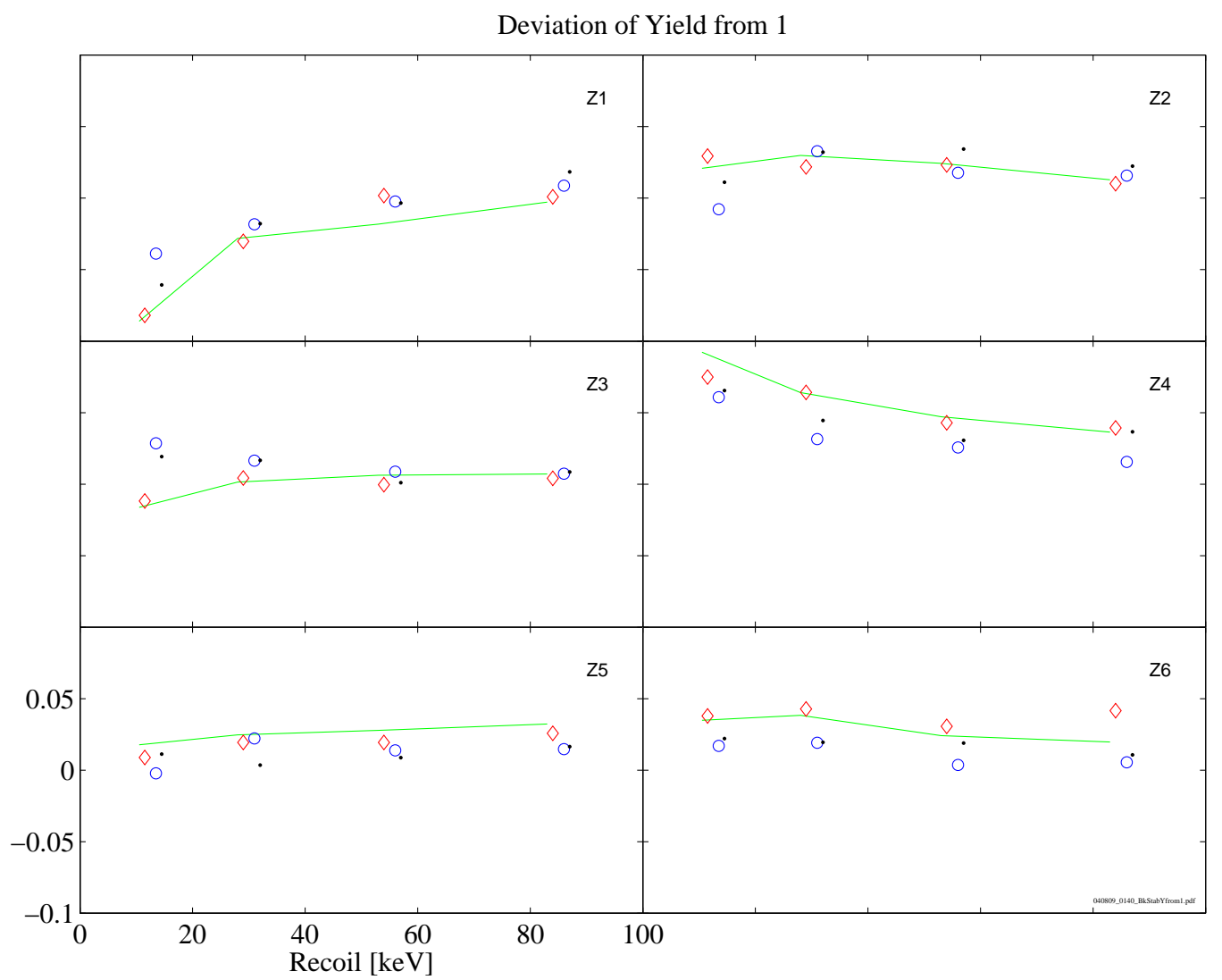

Figure 6.13: Same as Fig. 6.12 only showing the difference of the means from yield $=1$. 10/11-11/1 (green line), 11/1-11/20 (red diamonds), 11/20-12/15 (blue circles), and 12/15-1/11 (black dots). All of the Ge ZIPs deviated from yield $=1$ by less than $5 \%$. 


\subsubsection{Stability of the Cut Efficiencies}

A systematic uncertainty in our analysis arises if the detector performance during the WIMP search data differs substantially from the detector performance during the calibrations that we use to estimate the efficiencies. Since we use the ${ }^{252} \mathrm{Cf}$ calibrations to estimate the efficiencies of the Ionization Yield cut, the Qinner cut, and the Phonon Timing cut, we can estimate the size of this systematic error by comparing the fraction of events passing each cut throughout the WIMP search data to the fraction passing the cuts during the ${ }^{252} \mathrm{Cf}$ calibrations. Since the WIMP search data does not have a significant number of events in the Nuclear Recoil acceptance band, we are limited to using the electron recoils in the Electron Recoil band instead.

- Ionization Yield cut: For the ionization yield cut, we compare the fraction of events in the $2.2 \sigma$ Electron Recoil band that are also in the $2 \sigma$ Electron Recoil band (see Fig. 6.14).

- Qinner cut: For the Qinner cut, we compare the fraction of events in the $2 \sigma$ Electron recoil band that also pass the Qinner cut (see Fig. 6.15).

- Phonon Timing cut: For the Phonon Timing cut, we compare the fraction of events in the $2 \sigma$ Electron Recoil band that also pass the phonon timing cut (see Fig. 6.16).

Figs. 6.14, 6.15, and 6.16 show the pass fractions for each of the three cuts for each detector. Figs. 6.17, 6.18, and 6.19 show the differences in the pass fractions between the WIMP search data and the neutron calibrations. The variations between the WIMP search data and the neutron calibration data are small. We make a conservative estimate of the systematic uncertainty by taking the maximum variation for the energy range above the phonon threshold and below $50 \mathrm{keV}$. For the Ge detectors, all of the variations are less than $3 \%$ with the exception of the ionization yield cut for Z1 (5\%), the timing cut for Z2 (5\%), and the timing cut for Z5 (10\%). If we assume that the errors are independent, we can sum them in quadrature for each detector. Table 6.2 summarizes the numbers. We estimate the total systematic error for the combined Ge ZIPs to be less than $7 \%$. 


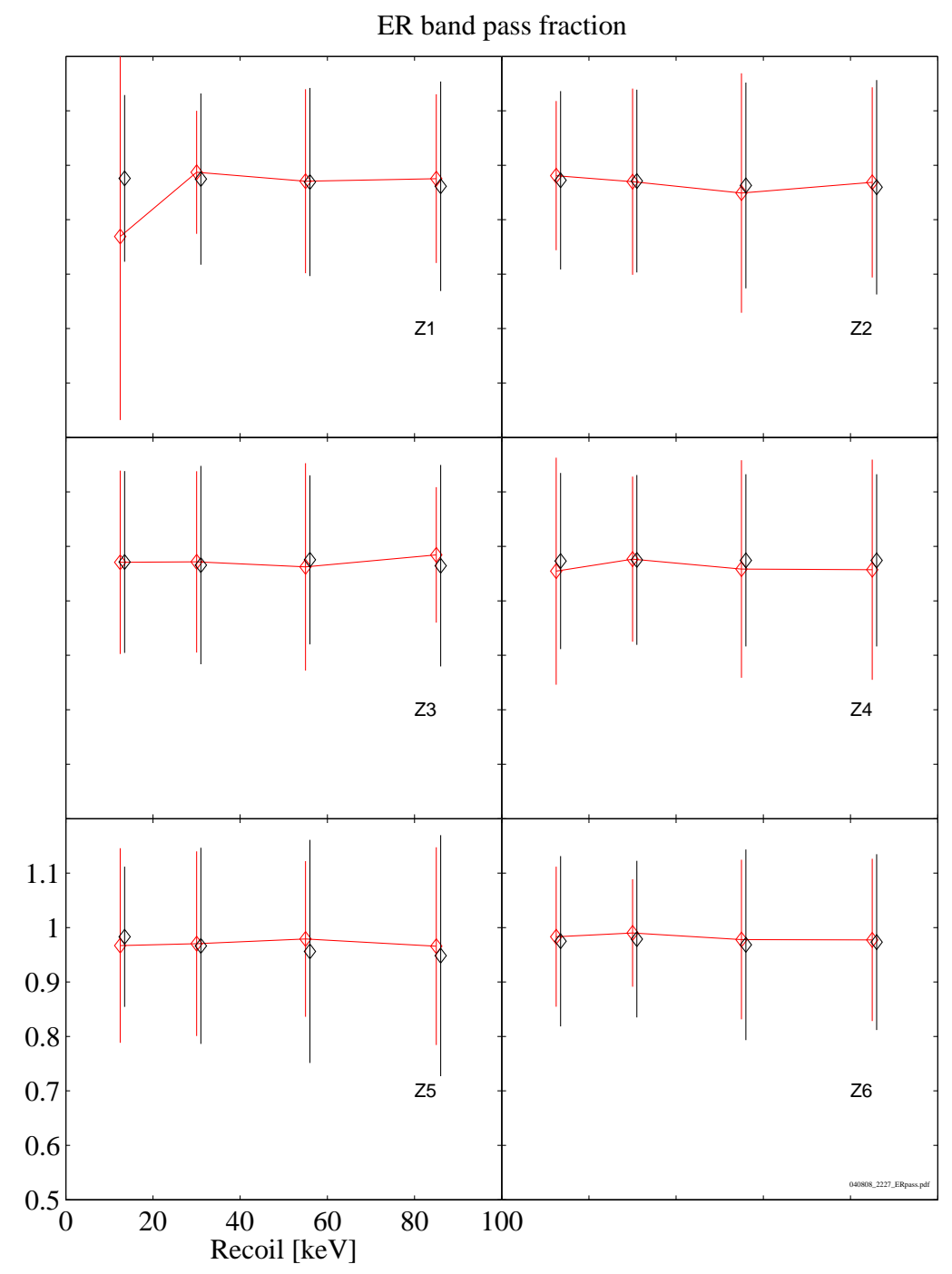

Figure 6.14: Comparison of Electron Recoil band pass fraction between the WIMP search data (black) and the neutron calibrations (red). 


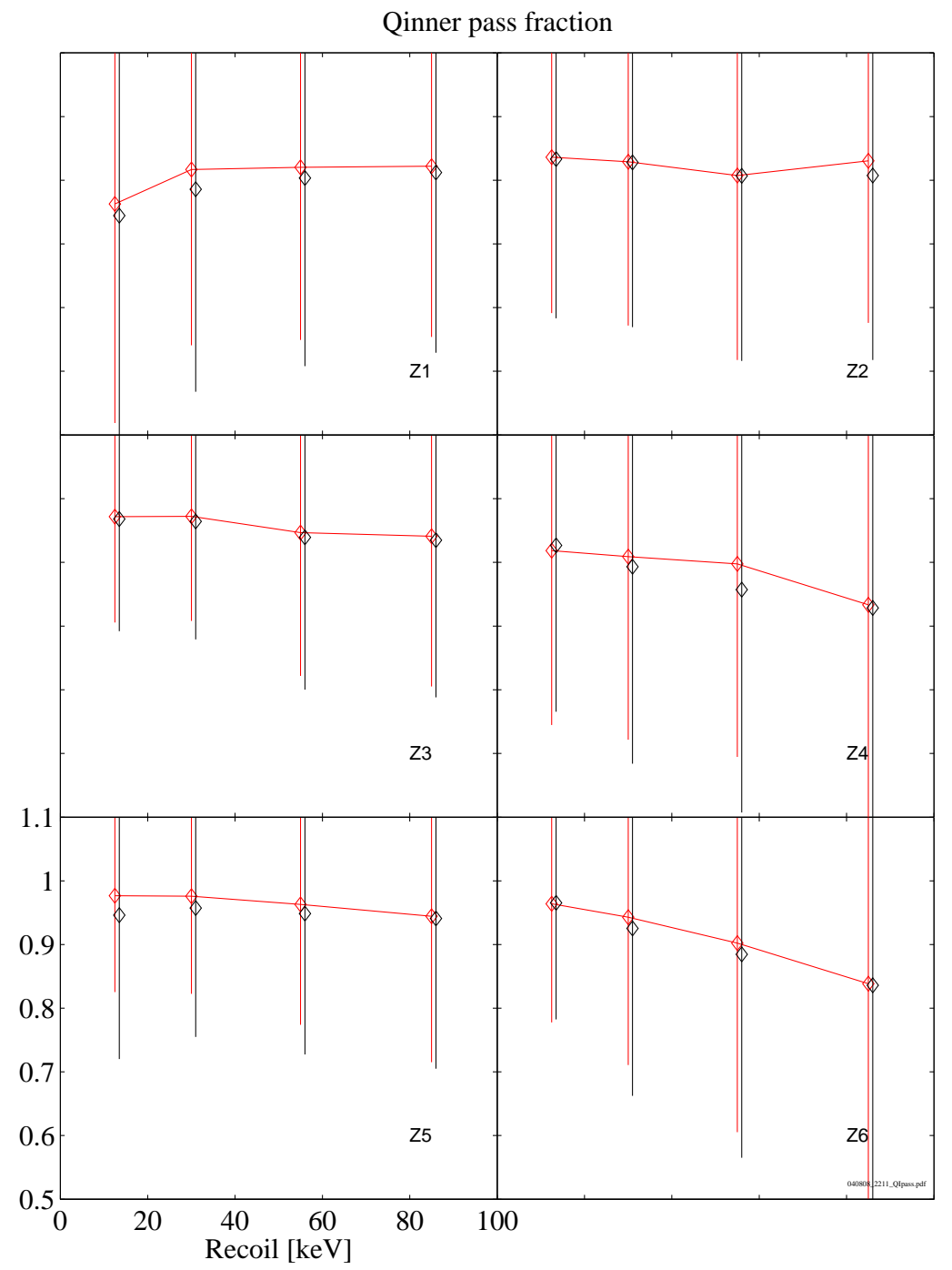

Figure 6.15: Comparison of Qinner cut pass fraction between the WIMP search data (black) and the neutron calibrations (red). 


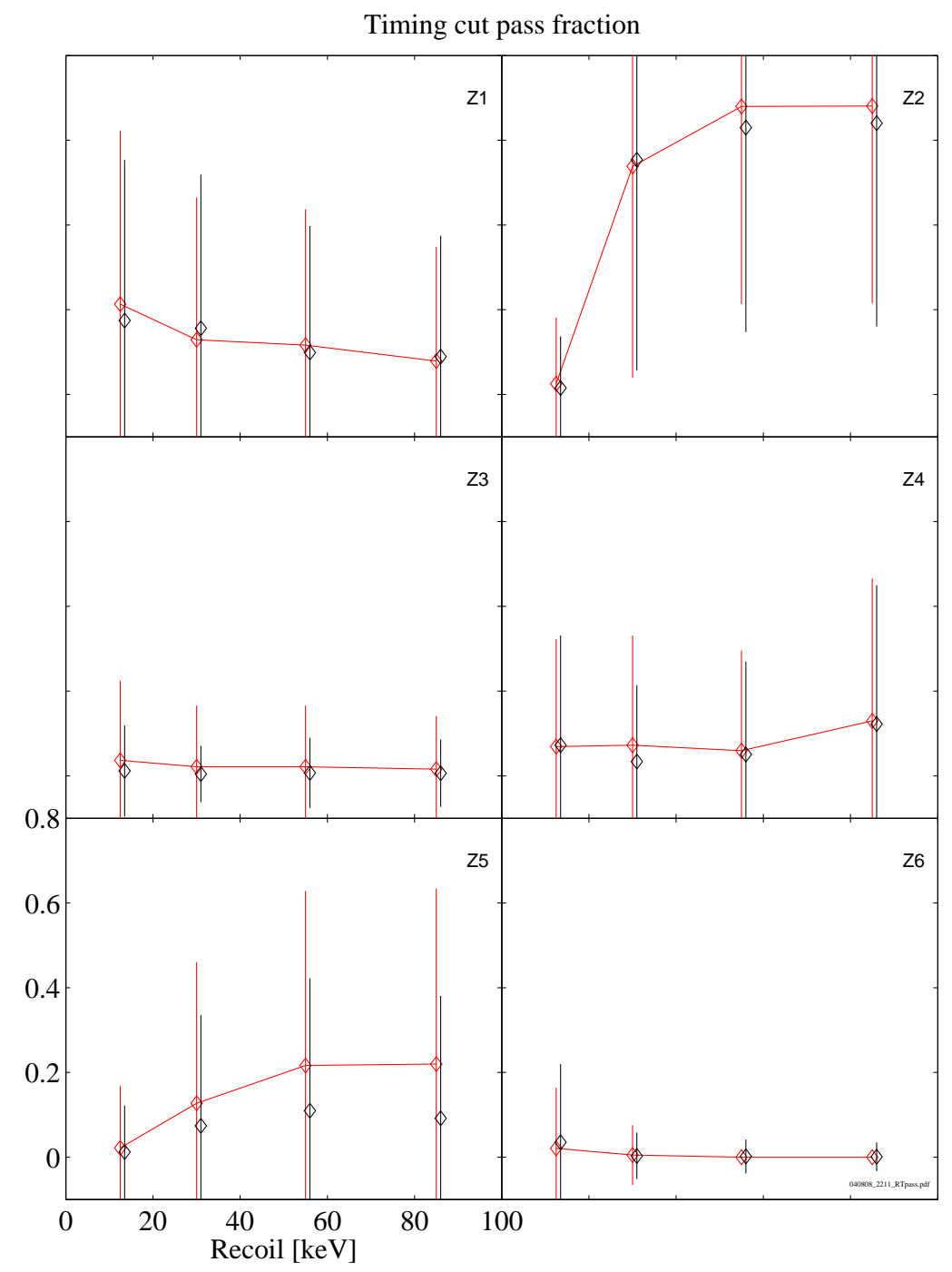

Figure 6.16: Comparison of Phonon Timing cut pass fraction between the WIMP search data (black) and the neutron calibrations (red). 


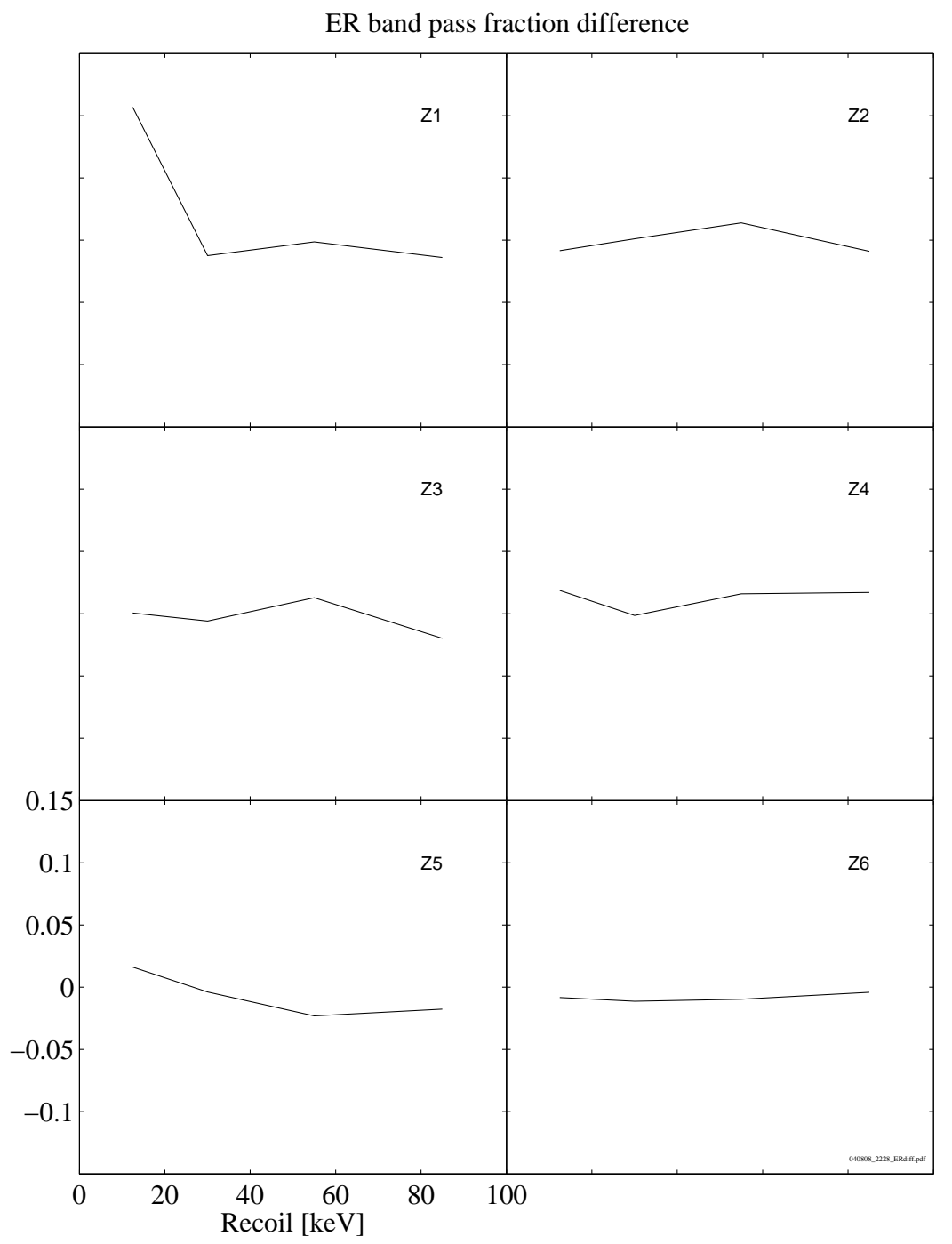

Figure 6.17: Electron Recoil band pass fraction difference between WIMP search data (black) and the neutron calibrations (red). 


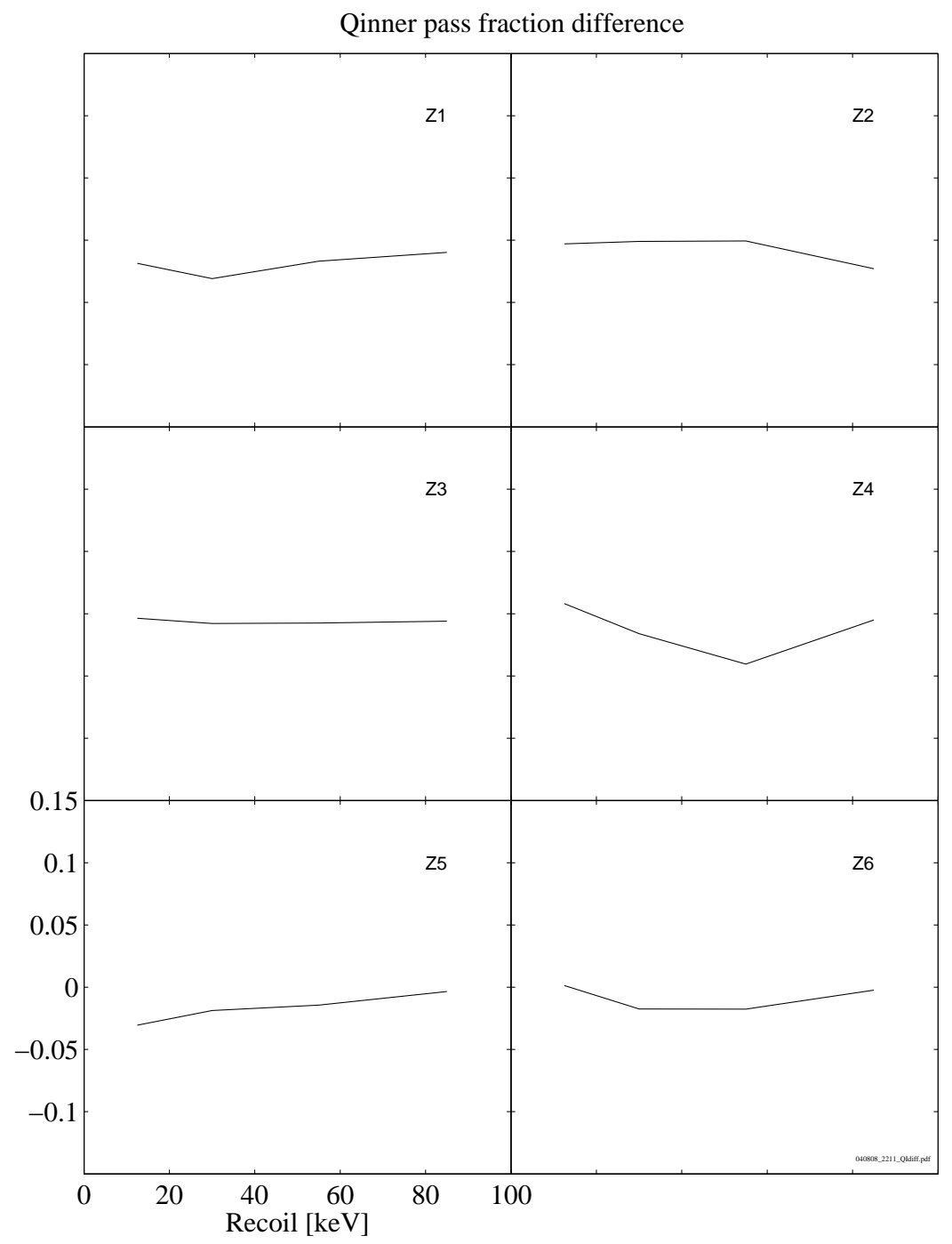

Figure 6.18: Qinner cut pass fraction difference between WIMP search data (black) and the neutron calibrations (red). 


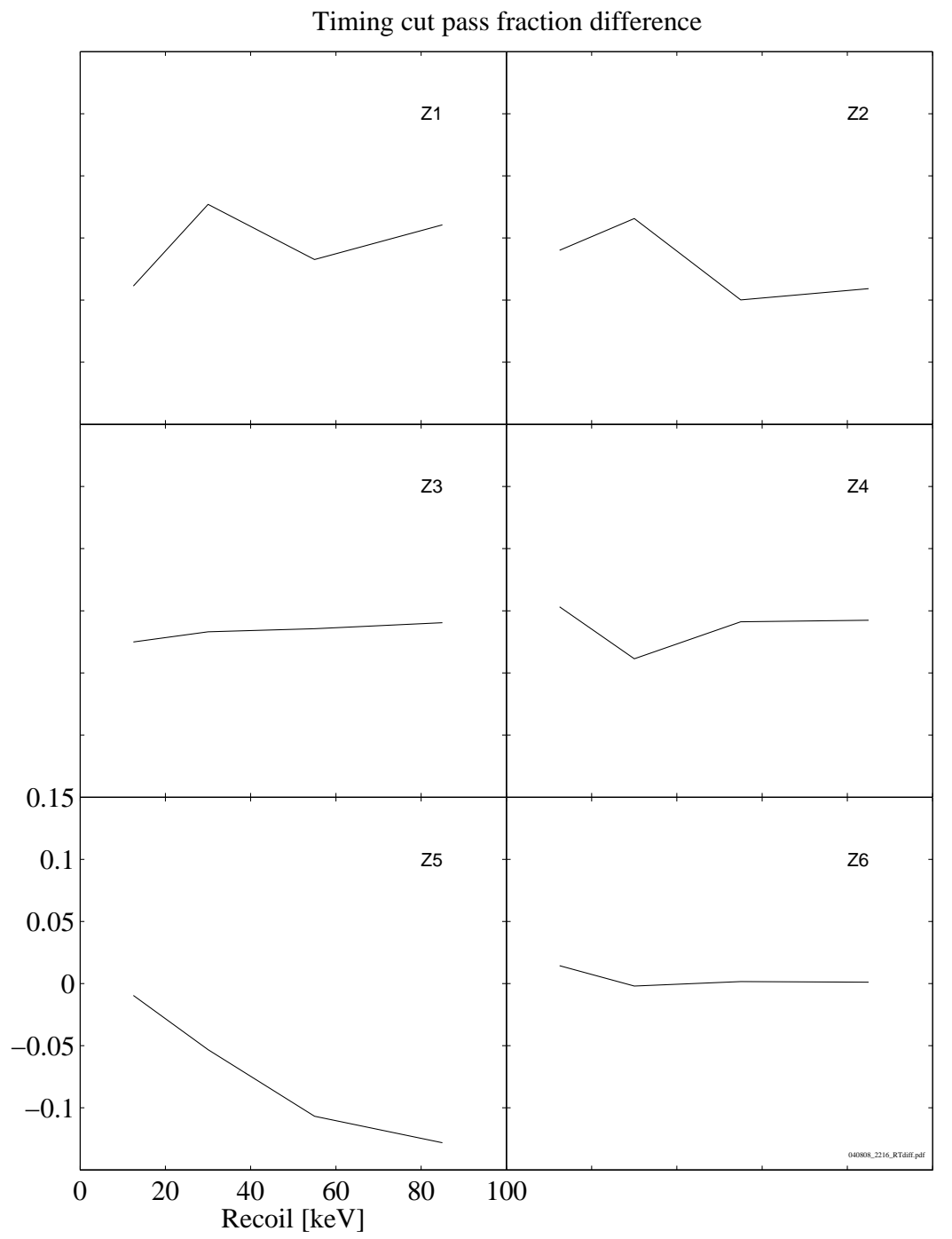

Figure 6.19: Phonon Timing cut pass fraction difference between WIMP search data (black) and the neutron calibrations (red). 


\begin{tabular}{c|c|c|c|c} 
Detector & Yield variation & Qinner variation & Timing variation & Total variation \\
\hline Z1 & $5 \%$ & $3 \%$ & $3 \%$ & $6.56 \%$ \\
Z2 & $3 \%$ & $3 \%$ & $3 \%$ & $6.56 \%$ \\
Z3 & $3 \%$ & $3 \%$ & $3 \%$ & $5.20 \%$ \\
Z4 & $3 \%$ & $4 \%$ & $4 \%$ & $6.40 \%$ \\
Z5 & $3 \%$ & $3 \%$ & $10 \%$ & $10.86 \%$ \\
Z6 & $3 \%$ & $3 \%$ & $3 \%$ & $5.20 \%$
\end{tabular}

Table 6.2: Table summarizing the estimated variation between the background data and the neutron calibration for the ionization yield, qinner, and phonon timing cuts. The values are conservative since they correspond to the maximum deviation between the phonon analysis threshold and $50 \mathrm{keV}$. The total systematic error for the combined Ge ZIPs is less than $7 \%$.

\subsection{Exposure and Expected Sensitivity}

\subsubsection{Calculating the Exposure}

The total time live time for the experiment was 52.6 days. We first calculate the energy dependent efficiency for each detector by combining all of the efficiencies and fitting to a cubic as shown in Fig. 6.20. For each detector, we multiply the efficiency curve by the total live time and the detector mass (250 g for $\mathrm{Ge}$ ) and then sum the exposures to obtain the total energy dependent exposure shown in Fig. 6.21. Only the Ge detectors are summed since the Si detectors have little sensitivity to WIMPs.

\subsubsection{Calculating the Expected Sensitivity}

We calculate the expected sensitivity for the exposure shown in Fig. 6.21 by calculating $90 \%$ exclusion limits for an ensemble of simulated experiments with the expected backgrounds listed in Table 6.1. Fig. 6.22 shows the median of these curves together with a band that includes $50 \%$ of the curves. 


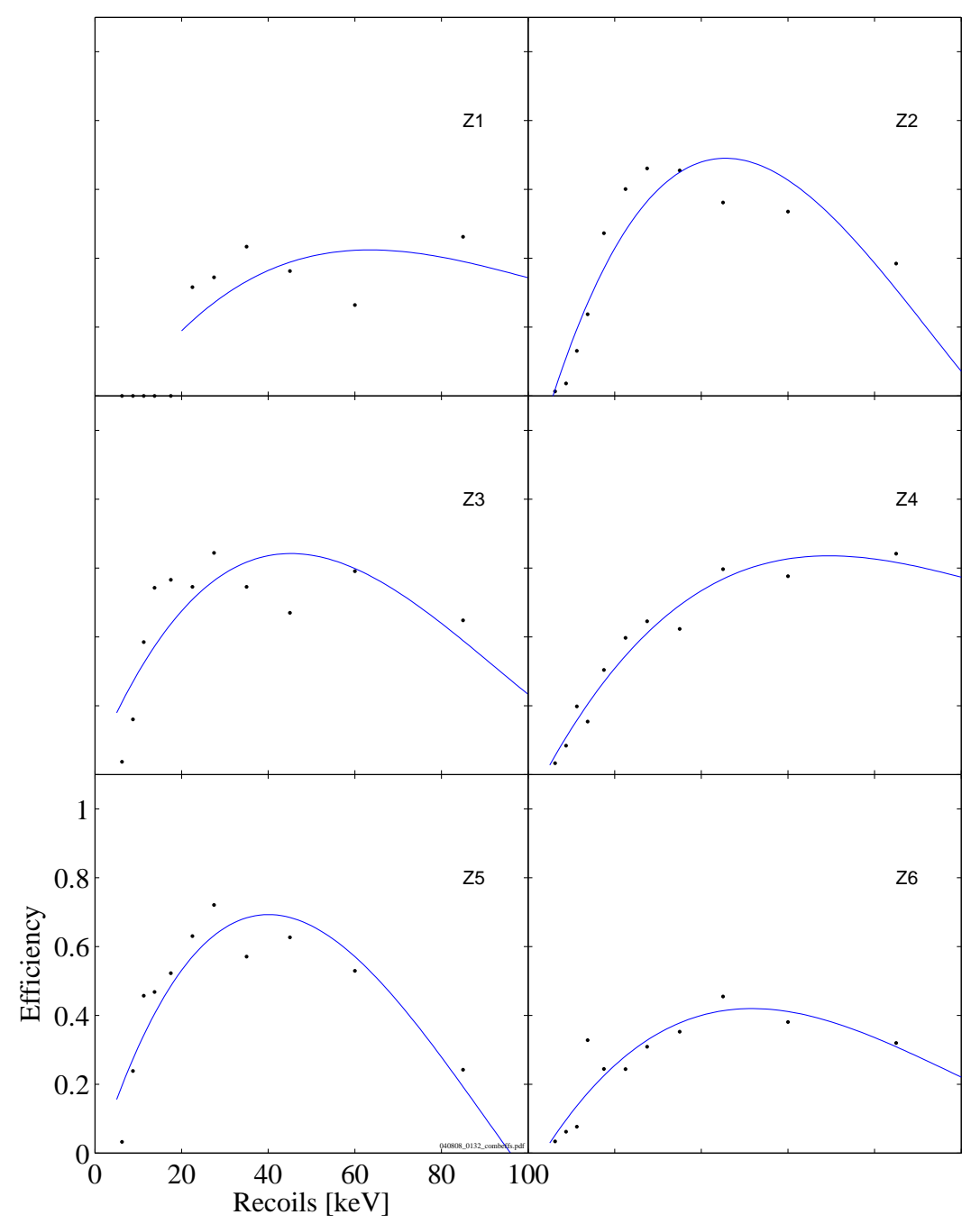

Figure 6.20: Combined efficiencies for the 6 ZIPs. We fit the efficiencies to a cubic. Note that Z1 has an efficiency of zero below its $20 \mathrm{keV}$ analysis threshold while all the other detectors have efficiencies of zero below $5 \mathrm{keV}$. 


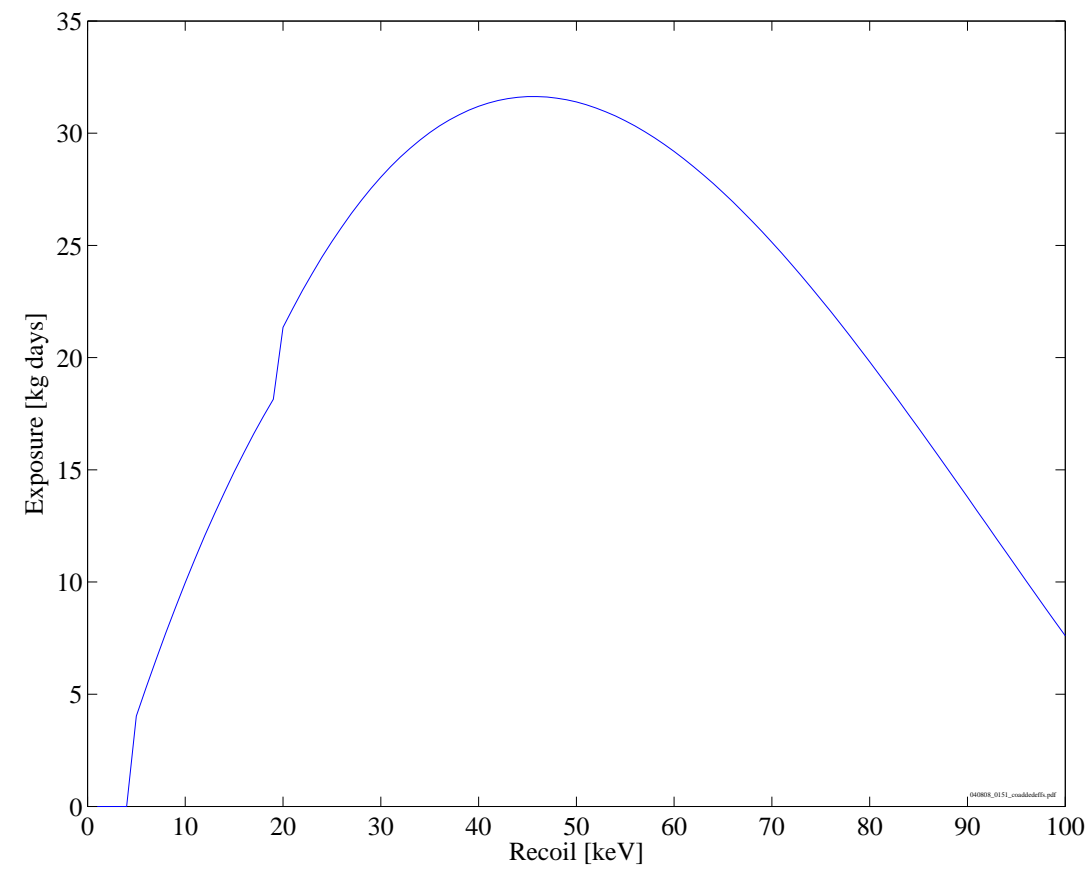

Figure 6.21: Energy dependent exposure in kg days for the summed Ge ZIPs (Z1, Z2, $\mathrm{Z3}, \mathrm{Z5}$ ). The exposure is zero below our analysis threshold of $5 \mathrm{keV}$. The discontinuity at $20 \mathrm{keV}$ corresponds to the $20 \mathrm{keV}$ analysis threshold for Z1. 


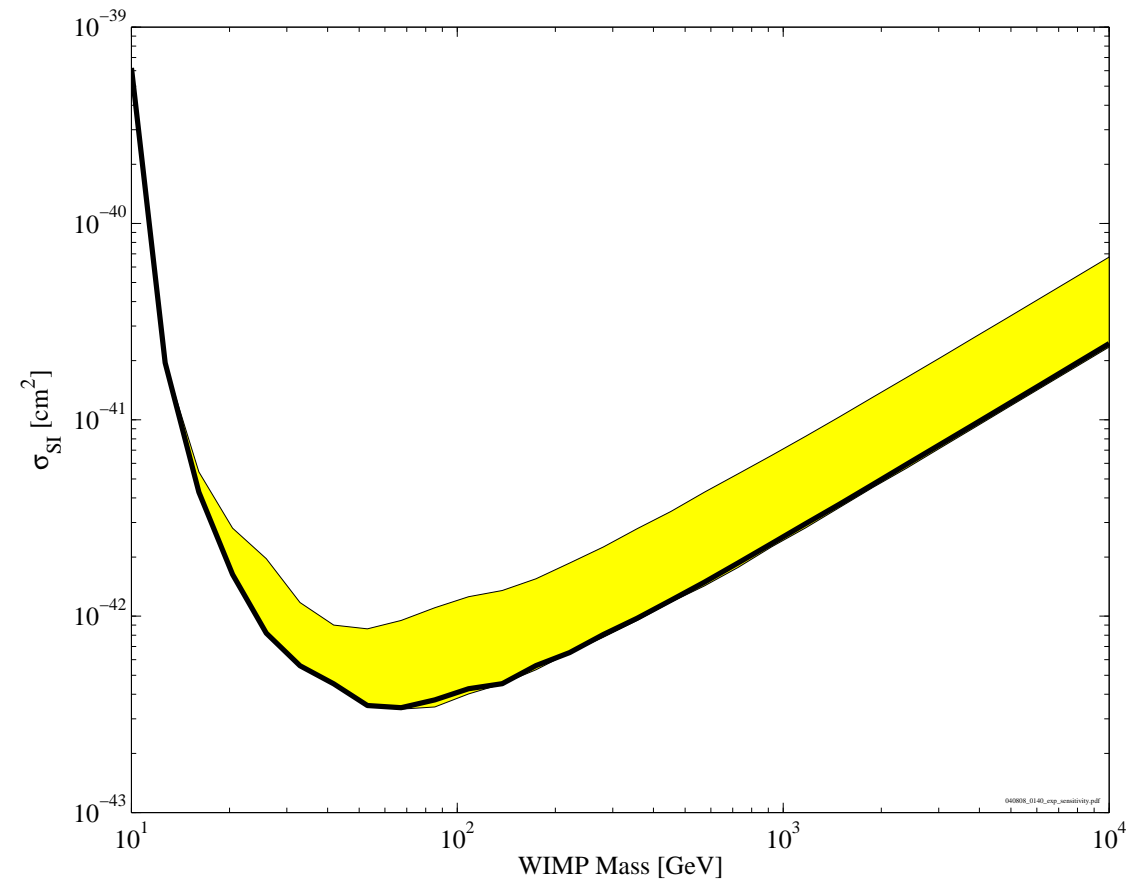

Figure 6.22: Expected sensitivity. 50\% of the simulated experiments lie in the yellow shaded region. The thick black curve corresponds to the median of the ensemble. 


\section{Chapter 7}

\section{Conclusion and Discussion}

\subsection{Results}

The results of the analysis described in Sec. 6.3 are shown in Fig. 7.1 and Table. 7.1. Only one event with a recoil of $64 \mathrm{keV}$ passes all of the cuts. Using the maximum gap method [66], we calculate a curve corresponding to the upper limit on the WIMP nucleon cross section versus mass shown in Fig. 7.2. This limit corresponds to the lower bound of points excluded at $90 \%$ confidence. It is clear from Fig. 7.2 that this limit is consistent with our expected sensitivity.

\begin{tabular}{lc} 
Cut & Number of Events \\
\hline All events & 968,680 \\
Anit-Coincident & 807,419 \\
Single Scatter and Thresholds & 87,596 \\
Low Energy & 13,947 \\
Guard Ring & 8,845 \\
Pile Up & 8,240 \\
Phonon Timing & 1,249 \\
Ionization Yield & 1
\end{tabular}

Table 7.1: Table summarizing the results of applying the cuts described in Sec. 6.3 to the Ge detectors. 


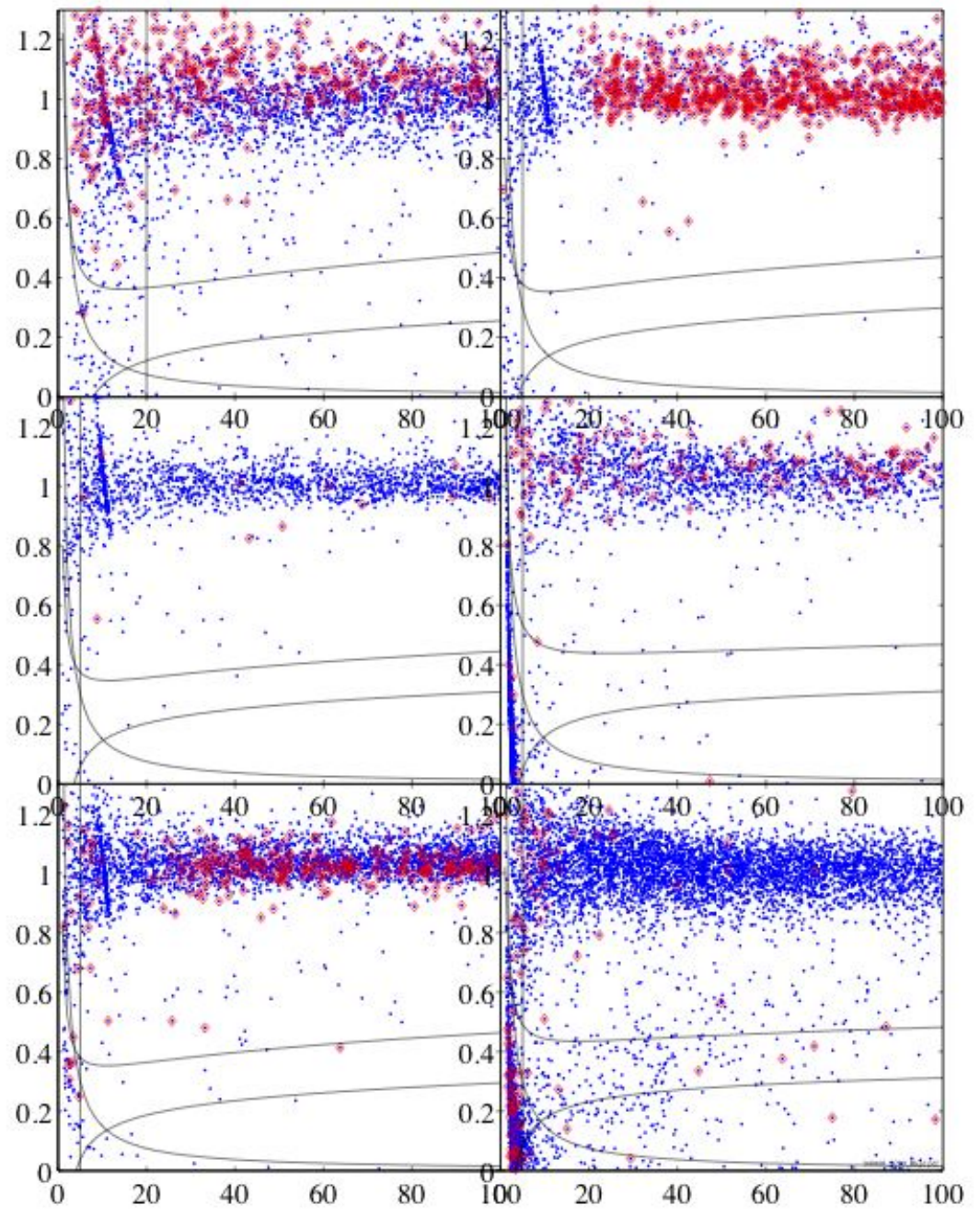

Figure 7.1: Plots for Z1 (top left), Z2 (top right), Z3 (middle left), Z4 (middle right), Z5 (bottom left), and Z6 (bottom right) showing the application of the analysis cuts to the WIMP search data. Blue dots correspond to single scatter inner electrode events after pile up rejection. The red diamonds are events passing the phonon timing cuts. Solid lines indicate the analysis thresholds and the nuclear recoil acceptance bands. Compare with Fig. 6.8. Only one candidate event in Z5 at $\sim 60 \mathrm{keV}$ passes all of the cuts. 


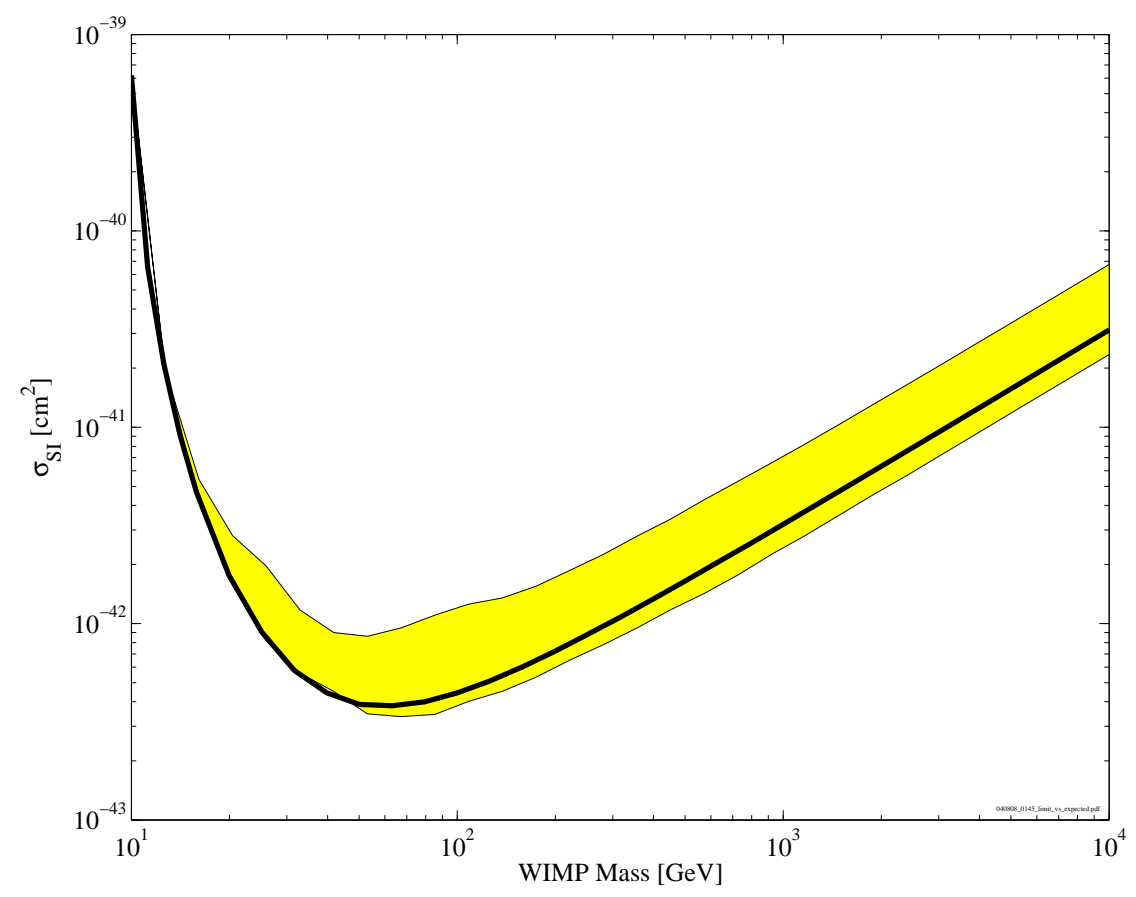

Figure 7.2: Exclusion limit from this analysis. The thick solid line corresponds to the lower bound of points excluded at $90 \%$ confidence. The yellow band corresponds to the region which contains the sensitvity for $50 \%$ of an ensemble of simulated experiments with our expected backgrounds listed in Table 6.1 .

Fig. 7.3 shows an exclusion plot comparing this result with the results of a few other experiments. As of this thesis, the limit from the first CDMS II WIMP search at Soudan is the world's most sensitive limit. It is clear that there is a significant discrepancy between the CDMS II result and the DAMA signal claim. Additionally, the CDMS II result confirms that events seen by CDMS I and EDELWEISS must be neutrons. Fig. 7.4 shows an exclusion plot showing the exculsion curve together with cross section regions predicted by various supersymmetric models. 


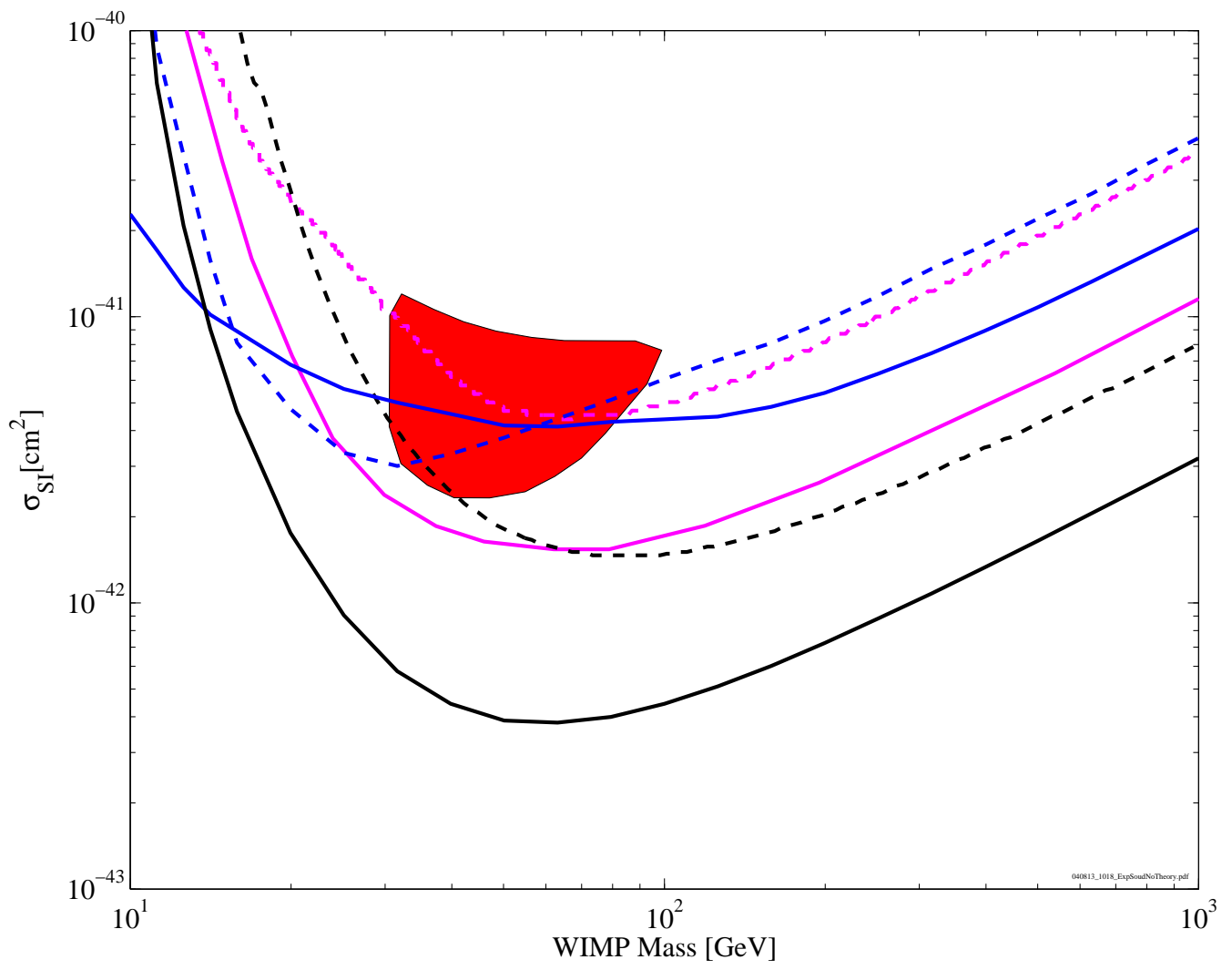

Figure 7.3: Plot of $\sigma_{S I}$ per nucleon versus WIMP mass. Exclusion curves for EDELWEISS (black dashed) [40], CDMS I - SUF (blue dashed) [41, 43], CDMS II - SUF (blue solid) [42, 37], ZEPLIN (magenta solid), CRESST (magenta dashed), and CDMS II - Soudan (black solid). The red region is the DAMA detection claim [39]. Limitplot courtesy of [44]. 


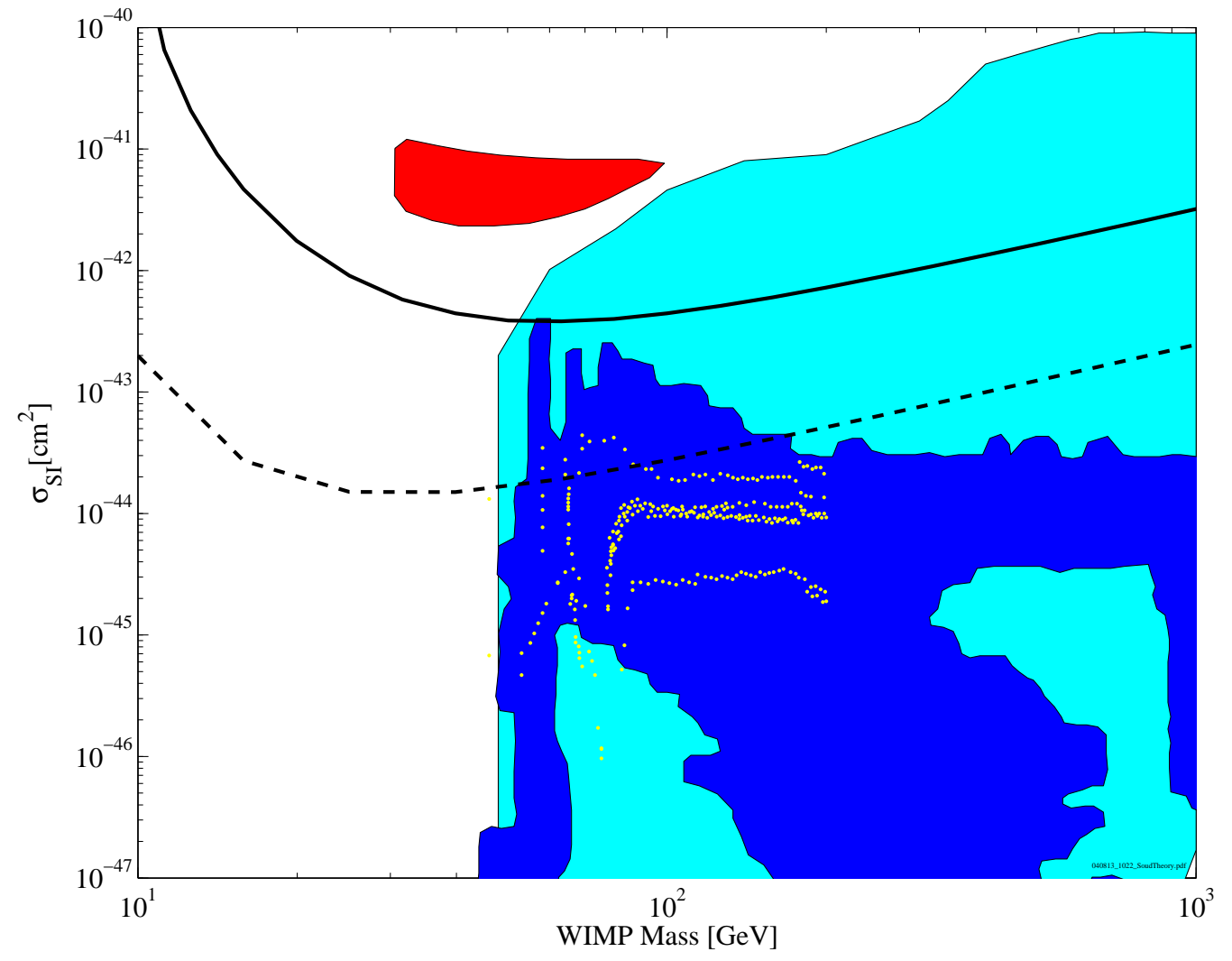

Figure 7.4: Limit plot showing allowed regions corresponding to allowed models from Fig. 1.8[32] (cyan), recent mSUGRA framework calculations using Markov chain monte carlos (dark blue) [67], and split supersymmetry (yellow dots). The black curve is the current Soudan Run 118 limit. The dashed curve is the expected final CDMS II Soudan sensitivity. The red region is the DAMA detection claim [39]. Limitplot courtesy of [44] 


\subsection{The Next Steps...}

\subsubsection{CDMS II}

From Fig. 7.4, it is clear that a significant amount of the neutralino mass-cross section parameter space still remains to be explored. The CDMS collaboration is already making efforts to further the sensitivity of the experiment. Fig. 7.4 also shows the CDMS II Soudan target sensitivity. In order to achieve this goal, developments are being made on two fronts: increasing the WIMP sensitive mass and understanding and rejecting the surface electron recoil background.

\section{Increasing the WIMP Sensitive Mass}

CDMS II has just concluded a second WIMP search run using two Towers of ZIP detectors. This second run (called Run 119) has accumulated over 70 live days of WIMP search data with six 250 g Ge ZIPs. In the Fall of 2004, the collaboration will warm up the fridge and install an additional three Towers to bring the total number of Ge detectors to fifteen.

\section{Understanding and Rejecting the Surface Electron Recoil Background}

For the current CDMS II Soudan experiment, the dominant background comes from electron recoils in the dead layer. This background has two components. One component comes from betas emitted by radioactive contaminants on the detector surface. The second component comes from surface electron recoils caused by the scattering of external photons. Current work on simulations of both contamination betas and ejectrons is underway and looks promising. Combining information from these simulations together with increased in-situ ejectron statistics should make it possible to fully characterize the detector response to surface events.

It is also possible to make improvements in our analysis. V. Mandic [63] and G. Wang [68] have developed additional analysis methods for rejecting surface events. Both methods utilize additional phonon information and significantly improve the beta rejection increasing the sensitivity at lower energies. 


\subsubsection{Beyond CDMS II}

Fig. 7.4 indicates that there is still a significant parameter space that can be explored even after CDMS II has reached its expected sensitivity at Soudan. The CDMS collaboration is already making plans to increase the sensitivity further. Three issues need to be addressed: reduction of the neutron background, reduction and increased rejection of the beta background, and substantial increase of mass in a timely manner.

\section{The Neutron Background}

Just as at the Stanford Underground Facilities, neutrons produced by cosmogenic activity in the rock will comprise an irreducible background for CDMS II. The first results at Soudan demonstrate the effectiveness of going deeper to reduce this background. The CDMS collaboration is considering building another facility at a deeper location such as the Sudbery mine.

\section{The Beta Background}

It is possible, that the irreducible background for CDMS II at Soudan will not be unvetoed cosmogenic neutrons, but will instead be surface electron recoils. CDMS plans to utilize information from a number of beta screening and counting facilities to ensure that future WIMP detectors are as clean as possible. There are also plans to re-engineer the ZIP detectors to make them twice as thick. This would increase the amount of clean Ge. Additionally, these new ZIPs will have a different TES and charge electrode configuration. This new configuration may significantly improve the effectiveness of rejecting events in the dead layer.

\section{Rapid Increase of WIMP Sensitive Mass}

In order to achieve greater sensitivity, CDMS will have to find a way to rapidly increase the mass of the experiment. The development of thicker detectors mentioned earlier will help in accomplishing this. Additionally, there are plans to streamline the detector testing which may result in a substantial increase in the rate of detector production without significant compromise of the detector quality. 


\subsection{Concluding Remarks}

The efforts of the CDMS collaboration in developing the ZIP detector technology and installing the CDMS II experiment in the Soudan Mine have paid off handsomely. The results from the first WIMP search at Soudan are the most sensitive to date by a significant margin.

This upcoming year should be an exciting one as CDMS II makes progress towards its expected sensitivity and probes an unexplored region of parameters. The future looks bright. 


\section{Appendix A}

\section{Instructions and Code for Making Lookup Tables}

\section{A.1 Instructions}

The majority of the effort required for making lookup tables deals with removing outliers from the initial populations of events from which the lookup table is constructed. First, we perform a crude removal of most of the outliers by applying a few simple cuts. These cuts usually involve cuts on Qinner, pileup, recoil, partition, and delay and are coded into make_cuts.m. We shall refer to the events passing the crude cuts as the initial set of events. The next step cuts out the remaining outliers by exploiting the fact that the outliers should be "far away" from the bulk of the event distribution. However, the set of events remaining after application of the crude cuts is too large with the majority of the outliers having a lot of events close by. The solution is carried out in two steps. First, we take a smaller $(\sim 20 \mathrm{k})$ sample of the initial set of events which we call the subset. We then use calc_norm_dist.m with $O(20)$ neighbors to generate the parameters avg_dist and normdist. These values correspond to the average distance to the neighbors and the average of the neighbors' avg_dist. We then impose a cut on normdist and avg_dist to remove the remaining outliers in the subset. The cut usually passes $90 \%$ of the events in the subset. We then use the outlier free subset together with flesh_out_table.m to remove the outliers from the initial set of 
events. The function flesh_out_table.m rejects events in the initial set that are "too far" from the outlier free subset. The final results is a set of events that should be free of outliers. We refer to this set of events as the raw events. The lookup table is constructed from the raw events using make_table.m and the appropriate number of nearest neighbors.

\section{A.2 Code for Selecting Good Events}

This section contains the code for routines that are used in removing outliers.

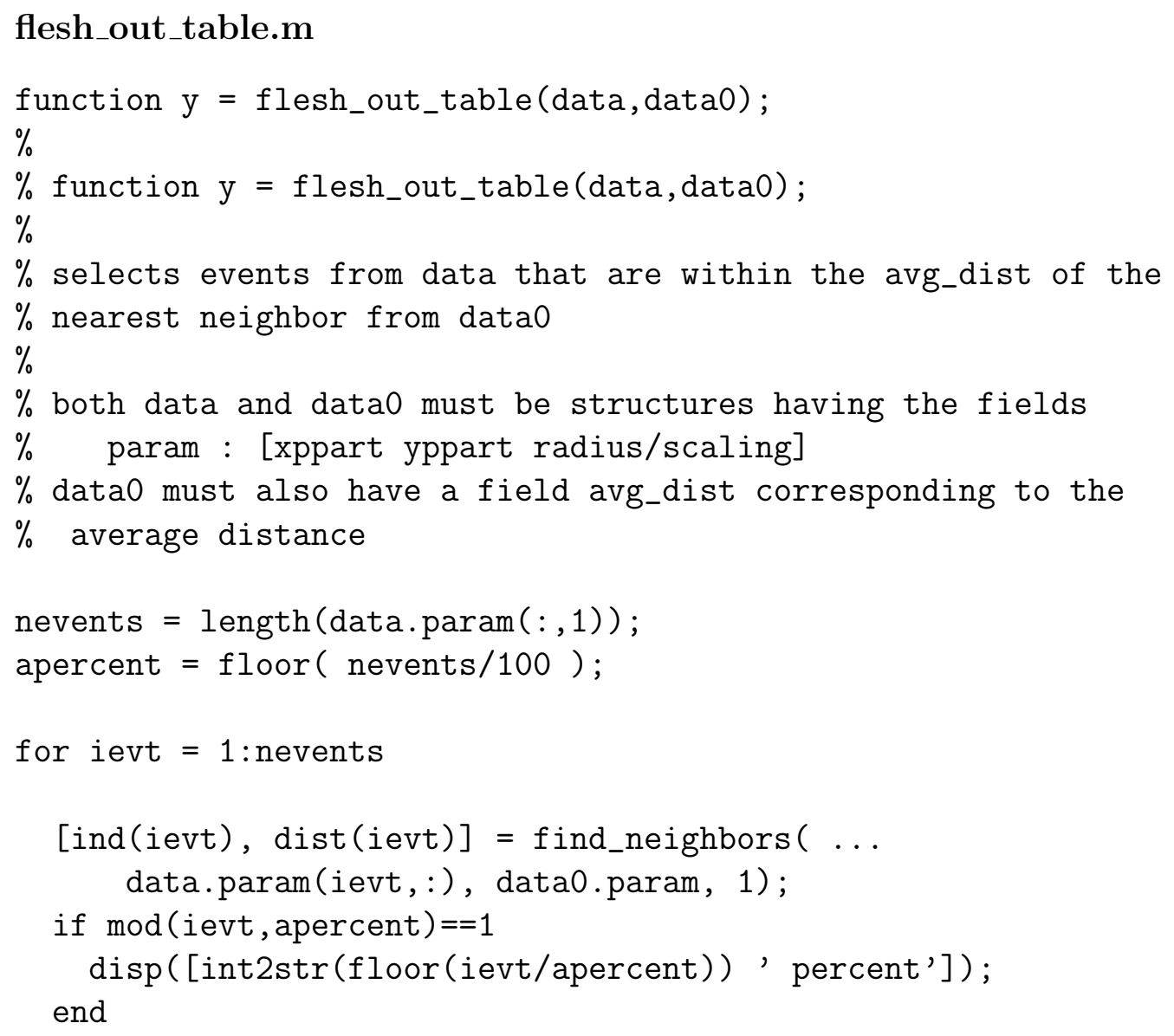


end

cut = bool ( dist <= data0.avg_dist(ind)' );

$\mathrm{y}=\operatorname{assignfields}($ data, cut);

\section{make_cuts.m}

function $[y$, cut $]=$ make_cuts $(\operatorname{detnum})$;

$\%$

$\%$ function $[y$, cut $]=$ make_cuts (detnum);

$\%$ applies cuts to the current CAP session returning a structure

$\%$ with the following fields :

$\%$

$\% q q=$ qsum

$\% \mathrm{rt}=\mathrm{ptrt}$

$\%$ param $=[$ xppart yppart radius/scaling $]$ basically the

$\% \quad$ position vector

$\%$ delay $=[$ xdel ydel $] /$ scaling

$\% \mathrm{pp}=\mathrm{prg}$

$\%$ minrt $=10-40$ risetime of primary channel

$\%$ pdel = delay (relative to QIst) of primary channel

$\%$ minrt4070 $=40-70$ risetime of primary channel

$\%$ pfrac $=\mathrm{px} . / \mathrm{py}$ where $\mathrm{x}$ is the primary channel and

$\% \quad y$ is the cross channel

$\%$ pmaxi $=$ index of primary channel ( $1=\mathrm{A} \quad 2=\mathrm{B} \quad 3=\mathrm{C} \quad 4=\mathrm{D})$

$\%$

$\%$ cuts applied are :

$\%$

$\%$ inrange (prg (detnum, 0$), 0,250$ )

$\%$ inrange (qsum, 10,300) in all detectors

$\%$ cQin

$\%$ cChiSq

$\%$ no glitch

$\%$ no betas

$\%$ apply the cuts

$\%$ have reasonable energy in all detectors

$\operatorname{ct300}=\operatorname{bool}($ ones $(\operatorname{size}($ EventNumber $(401,0)))$ ); 


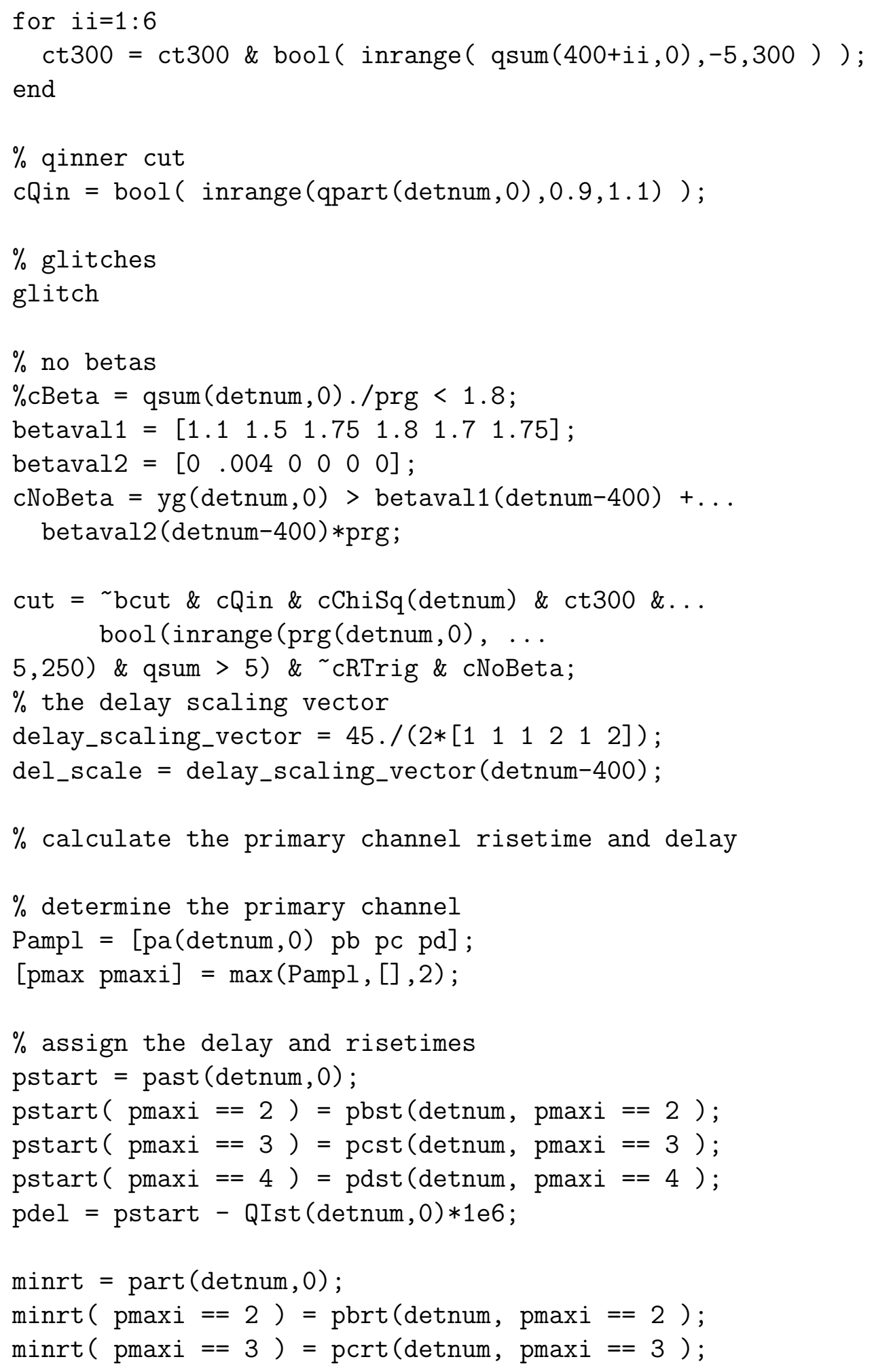




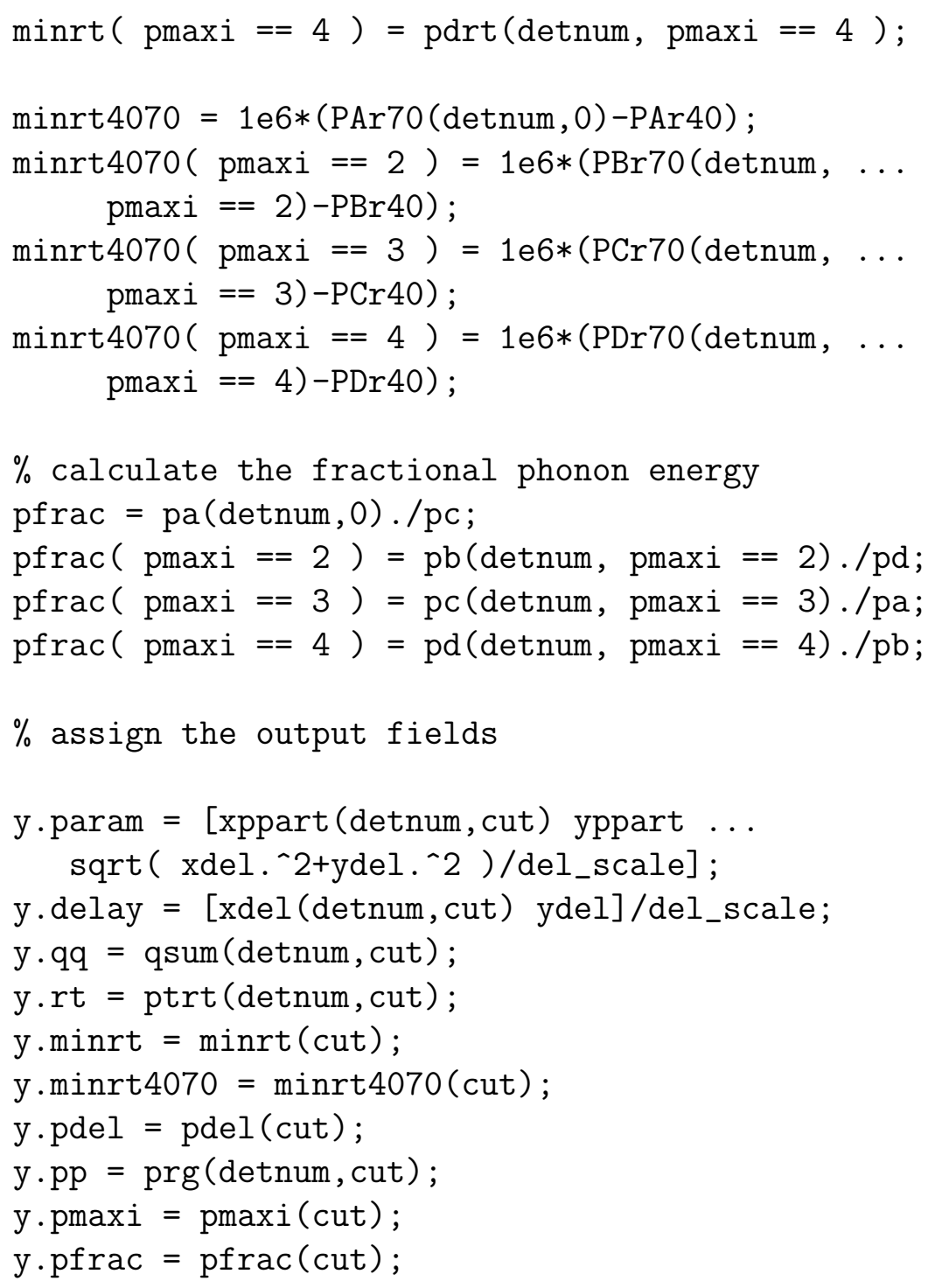

\section{calc_norm_dist.m}

function $\mathrm{y}=$ calc_norm_dist (numNeighbors,data)

$\%$ function $y=$ calc_norm_dist (numNeighbors,data)

$\%$

$\%$ generates a file for the nearest neighbor look up table $\%$

$\%$ inputs :

$\%$ numNeighbors : number of nearest neighbors

$\%$ data : structure of events with the following: 


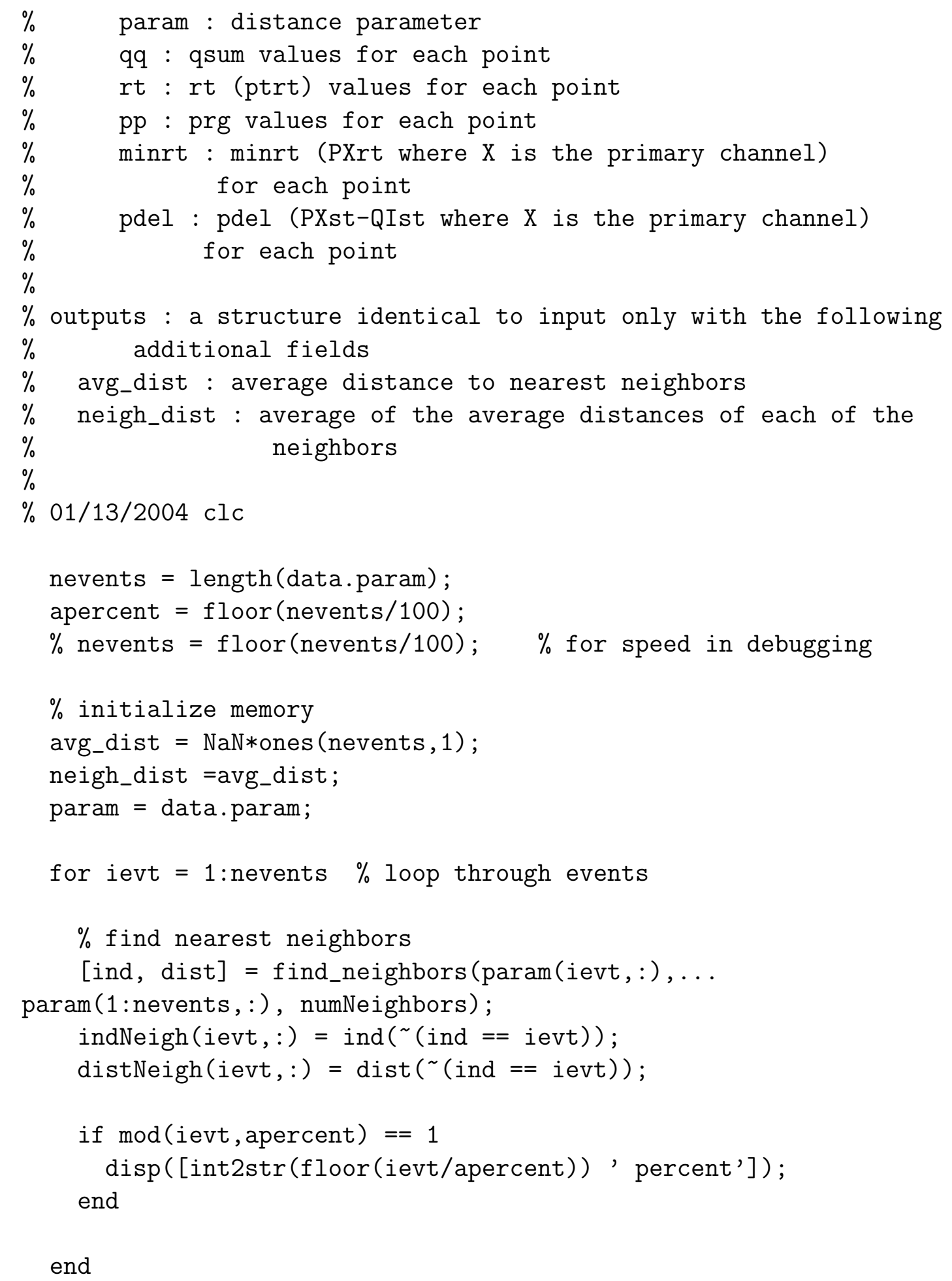




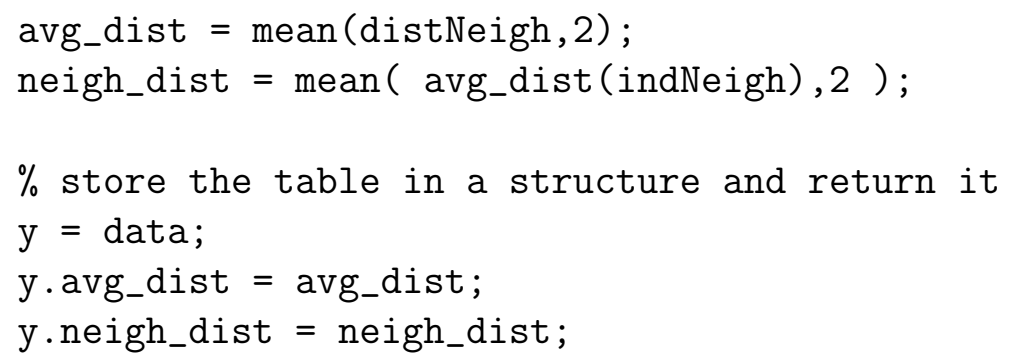

\section{A.3 Code for Making the Table}

This section contains the code for making a table from the raw events.

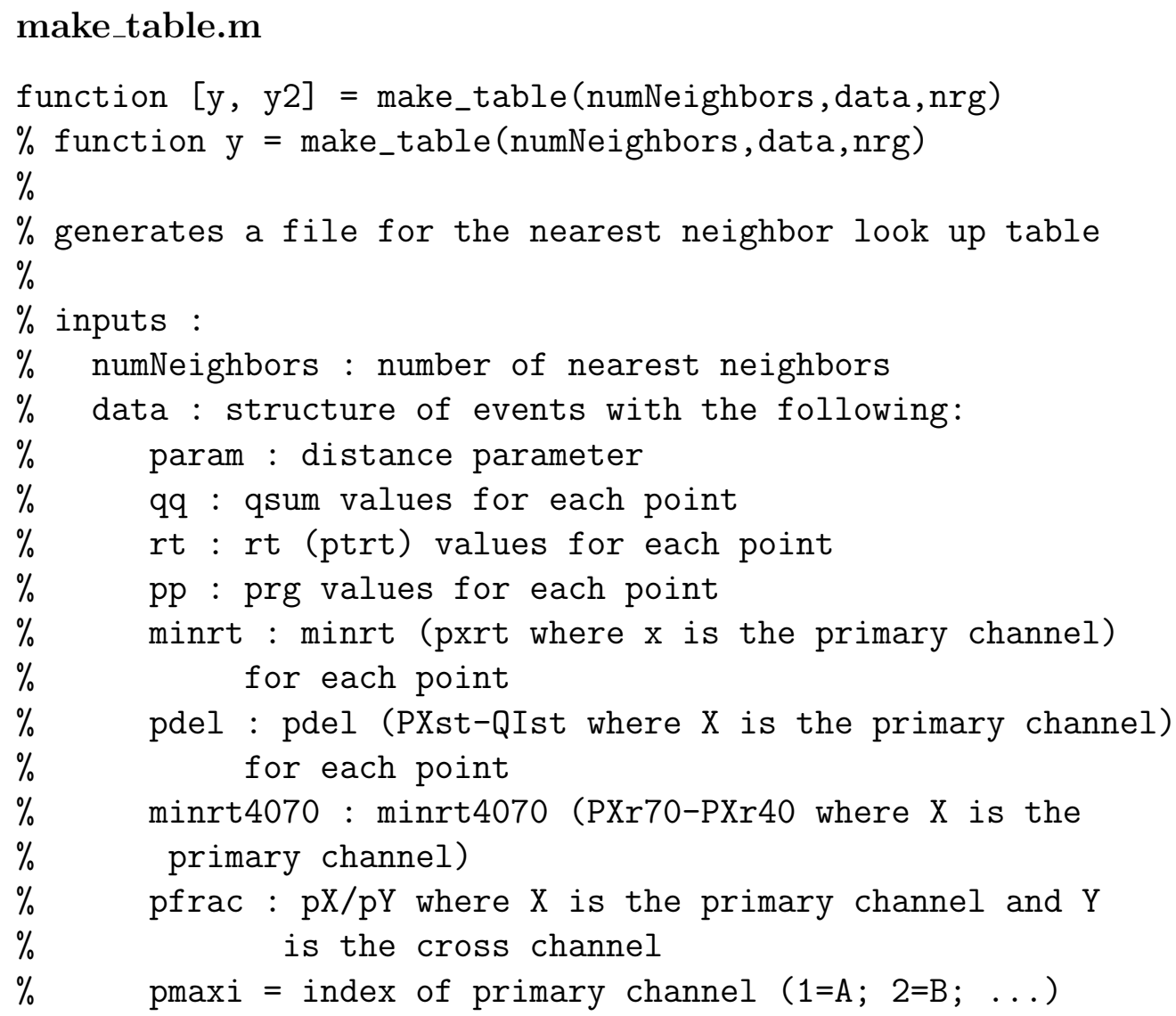




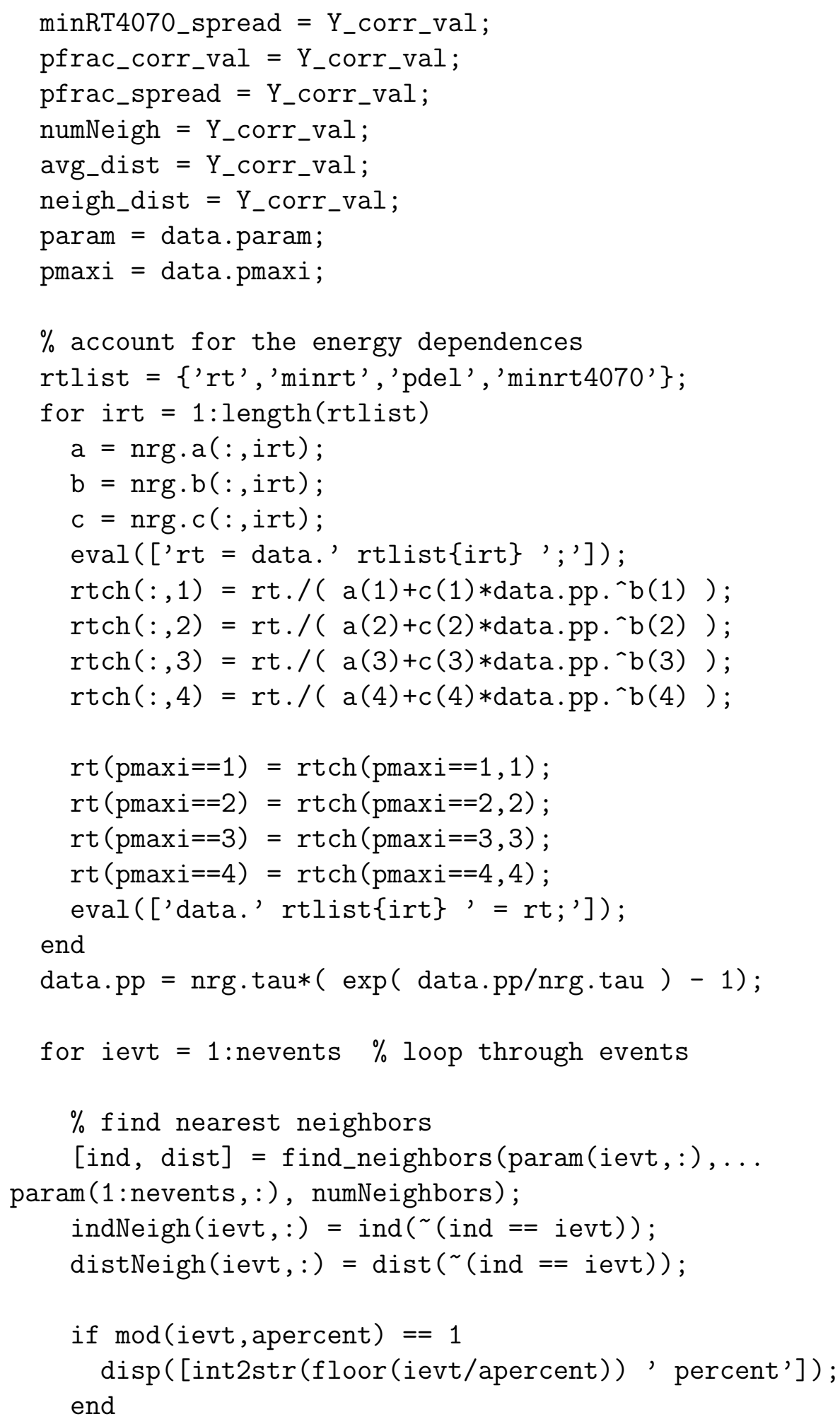


end

$\%$ generate the correction values and spreads

$Y_{-}$corr_val $=$mean $($data.qq (indNeigh)./data.pp (indNeigh) ,2 );

Y_spread $=\operatorname{std}($ data.qq(indNeigh)./data.pp (indNeigh),$\ldots$

[],2 )./ Y_corr_val;

RT_corr_val = mean ( data.rt (indNeigh),2 );

RT_spread $=\operatorname{std}($ data.rt $($ indNeigh $),[], 2)$./RT_corr_val;

minRT_corr_val $=$ mean ( data.minrt (indNeigh), 2$)$;

minRT_spread $=\operatorname{std}($ data $\cdot \min r t($ indNeigh $),[], 2 \ldots$

)./minRT_corr_val;

pdel_corr_val $=$ mean $($ data $\cdot \operatorname{pdel}($ indNeigh $), 2$ );

pdel_spread $=\operatorname{std}($ data $\cdot \operatorname{pdel}($ indNeigh $),[], 2 \ldots$

)./pdel_corr_val;

minRT4070_corr_val $=\operatorname{mean}($ data $\cdot \operatorname{minrt4070}($ indNeigh), 2 );

minRT4070_spread $=\operatorname{std}($ data.minrt4070(indNeigh), [],2 . .

)./ minRT4070_corr_val;

pfrac_corr_val = mean ( data.pfrac(indNeigh),2 );

pfrac_spread = std (data.pfrac(indNeigh), [],2 ...

)./pfrac_corr_val;

avg_dist $=$ mean (distNeigh, 2$)$;

neigh_dist $=$ mean $($ avg_dist (indNeigh),2 );

$\%$ store the table in a structure and return it

$\mathrm{y} \cdot \mathrm{nrg}=\mathrm{nrg}$;

$\mathrm{y} \cdot \mathrm{Y}_{-}$corr_val $=\mathrm{Y}_{-}$corr_val;

$\mathrm{y}$.Yspread $=$ Y_spread;

$\mathrm{y} \cdot \mathrm{RT}_{\text {_corr_val }}=\mathrm{RT}$ _corr_val;

$\mathrm{y} \cdot$ rtspread $=$ RT_spread;

$\mathrm{y} \cdot \mathrm{minRT}$ _corr_val $=$ minRT_corr_val;

$\mathrm{y} \cdot \mathrm{minRT}$ _spread $=$ minRT_spread;

$\mathrm{y} \cdot \mathrm{pdel}$ corr_val $_{\text {c }}$ pdel_corr_val;

$\mathrm{y} \cdot \mathrm{pdel}$ _sptread $=$ pdel_spread; 


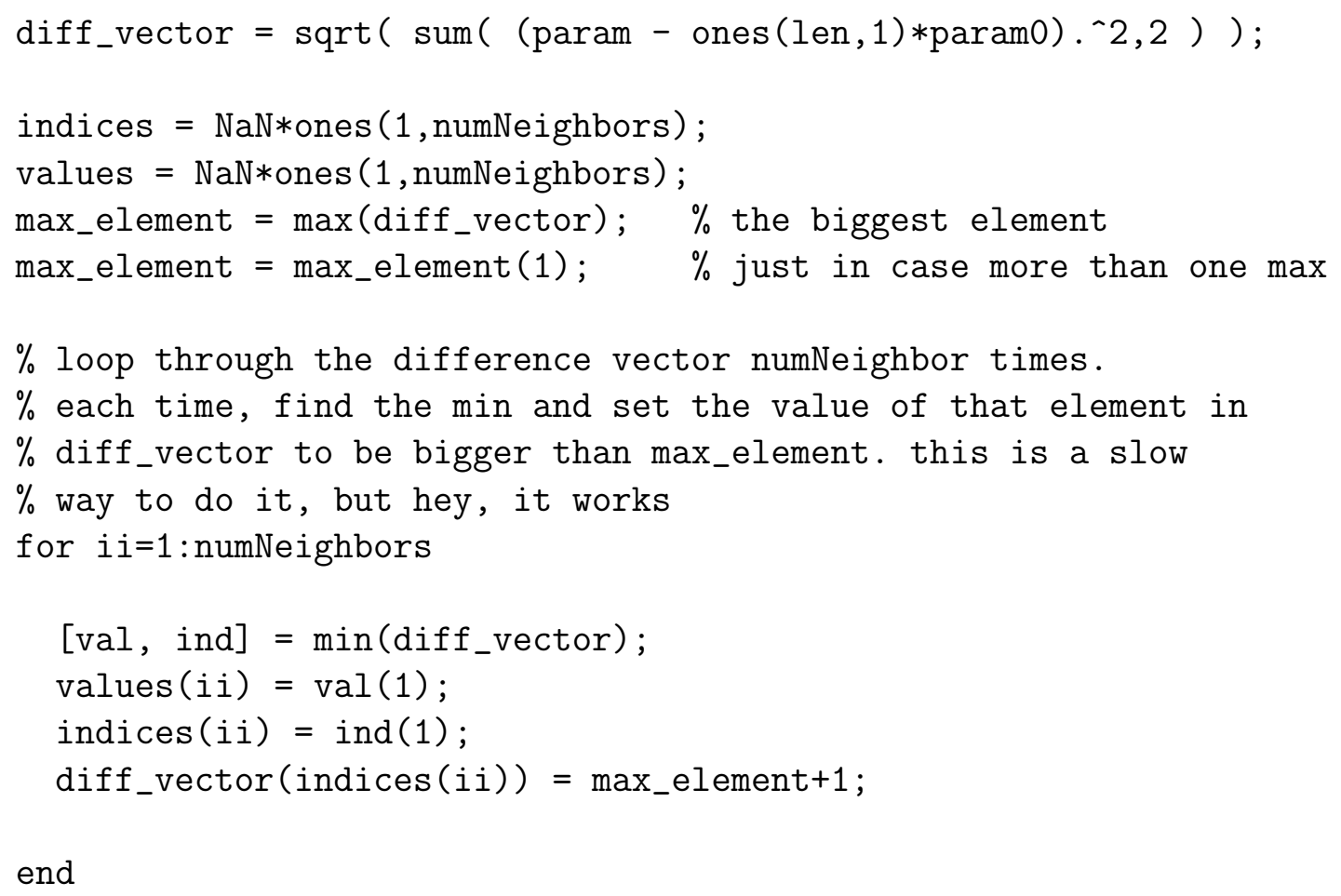

\section{A.4 Code for Some Useful Table Utilities}

This section contains the code for some routines that are useful for manipulating the table. In particular, the routine, manifoldmovie.m, generates movies for 3D visualization of the events.

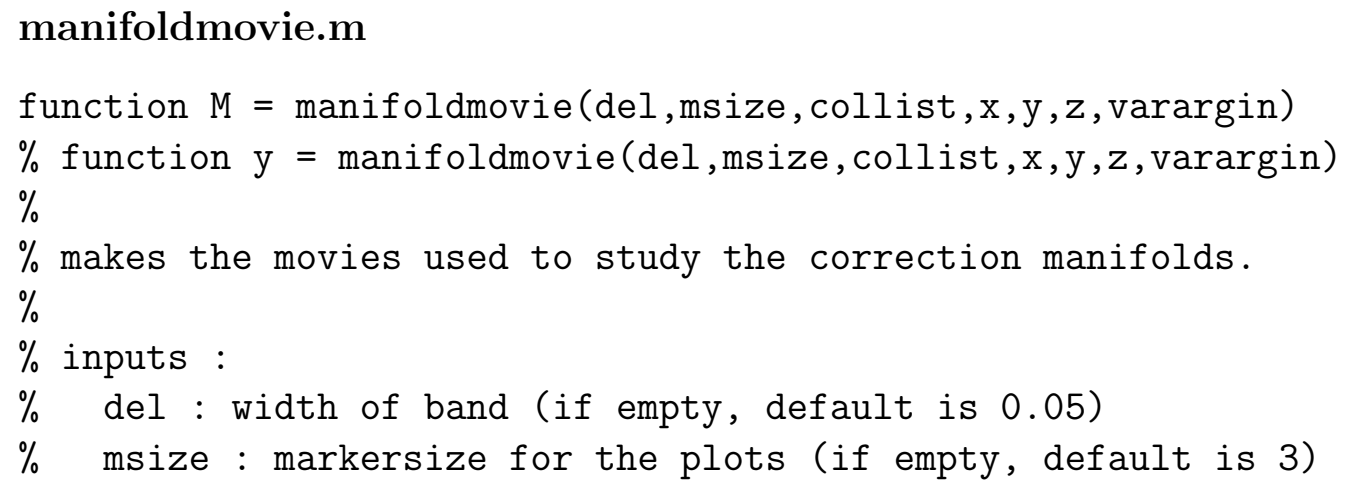




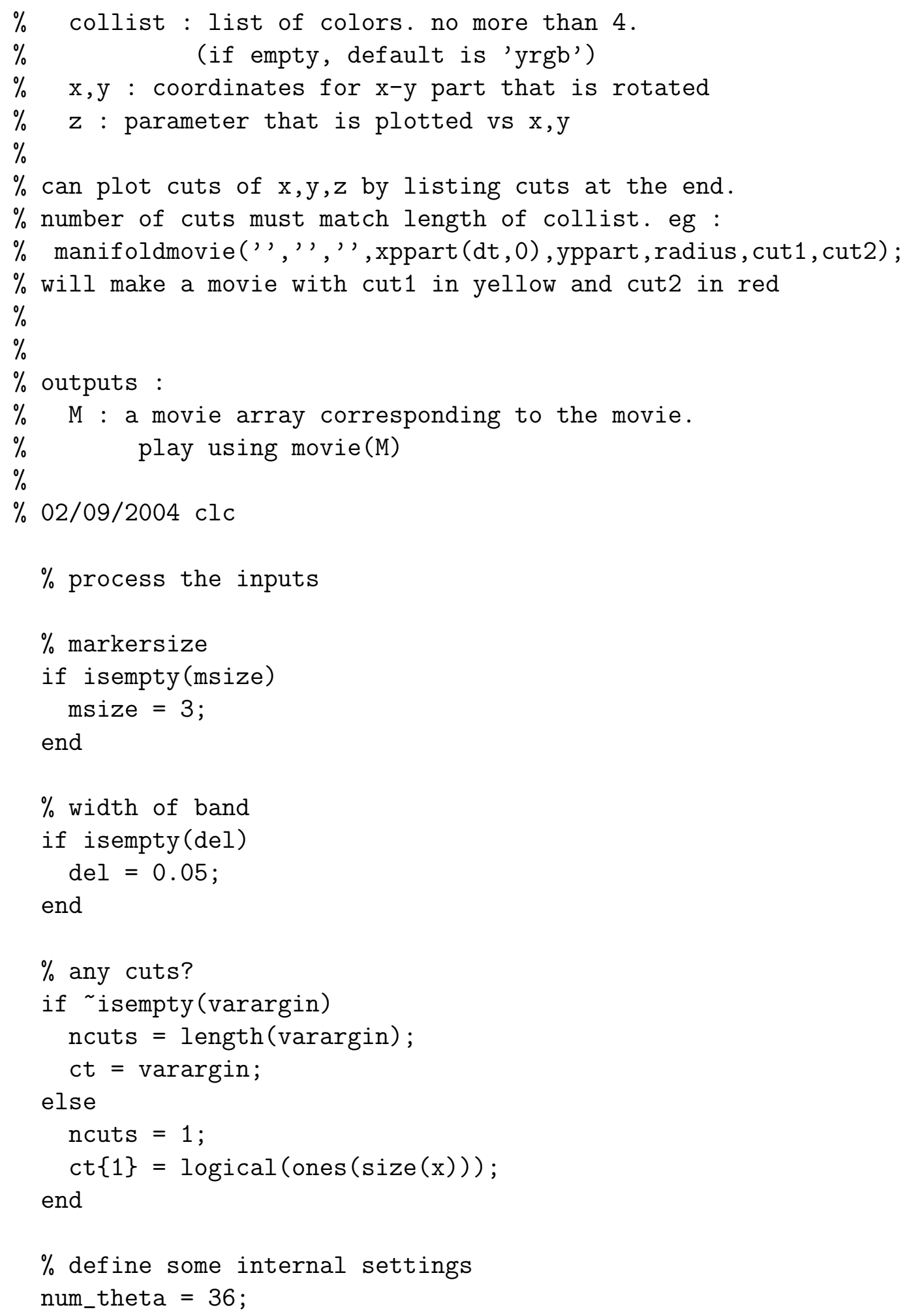




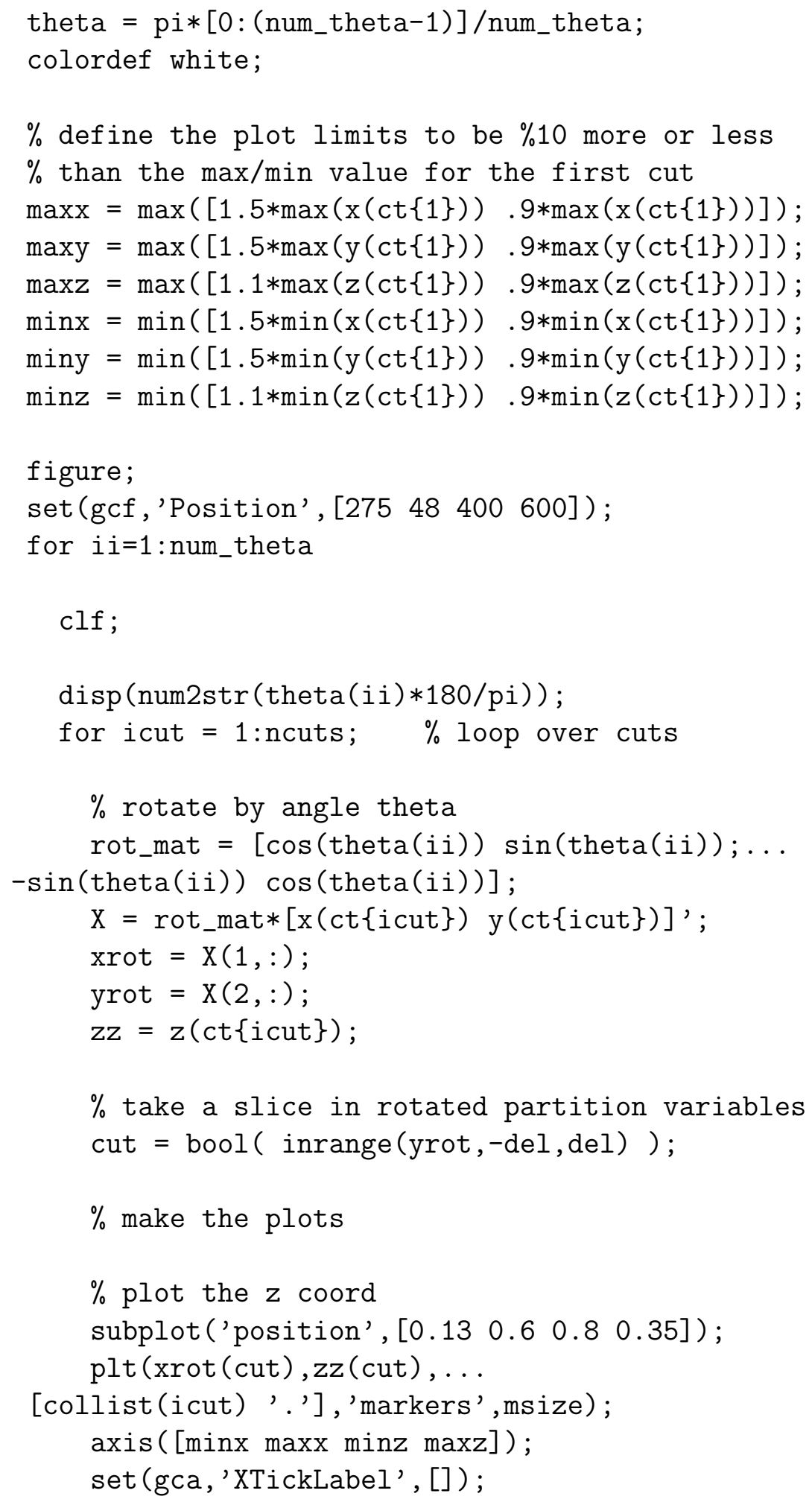




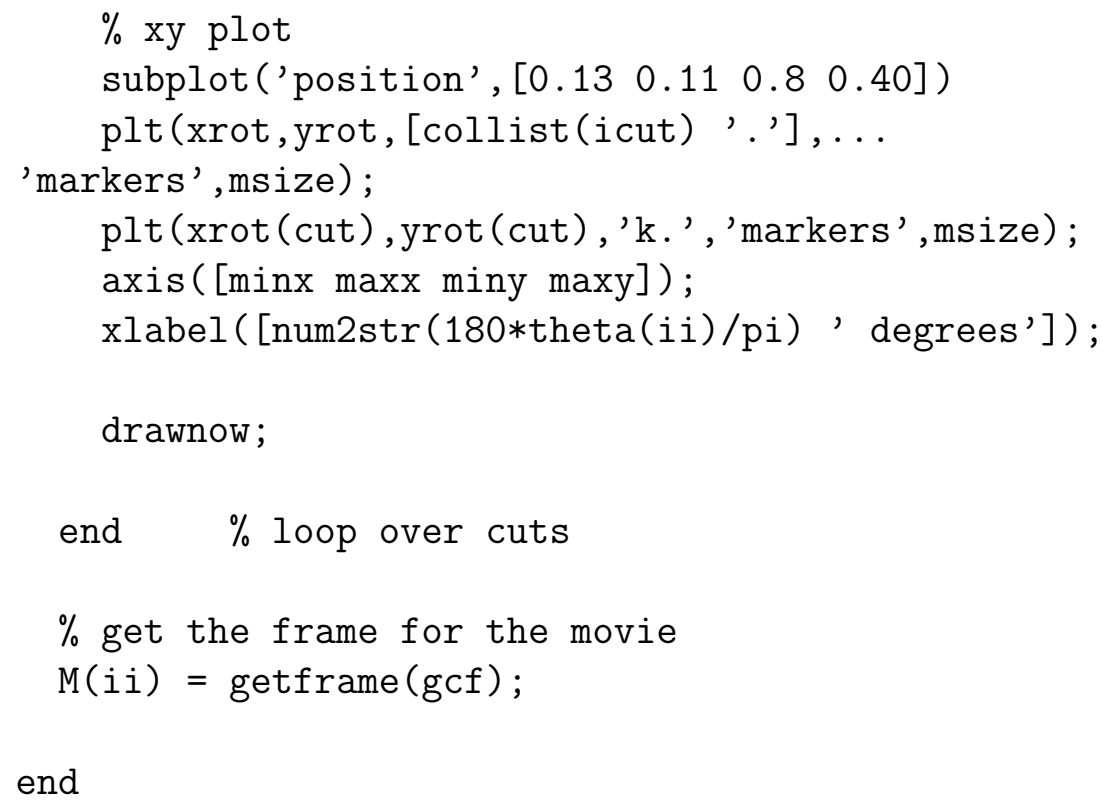

end

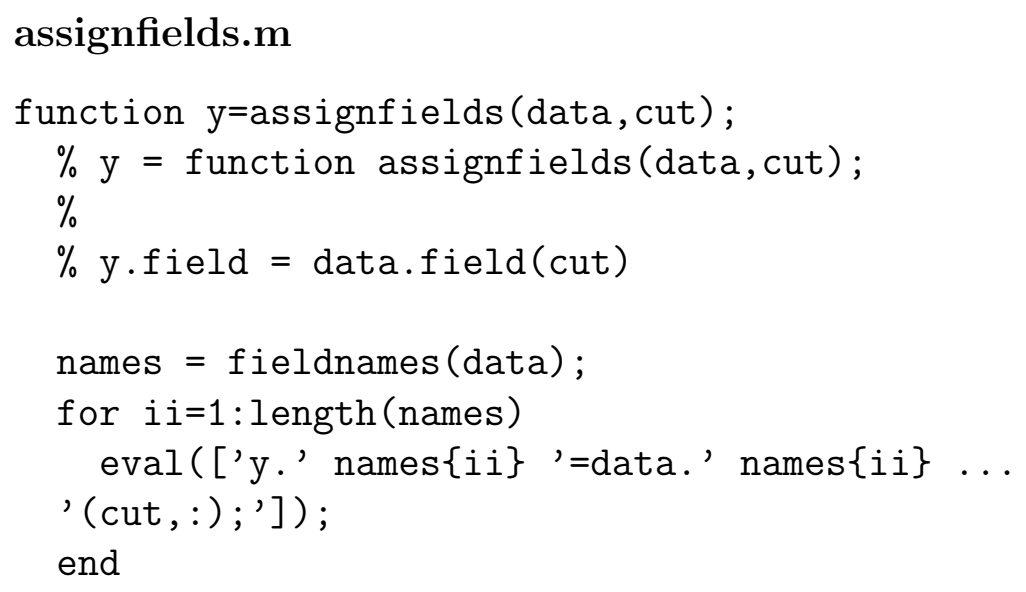


130APPENDIX A. INSTRUCTIONS AND CODE FOR MAKING LOOKUP TABLES

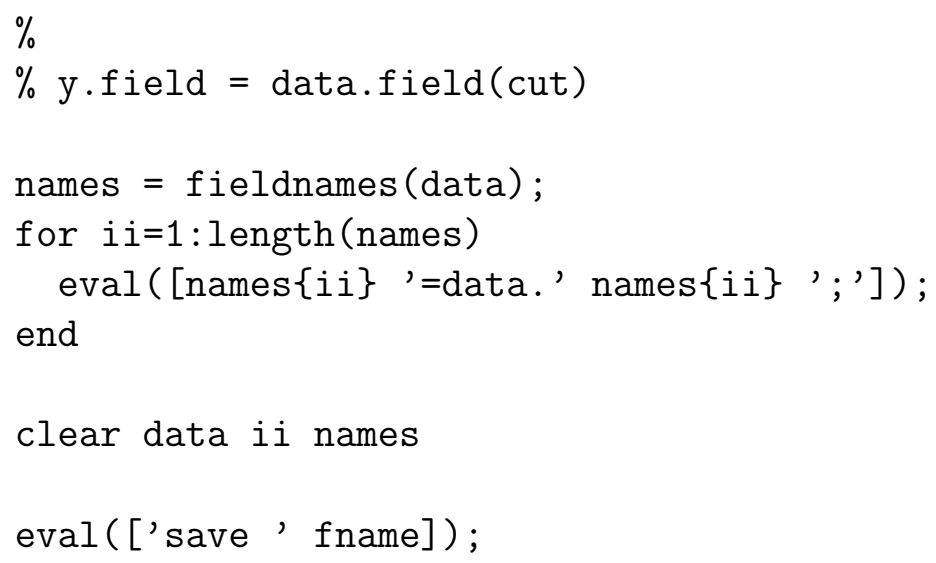




\section{Bibliography}

[1] S. Eidelman et al., Review of particle physics, Phys. Lett. B592, 1 (2004).

[2] S. M. Faber and J. S. Gallagher, Masses and mass-to-light ratios of galaxies, Ann. Rev. Astron. Astrophys. 17, 135-183 (1979).

[3] V. Trimble, Existence and nature of dark matter in the universe, Ann. Rev. Astron. Astrophys. 25, 425-472 (1987).

[4] K. G. Begeman, A. H. Broeils, and R. H. Sanders, Extended rotation curves of spiral galaxies: Dark haloes and modified dynamics, Mon. Not. Roy. Astron. Soc. 249, 523 (1991).

[5] M. Persic, P. Salucci, and F. Stel, The Universal rotation curve of spiral galaxies: 1. The Dark matter connection, Mon. Not. Roy. Astron. Soc. 281, 27 (1996).

[6] C. Alcock et al., The MACHO project: Microlensing results from 5.7 years of LMC observations, Astrophys. J. 542, 281-307 (2000).

[7] C. Renault et al., Observational Limits on Machos in the Galactic Halo, (1996).

[8] C. Alcock et al., EROS and MACHO Combined Limits on Planetary Mass Dark Matter in the Galactic Halo, (1998).

[9] D. N. Spergel et al., First Year Wilkinson Microwave Anisotropy Probe (WMAP) Observations: Determination of Cosmological Parameters, Astrophys. J. Suppl. 148, 175 (2003). 
[10] M. Tegmark et al., The 3D power spectrum of galaxies from the SDSS, Astrophys. J. 606, 702-740 (2004).

[11] M. Tegmark et al., Cosmological parameters from SDSS and WMAP, Phys. Rev. D69, 103501 (2004).

[12] G. R. Blumenthal, S. M. Faber, J. R. Primack, and M. J. Rees, Formation of galaxies and large-scale structure with cold dark matter, Nature 311, 517-525 (Oct 1984).

[13] M. Davis, G. Efstathiou, C. S. Frenk, and S. D. M. White, The evolution of large-scale structure in a universe dominated by cold dark matter, Astrophys. J. 292, 371-394 (May 1985).

[14] P. J. E. Peebles, The Gravitational-Instability Picture and the Nature of the Distribution of Galaxies, Astrophys. J.1 189, L51+ (Apr 1974).

[15] P. J. E. Peebles, Large-scale background temperature and mass fluctuations due to scale-invariant primeval perturbations, Astrophys. J.1 263, L1-L5 (Dec 1982).

[16] C. M. Baugh et al., The 2dF Galaxy Redshift Survey: Hierarchical galaxy clustering, Mon. Not. Roy. Astron. Soc. 351, L44 (2004).

[17] A. S. Szalay and J. R. Bond, Neutrino mass and galaxy formation, in IAU Symp. 104: Early Evolution of the Universe and its Present Structure, pages 307-312, 1983.

[18] Y. B. Zel'Dovich, Gravitational instability: an approximate theory for large density perturbations., Astron. Astrophys. 5, 84-89 (March 1970).

[19] B. W. Lee and S. Weinberg, Cosmological lower bound on heavy-neutrino masses, Phys. Rev. Lett. 39, 165-168 (1977).

[20] E. W. Kolb and M. S. Turner, The Early Universe, Addison-Wesley, 1989.

[21] G. L. Kane, editor, Perspectives on Supersymmetry, pages 1-98, World Scientific Publishing Company, 1998. 
[22] G. Jungman, M. Kamionkowski, and K. Griest, Supersymmetric dark matter, Phys. Rept. 267, 195-373 (1996).

[23] T. Kajita, Atmospheric neutrino results from Super-Kamiokande and Kamiokande: Evidence for nu/mu oscillations, Nucl. Phys. Proc. Suppl. 77, 123-132 (1999).

[24] Q. R. Ahmad et al., Direct evidence for neutrino flavor transformation from neutral-current interactions in the Sudbury Neutrino Observatory, Phys. Rev. Lett. 89, 011301 (2002).

[25] K. Eguchi et al., First results from KamLAND: Evidence for reactor anti- neutrino disappearance, Phys. Rev. Lett. 90, 021802 (2003).

[26] C. Athanassopoulos et al., Evidence for neutrino oscillations from muon decay at rest, Phys. Rev. C54, 2685-2708 (1996).

[27] T. Falk, K. A. Olive, and M. Srednicki, Heavy sneutrinos as dark matter, Phys. Lett. B339, 248-251 (1994).

[28] L. Covi, J. E. Kim, and L. Roszkowski, Axinos as cold dark matter, Phys. Rev. Lett. 82, 4180-4183 (1999).

[29] K. Abe et al., A measurement of the branching fraction for the inclusive B to X/s gamma decays with Belle, Phys. Lett. B511, 151-158 (2001).

[30] S. Chen et al., Branching fraction and photon energy spectrum for $b->$ s gamma, Phys. Rev. Lett. 87, 251807 (2001).

[31] G. W. Bennett et al., Measurement of the negative muon anomalous magnetic moment to 0.7-ppm, Phys. Rev. Lett. 92, 161802 (2004).

[32] Y. G. Kim, T. Nihei, L. Roszkowski, and R. Ruiz de Austri, Upper and lower limits on neutralino WIMP mass and spin- independent scattering cross section, and impact of new (g-2)(mu) measurement, JHEP 12, 034 (2002). 
[33] M. W. Goodman and E. Witten, Detectability of certain dark-matter candidates, Phys. Rev. D31, 3059 (1985).

[34] G. Bertone, D. Hooper, and J. Silk, Particle dark matter: Evidence, candidates and constraints, (2004).

[35] L. Bergstrom, Non-baryonic dark matter: Observational evidence and detection methods, Rept. Prog. Phys. 63, 793 (2000).

[36] A. Kurylov and M. Kamionkowski, Generalized analysis of weakly-interacting massive particle searches, Phys. Rev. D69, 063503 (2004).

[37] T. Saab, Search for Weakly Interacting Massive Particles with the Cryogenic Dark Matter Search Experiment, PhD thesis, Stanford University, 2002.

[38] A. Helmi, S. D. M. White, and V. Springel, The phase-space structure of a darkmatter halo: Implications for dark-matter direct detection experiments, Phys. Rev. D66, 063502 (2002).

[39] R. Bernabei et al., Search for WIMP annual modulation signature: Results from DAMA / NaI-3 and DAMA / NaI-4 and the global combined analysis, Phys. Lett. B480, 23-31 (2000).

[40] A. Benoit et al., Improved exclusion limits from the EDELWEISS WIMP search, Phys. Lett. B545, 43-49 (2002).

[41] D. Abrams et al., Exclusion limits on the WIMP nucleon cross-section from the cryogenic dark matter search, Phys. Rev. D66, 122003 (2002).

[42] D. S. Akerib et al., New results from the Cryogenic Dark Matter Search experiment, Phys. Rev. D68, 082002 (2003).

[43] S. Golwala, Exclusion Limits on the WIMP-Nucleon Elastic-Scattering Cross Section from the Cryogenic Dark Matter Search, PhD thesis, University of California, Berkeley, 2000.

[44] V. Mandic and R. Gaitskell, http://dmtools.brown.edu. 
[45] K. D. Irwin, S. W. Nam, B. Cabrera, B. Chugg, and B. A. Young, A quasiparticletrap-assisted transition-edge sensor for phonon-mediated particle detection, Review of Scientific Instruments 66(11), 5322-5326 (1995).

[46] S. Tamura, Isotope scattering of dispersive phonons in Ge, Phys. Rev. B27, 858 (1982).

[47] S. Tamura and H. J. Maris, Spontaneous decay of TA phonons, Phys. Rev. B31, 2595 (1984).

[48] H. J. Maris, Phonon propagation with isotope scattering and spontaneous anharmonic decay, Phys. Rev. B41, 9736 (1989).

[49] P. N. Luke, Voltage-assisted calorimetric ionization detector, Journal of Applied Physics 64(12), 6858-6860 (1988).

[50] B. Neganov and V. Trofimov, Otkrytia, Izobreteniya 146, 215 (1985).

[51] R. M. Clarke, P. L. Brink, B. Cabrera, P. Colling, M. B. Crisler, A. K. Davies, S. Eichblatt, R. J. Gaitskell, J. Hellmig, J. M. Martinis, S. W. Nam, T. Saab, and B. A. Young, Enhanced ballistic phonon production for surface events in cryogenic silicon detector, Applied Physics Letters 76(20), 2958-2960 (2000).

[52] K. D. Irwin, An application of electrothermal feedback for high resolution cryogenic particle detection, Applied Physics Letters 66(15), 1998-2000 (1995).

[53] K. Irwin, Phonon-Mediated Particle Detection Using Superconducting Tungsten Transition-Edge Sensors, PhD thesis, Stanford Univerisity, 1995.

[54] S. W. Nam, Development of Phonon-Mediated Cryogenic Particle Detectors with Electron and Nuclear Recoil Discrimination, PhD thesis, Stanford University, 1998.

[55] A. J. Miller, Development of a Broadband Optical Spectrophotometer Using Superconducting Transition-edge Sensors, PhD thesis, Stanford University, 2001. 
[56] R. P. Welty and J. P. Martinis, A Series Array of DC SQUIDs, IEEE Trans. Magn. 27, 2924 (1991).

[57] C. Fertig, Noise Sources in Integrated DC SQUID Series Arrays, Honors thesis, Stanford University, 1995.

[58] H. Weinstock and M. Nisenoff, editors, Superconducting Electronics, volume 59 of F: Computer and Systems Sciences, Springer-Verlag, 1988, NATO ASI Series.

[59] T. A. Shutt, A Dark Matter Detector Based on the Simultaneous Measurement of Phonons and Ionization at $20 \mathrm{mK}, \mathrm{PhD}$ thesis, University of California, Berkeley, 1993.

[60] T. Shutt et al., A solution to the dead-layer problem in ionization and phononbased dark matter detectors, Nucl. Instrum. Meth. A444, 340-344 (2000).

[61] R. Clarke, An Athermal Phonon Mediated Dark Matter Detector with Surface Event Discrimination, PhD thesis, Stanford University, 2004.

[62] R. J. Gaitskell, P. D. Barnes, A. Da Silva, B. Sadoulet, and T. Shutt, The statistics of background rejection in direct detection experiments for dark matter, Nucl. Phys. Proc. Suppl. 51B, 279-283 (1996).

[63] V. Mandic, First Results from the Cryogenic Dark Matter Search Experiment at the Deep Site, PhD thesis, University of California, Berkeley, 2004.

[64] B. A. Young, T. Saab, B. Cabrera, A. J. Miller, P. L. Brink, and J. P. Castle, Effect of implanted metal impurities on superconducting tungsten films, Journal of Applied Physics 91(10), 6516-6519 (2002).

[65] S. Kamat, Extending the Sensitivity to the Detection of WIMP Dark Matter with an Improved Understanding of the Limiting Neutron Backgrounds, PhD thesis, Case Western Reserve University, 2004.

[66] S. Yellin, Finding an upper limit in the presence of an unknown background, Physical Review D (Particles and Fields) 66(3), 032005 (2002). 
[67] E. A. Baltz and P. Gondolo, Markov chain Monte Carlo exploration of minimal supergravity with implications for dark matter, (2004), hep-ph/0407039.

[68] G. Wang, Cryogenic Dark Matter Search and Background Rejection with Event Position Information, PhD thesis, Case Western Reserve University, 2004.

[69] A. Pierce, Dark matter in the finely tuned minimal supersymmetric standard model, (2004), hep-ph/0406144. 